\title{
19th biennial IPEG Meeting
}

\author{
Nijmegen, The Netherlands. 26-30 October 2016
}

Published: 29 November 2016

\section{Training course}

\author{
A1 \\ Thalamocortical sleep oscillations \\ Igor Timofeev ${ }^{1,2}$ \\ 'Department of Psychiatry and Neuroscience, Université Laval, Québec, \\ Canada; ${ }^{2}$ Centre de recherche de l'Institut universitaire en santé mentale \\ de Québec (CRIUSMQ), Université Laval, Québec, Canada \\ Neuropsychiatric Electrophysiology 2016, 2(Suppl 1):A1
}

In waking and sleeping states, thalamocortical system generates a variety of oscillations ranging from $0.1 \mathrm{~Hz}$ to hundreds of $\mathrm{Hz}$. Most of them are present during NREM sleep, but slower activities prevail in this state of vigilance. Thalamocortical network is organized in a loop in which thalamocortical cells excite reticular thalamic and neocortical cells, reticular thalamic cells inhibit thalamocortical cells and corticothalamic cells excite thalamocortical and reticular thalamic cells. Despite stable anatomical connectivity, different types of oscillations preferentially originate either in neocortex or in thalamus. During sleep stage 2, spindle oscillation $(9-15 \mathrm{~Hz}$ ) is a dominant type of activity. It is well accepted that spindles originate in the thalamus via interplay of firing of reticular thalamic and thalamocortical neurons, but neocortex controls spindle generation. Spindles can be divided on fast and slow. Several properties of slow spindles do not match known mechanisms of their thalamic origin.

Slow oscillation (about $1 \mathrm{~Hz}$ ) dominates slow-wave sleep stage. Each slow wave is composed of hyperpolarized or silent and depolarized and active state. Active states may be accompanied by spindles and higher frequency activities. Slow waves originate mainly in deep cortical layers from which they propagate to more superficial layers and they also propagate horizontally. Full expression of slow wave activities requires the presence of thalamus, although slow oscillation can be recorded in athalamic preparations.

Therefore, despite the fact of preferential origin of different sleep oscillations in either neocortex or in thalamus, only the full thalamocortical network can generate sleep activities with known properties. Support: CIHR and NSERC.

\section{A2}

\section{Electropsychopharmacology: applying EEG and ERP to} psychopharmacology

Leon Kenemans

Experimental Psychology, Helmholtz Institute, Utrecht University, Utrecht, Netherlands

Neuropsychiatric Electrophysiology 2016, 2(Suppl 1):A2

Electroencephalography (EEG), in particular event-related or evoked potentials (ERPs), as well as their magnetic counterparts, can yield useful supplementary information when interpreting effects of psychoactive substances on behavior. They can be used to elucidate the neurocognitive mechanisms that underlie pharmacological modulation of behavior, or they may provide additional sensitivity to detect neurocognitive effects that are not readily observable in behavioral measures. This will be illustrated by means of pertinent examples. These include elucidating the mechanisms of stimulant action remediating deficient impulse control and the role of the cannabinoid system in human working memory, as well as drug effects on sensory gating and specific aspects of visual-spatial attention. Other examples concern the added sensitivity of EEG and ERP measures, relative to that of performance measures, in detecting effects of alcohol, and more generally in monitoring and predicting vigilance and the ability to detect external signals in the immediate future. Relations between brain signals and cognitive competences are revealed by either comparing different individuals, or moment-to-moment fluctuations within individuals, or differences in state (e.g., druginduced) within individuals.

A3

EEG and ERP as key techniques for functional brain alterations studies

P. F. Fabene

Department Neuroscience, Biomedicine and Movement, School of

Medicine, University of Verona, Verona, Italy

Neuropsychiatric Electrophysiology 2016, 2(Suppl 1):A3

Behavioral studies in rodents are basically based on inferring cognitive processes out of locomotor activity. In other words, we evaluate memory processes during Morris water maze, or Novel object recognition based on the time spent of the given subject in close proximity of an item, platform, or the time required to reach or leave an area of the cage/maze. By the mean of automatic scoring systems (e.g.: Ethovision, AniMaze, etc.) we are provided by objective measurements, which should be in any case interpreted by the researcher. There is always a lack of direct measurement of the cognitive processes. Integration of behavioural scoring with electrical activity evaluation of different areas involved in cognitive processing can be a useful tool, to provide scientists further parameter helpful in data interpretation. We will thus discuss the integration of EEG analysis in Alzheimer's mild cognitive impairment and acoustic ERPs in AD and epilepsy.

A4

Targeting EEG network oscillations: translational opportunities for drug discovery science in psychiatric and neurodegenerative disorders

A. Ahnaou (aahnaou@its.jnj.com)

Department of Neuroscience Discovery, Janssen Research \&

Development, a Division of Janssen Pharmaceutica NV. Turnhoutseweg 30, B-2340 Beerse, Belgium

Neuropsychiatric Electrophysiology 2016, 2(Suppl 1):A4

Despite decades of research in psychiatric and neurodegenerative disorders, the attrition rate in clinical trials and late-stage drug discovery programs for the development of novel agents for disease interception has been unacceptably high. The major issue facing neuroscience drug discovery is that drugs that show good effectivity in preclinical models often fail to meet clinical trials endpoints. The 
limitations of the traditional animal-based assays prompted a resurgence of interest in rethinking animal models and their predictability and translational validity in translational neuroscience. Better translation of a biomarker and endophenotype of the disease might rapidly provide information regarding the effects of drugs on the underlying disease biology, bridge the translational gap and potentially lower the rate of clinical trial attrition. An increasing number of experimental and clinical observations suggest that those chronic brain disorders arise from structural alterations in neuronal circuits, and therefore focus has been shifted towards investigation of electrophysiological correlates of the molecular pathology, with emphasis on neural oscillations and connectivity as promising candidate biomarkers of neuronal disorders. State-of-the-art examples of pharmacological studies modeling abnormal network oscillations and disturbed connectivity of several CNS disorders will be discussed.

A5

Source localization using LORETA software

Sebastian Olbrich (Sebastian.olbrich@puk.zh.ch)

Department for Psychiatry, Psychotherapy and Psychosomatic, University

of Zurich, Zurich, Switzerland

Neuropsychiatric Electrophysiology 2016, 2(Suppl 1):A5

The training course will be dedicated to the usage of LORETA software for localization of neuronal sources of EEG activity. After a brief introduction into the underlying physiology and theoretical assumptions of source localization techniques, an example of how to apply the software to EEG recordings will be shown, including limitations and caveats. Further focus will be on connectivity measures between intracortical areas as well as a short overview on statistical analysis implemented in the LORETA software.

A6

EEG Analysis methods beyond standard assessments

Robert Oostenveld ${ }^{1,2}$ (r.oostenveld@donders.ru.nl)

'Donders Institute for Brain, Cognition and Behaviour, Radboud

University, Nijmegen, The Netherlands; ${ }^{2}$ NatMEG, Karolinska Institute,

Stockholm, Sweden

Neuropsychiatric Electrophysiology 2016, 2(Suppl 1):A6

Data analysis methods for EEG have shown great progress over the last two decades, partially inspired or driven by methodological advancements in adjacent research methods such as MEG, intracranial recordings and functional MRI.

In this educational session I will go over the analysis methods that can be considered part of the traditional repertoire for EEG assessments and will extend that by highlighting some recent methodological advancements for data processing.

Among others, power spectral analysis techniques using multitapers, statistical approaches based on non-parametric hypothesis, cluster based inference, robust statistics and source reconstruction techniques based on spatial filtering will be explained. The focus will be on introducing these techniques in an intuitive manner and providing pointers to data analysis tools that implement them.

\section{A7}

Personalized medicine in Depression and ADHD: Introduction and EEG biomarkers for predicting treatment outcome

Martijn Arns 1,2,3 $^{1}$

'neuroCare group, Munich, Germany; ${ }^{2}$ Research Institute Brainclinics, Nijmegen, The Netherlands; ${ }^{3}$ Department of Experimental Psychology, Utrecht University, Utrecht, The Netherlands

Neuropsychiatric Electrophysiology 2016, 2(Suppl 1):A7

At present stimulant medication, antidepressants and behavior therapy are the most often applied and accepted treatments for ADHD and Depression. However, recent large-scale studies and meta-analyses have demonstrated limitations of these treatments including reduced long-term efficacy of stimulant medication, limited efficacy of antidepressant medications and overall limited efficacy of behavioral interventions on the group level. It hence becomes obvious there is a need for a re-conceptualization of psychiatric disorders along the lines of NIMH proposed Research Domain Criteria (RDoC) or referred to as biomarkers or personalized medicine. Personalized Medicine aims to prescribe the right treatment, for the right person at the right time as opposed to the currently employed 'one-sizefits-all' approach. This development relies on identification of subgroups using biomarkers.

This presentation will summarize the current status of EEG based personalized medicine and present new results from large biomarker studies in depression and ADHD focused on resting-state EEG. Several results from the iSPOT study (international Study to Predict Optimized Treatment) in Depression and ADHD will be presented $[1,2,3,4,5]$. In iSPOT-D, 1008 depressed patients are randomized to Escitalopram, Sertraline or Venlafaxine and in iSPOT-A 336 ADHD patients are prescribed with methylphenidate and patients were assessed at baseline on resting-state EEG and other measures. Several promising biomarkers that can predict treatment response and remission using baseline biomarkers will be presented and the importance of gender differences will be discussed in more detail.

\section{References}

1. Arns, M., Bruder, G., Hegerl, U., Spooner, C., Palmer, D. M., Etkin, A., . Gordon, E. (2015a). EEG alpha asymmetry as a gender-specific predictor of outcome to acute treatment with different antidepressant medications in the randomized iSPOT-D study. Clinical Neurophysiology. doi: http:// dx.doi.org/10.1016/j.clinph.2015.05.032

2. Arns, M., Etkin, A., Hegerl, U., Williams, L. M., DeBattista, C., Palmer, D. M., .Gordon, E. (2015b). Frontal and rostral anterior cingulate (rACC) theta EEG in depression: Implications for treatment outcome? European Neuropsychopharmacology,. doi: 10.1016/j.euroneuro.2015.03.007

3. Arns, M., Gordon, E., \& Boutros, N. N. (2015). EEG abnormalities are associated with poorer depressive symptom outcomes with escitalopram and venlafaxineXR, but not sertraline: Results from the multicenter randomized iSPOT-D study. Clinical EEG and Neuroscience. doi: 10.1177/1550059415621435

4. Olbrich, S., Tränkner, A., Surova, G., Gevirtz, R., Gordon, E., Hegerl, U., \& Arns, M. (2016). CNS- and ANS-arousal predict response to antidepressant medication: Findings from the randomized iSPOT-D study. Journal of Psychiatric Research, 73, 108-115. doi: 10.1016/j.jpsychires.2015.12.001

5. Olbrich, S., van Dinteren, R., \& Arns, M. (2016). Personalized medicine: Review and perspectives of promising baseline EEG biomarkers in major depressive disorder and attention deficit hyperactivity disorder. Neuropsychobiology. doi: 10.1159/000437435

A8

Clinical applications of EEG in psychiatry

Nash Boutros

University of Missouri-Kansas City, Kansas City, MO, USA

Neuropsychiatric Electrophysiology 2016, 2(Suppl 1):A8

Starting with an outline of the evolving discipline of Clinical Psychiatric Electrophysiology a discussion of the value of diagnostic tests in general is given. The presentation then focuses on the well-established clinical indications of the standard EEG in the day to day practice of clinical psychiatry. Discussion will cover panic disorder, autistic spectrum disorders, and repeated aggression in some detail. Case vignettes are included to generate interactive discussion.

\section{Keynotes}

A9

The genesis of EEG phenomena: hot topics of the last decade

Fernando Lopes da Silva (f.h.lopesdasilva@uva.nl) Centre of Neuroscience, Swammerdam Institute for Life Sciences, University of Amsterdam, Science Park 904, Amsterdam 1098 XH, The Netherlands

Neuropsychiatric Electrophysiology 2016, 2(Suppl 1):A9 
Since the turn of the century, the scope of EEG investigations became much broader, in particular due to the possibility of recording the full, physiologically relevant range of brain activities from the infra-slow to the high frequency spectral range, making use of wide dynamic direct-current (DC) coupled amplifiers, and of accurate recordings of high frequency oscillations up to hundreds of $\mathrm{Hz}$. This has been denominated full-band or wide-band EEG. In this lecture, however, I focus on the high frequency EEG/MEG phenomena or High Frequency Oscillations (HFOs). These phenomena cover a number of activities that range from $60-80 \mathrm{~Hz}$ to approximately $500 \mathrm{~Hz}$. Interest for these phenomena has gained momentum in the last decade. They appear in the healthy brain associated with sensory, motor and cognitive events, and also in pathological cases, particularly in epilepsy. Under the concept HFO, activities in the gamma band $(30-70 \mathrm{~Hz})$ occupy a prominent place. A variety of names are used to describe physiological EEG/MEG activity above $70 \mathrm{~Hz}$ such as high-gamma and the chi-band; in the context of epilepsy specific types of oscillations have been described, namely ripples $(\approx 90-200 \mathrm{~Hz})$ and fast ripples $(\approx>200 \mathrm{~Hz})$, but the corresponding frequency boundaries are rather fuzzy [1]. Here I will discuss neuronal processes generating HFOs [1,2], a few relevant applications in cognitive neuroscience and in epilepsy [3], and practical questions regarding the possibilities and difficulties of reliably recording HFOs in human, and distinguishing the latter from artifacts [4].

\section{References}

1. Buzsáki, G. and Lopes da Silva, F.H., High frequency oscillations in the intact brain. Progr. Neurobiol. 2012; 98: 241-249.

2. Jefferys, JG, Menendez de la Prida, L, Wendling, F, Bragin, A, Avoli, M, Timofeev, I, Lopes da Silva, FH. Mechanisms of physiological and epileptic HFO generation. Progr Neurobiol. 2012; 98: 250-264.

3. Lopes da Silva, F.H., EEG and MEG: relevance to Neuroscience. Neuron, 2013; 80: $1112-1128$.

4. Amiri, M, Lina, J-M, Pizzo, F, Gotman, J. High frequency oscillations and spikes: separating real HFOs from false oscillations. Clin. Neurophysiol. 2016; 127: 187-196.

\section{A10}

Cellular basis of sleep slow oscillation: What is clear, what is not Igor Timofeev ${ }^{1,2}$

'Department of Psychiatry and Neuroscience, Université Laval, Québec, Canada; ${ }^{2}$ Centre de recherche de l'Institut universitaire en santé mentale de Québec (CRIUSMQ), Université Laval, Québec, Canada

Neuropsychiatric Electrophysiology 2016, 2(Suppl 1):A10

The major type of activity generated by the thalamocortical system during slow-wave sleep is the slow oscillation, composed of slow waves repeated with a frequency of about $1 \mathrm{~Hz}$. Each slow wave is comprised of hyperpolarized silent state, often called down state, and depolarized active state, often called up state. During wake and REM sleep cortical neurons remain in active state.

What is likely known on slow wave generation: (a) Slow oscillation is essentially cortical in origin, but it is modulated by thalamic activities. (b) Cortical slow waves in adults start more in frontal areas and propagate to other cortical areas, but multiple slow waves recorded throughout cortical mantle remain local. (c) In ferrets, mice and cats slow waves start mainly in layer 5, but in epileptic patients they originate around layer 3. (d) Silent states of slow oscillation are essentially periods of disfacilitation, but GABAergic activities can be detected in a subset of neurons prior to the onset of silent states. (e) Active states are dominated by excitatory and inhibitory synaptic activities; in anesthetized animals, these activities are balanced, in sleeping animals inhibition largely dominates active states.

What we don't know: (a) What triggers the onset of active state? Three hypotheses are present: (1) Stochastic summation of spikeindependent minis occasionally leading to the first spike that engages the whole network. (2) Intrinsic activity of layer 5 pyramidal cells (h-like current). (3) Self-organized onset of activity in groups of neurons. (b) What terminates active states? Following hypotheses are proposed: (1) Intrinsic neuronal firing frequency accommodation that decreases the overall excitatory drive. (2) Use-dependent synaptic depression. (3) Activity of $\mathrm{Na}^{+}-$and $\mathrm{Ca}^{2+}$-dependent $\mathrm{K}^{+}$current. (4) Extracortical signaling. All these mechanisms are present during wake and REM sleep, but why active states are not terminated in these states of vigilance remains unclear.

Support: CIHR and NSERC.

\section{A11}

GABAergic modulation of neuronal oscillations in animals and humans and its consequences for working memory

Ole Jensen (o.jensen@bham.ac.uk)

School of Psychology, University of Birmingham, Birmingham, B15 2TT, UK

Neuropsychiatric Electrophysiology 2016, 2(Suppl 1):A11

\section{Background}

Networks in the brain must rely on powerful mechanism for routing and prioritizing information processing. In a larger set of attention and memory studies we have investigated the notion that alpha oscillations $(9-12 \mathrm{~Hz})$ are inhibitory and serve to route the information flow: 'gating by inhibition' [1]. The alpha band activity is under top-down control by areas in the dorsal attention network. As such the alpha band activity - previously believed to reflect a state of rest - serves an important role for shaping the functional architecture of the working brain. Gamma band activity $(50-100 \mathrm{~Hz})$ reflects feed-forward processing and is modulated by the alpha oscillations. In animals is has been demonstrated that GABAergic interneurons play an important role for synchronizing neural populations [2]; however, it remains unknown if these mechanistic principles generalize to human oscillations.

Methods

To investigate how GABAergic modulated affects gamma oscillations, we recorded ongoing brain activity using magnetoencephalography (MEG) in human subjects participating in a double-blind pharmacological study receiving placebo and lorazepam. Lorazepam is a benzodiazepine upregulating GABAergic conductance. This was done in participants while they performed a visuospatial working memory (WM) task.

Results

The key finding was that occipital gamma power associated with WM recognition increased with lorazepam dosage [3]. In addition, the frequency of the gamma activity decreased with dosage. This is consistent with models derived from the rat hippocampus. With respect to oscillations in the alpha band, we observed a parametrical decrease with drug dosage that also predicted a performance decrease. This is consistent with alpha oscillations reflecting functional inhibition.

\section{Conclusion}

We conclude that GABAergic interneurons are implicated in the generation of gamma and alpha oscillations in humans. As we will discuss these findings allow us to link neuronal dynamics to behavior in humans by embracing established animal models.

\section{References}

1. Jensen $O$ and Mazaheri A. Shaping functional architecture by oscillatory alpha activity: gating by inhibition. Front Hum Neurosci 2010; 4:186.

2. Traub RD, Jefferts JGR, Whittington MA. Fast Oscillations in Cortical Circuits. Boston: MIT Press, 1999

3. Lozano-Soldevilla D, ter Huurne N, Cools R, Jensen O. GABAergic modulation of visual gamma and alpha oscillations and its consequences for working memory performance. Curr Biol 2014; 24:2878-2887.

\section{A12}

Refining brain oscillatory targets for intervention in ADHD Sandra K Loo

Department of Psychiatry and Biobehavioral Sciences, UCLA, Los Angeles, CA, USA

Neuropsychiatric Electrophysiology 2016, 2(Suppl 1):A12

\section{Background}

Brain oscillatory patterns are increasingly used as biological markers of psychiatric disease, developmental course, and treatment response. In 
order to maximize effectiveness, advanced approaches should enable a mechanistic understanding of how brain rhythms carry out the cognitive and emotional processes that, when disordered, may lead to mental disorders. This will aide in the identification of interventions that show evidence of mechanism and target engagement and will increase our understanding of how efficacious interventions achieve their effect within clinical populations

In this presentation, important considerations in the process of identifying and refining potential brain oscillatory targets that may be useful for treatment monitoring and response will be presented. For example, approaches to characterizing the significant variability and heterogeneity that exists within ADHD and typically developing populations are discussed. In addition, the need for refined measurements and signal processing techniques that increase the signal to noise ratio are described. Finally, issues such as targeting cognitive dysfunction and/or modeling developmental changes are considered.

We then describe how this approach has been implemented to identify and validate brain targets (biomarkers) in clinical trials for children with attention-deficit/hyperactivity disorder (ADHD). In addition, these data will be used to illustrate potential applications for neuromodulation approaches in ADHD as well as other neurodevelopmental disorders such as autism spectrum disorder (ASD) and Tourette's Syndrome.

\section{Relevance and Implications for future research}

The data presented suggest that EEG-based biomarkers may be useful indices of developmental course of disorder, behavioral and cognitive functioning, and prediction of treatment response. Although the clinical utility of EEG measures is promising, more research is needed before these findings can be implemented in clinical practice.

\section{A13}

Elucidating mechanisms of sleep-wake regulation in humans with pharmaco-genetic tools

Hans-Peter Landolt 1,2

${ }^{1}$ Institute of Pharmacology and Toxicology, University of Zürich, Zürich, Switzerland; ' Zürich Center for interdisciplinary Sleep Research,

University of Zürich, Zürich, Switzerland

Neuropsychiatric Electrophysiology 2016, 2(Suppl 1):A13

Epidemiological studies demonstrate that sleep-wake disorders are highly prevalent in society and rank third in the prevalence of all brain diseases. The normal alternation between sleep and wakefulness is tightly regulated, and prolonged EEG recordings show that distinct sleep and wake states reflect highly complex behaviors. Little is currently known about the molecular underpinnings of physiological sleep-wake regulation and functions. To foster our knowledge of the pathophysiology of sleep-wake disorders and their possible rational treatment, a molecular understanding of sleep-wake regulatory processes is indispensable. Accumulating evidence suggests that important aspects of sleep-wake regulation in animals and humans are genetically controlled and, thus, have a molecular basis. Consistent with this view, the combination of neurophysiologic, genetic and pharmacologic tools revealed specific roles for adenosine, dopamine and glutamate receptors and metabolic pathways in sleep-wake regulation. These studies also showed that functional allelic variation in candidate genes can profoundly affect functional aspects of sleep and wakefulness, even in healthy humans and under physiological conditions, as well as modulate individual responses to hypnotic and wake-promoting agents. These insights may provide a rationale for personalized sleep-wake pharmacotherapy (Holst et al., Annu Rev Phamacol Toxicol, 2016). In the future, together with novel 'omics'-studies of sleep in health and disease, they may pave the way for the discovery of new evidence-based treatments of sleep-wake pathologies such as insomnia and the pharmacological enhancement of sleepassociated brain functions such as neuronal plasticity.

Research supported by the Swiss National Science Foundation (grant \# 320030135414 \& 320030 163439) and the Clinical Research Priority Program "Sleep and Health" of the University of Zürich.

\section{Symposia}

\section{A14}

The dos and don'ts for electrophysiological connectivity analysis

Jan Mathijs Schoffelen (j.schoffelen@donders.ru.nl)

Donders Institute, Radboud University, Nijmegen, The Netherlands

Neuropsychiatric Electrophysiology 2016, 2(Suppl 1):A14

In recent years it has been increasingly recognized that insight into the dynamics of interareal interactions is crucial for our understanding of normal and pathological brain function. Methodological developments and open source availability of advanced analysis tools have enabled the wider neuroscientific community to estimate a wide range of connectivity metrics from noninvasively obtained electrophysiological signals. Next to deciding on an appropriate analysis strategy, researchers are faced with the challenge to correctly interpret their findings. Volume conduction and electromagnetic field spread cause neuronal signals to be picked up by multiple channels at once, causing spurious estimates of connectivity. Comparison across experimental groups and conditions may be confounded by differences in univariate signal properties such as signal-to-noise ratio. I will illustrate some of these interpretational pitfalls and provide some recommendations that may need to be taken into account to improve the validity of the interpretation of EEG/MEG connectivity studies.

\section{A15}

MEG as a routine diagnostic tool in memory clinic patients

Alida A. Gouww ${ }^{1,2}$, Arjan Hillebrand ${ }^{2}$, Matteo Demuru ${ }^{1,2}$, Peterjan Ris ${ }^{2}$,

Philip Scheltens ${ }^{1}$, Cornelis J Stam²

${ }^{1}$ Alzheimer Center and Department of Neurology, Neuroscience Campus Amsterdam, VU University Medical Center, Amsterdam, The Netherlands; ${ }^{2}$ Department of Clinical Neurophysiology and MEG 4Center,

Neuroscience Campus Amsterdam, VU University Medical Center, Amsterdam, The Netherlands

Correspondence: Alida A Gouw (aa.gouw@vumc.nl)

Neuropsychiatric Electrophysiology 2016, 2(Suppl 1):A15

\section{Background}

Electro-encephalography (EEG) has been used as a routine diagnostic tool for patients of the Alzheimer Center Amsterdam since 2001 [1]. Recently, EEG has partly been replaced by magneto-encephalography (MEG), because it may be more sensitive for pathology in disease specific regions. We investigated its discriminative value between Alzheimer's disease (AD) and subjective cognitive decline (SCD) using a machine learning approach.

Methods

MEG was recorded in an unselected proportion of memory clinic patients as part of a routine workup. MEG data were co-registered with a head-size matched template-MRI and source-reconstructed by projection onto 90 AAL-regions using beamforming [2, 3]. Clinical reports were made using visual and spectral analyses, blinded for clinical information. Diagnoses were made in a weekly multidisciplinary meeting using full clinical information and additional investigations, such as MRI and neuropsychological examination. The first $20 \mathrm{AD}$ and $20 \mathrm{SCD}$ patients were further analysed. Directed connectivity (directed phase transfer entropy [dPTE]) and minimum spanning tree (MST) based network measures $(8-13 \mathrm{~Hz}$ band) were calculated per region [3-5], where the imbalance in information flow between regions was used to construct the MST. Combinations of MEG measures at eight AD-specific regions (left and right hippocampus, parahippocampal gyrus, precuneus, cuneus) were entered into random forest models to classify between patient groups.

Results

From April 2015 to July 2016, 101 patients received an MEG. Diagnoses were AD ( $n=26)$; SCD $(n=24)$; psychiatric disorder $(n=18)$; mild cognitive impairment $(n=10)$; fronto-temporal dementia $(n=7)$; Lewy body dementia $(n=5)$; vascular dementia $(n=1)$; and other/ 
postponed diagnosis $(n=10)$. One patient's MEG diagnostic report could not be made because of movement artefacts. Her MRI and neuropsychological examination could also not be completed and she was diagnosed with severe AD based on clinical information and cerebrospinal fluid biomarkers. In the distinction between AD (age $64.8 \pm 7.9,50 \%$ female) and SCD (age $61.4 \pm 21.8,55 \%$ female), a random forest model with relative theta power of the eight $A D$ regions yielded an accuracy of 0.810 . Addition of dPTE for these regions increased the accuracy to 0.843 . When network measures (leaf fraction, diameter, tree hierarchy) were added to the model with theta power and dPTE an accuracy of 0.812 was found.

\section{Conclusion}

Routine diagnostic MEG is feasible in a memory clinic screening and has a high accuracy in the discrimination between $A D$ and SCD using theta power in AD-specific regions. Directed connectivity has modest additional diagnostic value whereas network measures did not add to the diagnostic accuracy.

\section{References}

1. Liedorp M, van der Flier WM, Hoogervorst EL, Scheltens P, Stam CJ. Associations between patterns of EEG abnormalities and diagnosis in a large memory clinic cohort. Dement Geriatr Cogn Disord 2009; 27(1):18-23.

2. Hillebrand A, Barnes GR, Bosboom JL, Berendse HW, Stam CJ. Frequencydependent functional connectivity within resting-state networks: an atlas-based MEG beamformer solution. Neuroimage 2012; 59(4):3909-21.

3. Hillebrand A, Tewarie P, van Dellen E, Yu M, Carbo EWS, Douw L, et al. Direction of information flow in large-scale resting-state networks is frequency-dependent. PNAS 2016; 113(14):3867-72.

4. Stam CJ, Tewarie P, Van Dellen E, van Straaten ECW, Hillebrand A, Van Mieghem P. The trees and the forest: Characterization of complex brain networks with minimum spanning trees. Int J Psychophysiol 2014; 92(3):129-38.

5. Lobier M, Siebenhühner F, Palva S, Palva JM. Phase transfer entropy: a novel phase-based measure for directed connectivity in networks coupled by oscillatory interactions. Neuroimage 2014; 85:853-72.

\section{A16}

\section{Identification of the epileptogenic zone using MEG network} analysis

Ida A. Nissen ${ }^{1}$, Cornelis J. Stam', Ilse E. C. W. van Straaten', Jaap C. Reijneveld ${ }^{2}$, Arjan Hillebrand ${ }^{1}$

${ }^{1}$ Department of Clinical Neurophysiology and MEG Center, VU University Medical Center, Amsterdam, the Netherlands; ${ }^{2}$ Brain Tumor Center Amsterdam \& Department of Neurology, VU University Medical Center, Amsterdam, the Netherlands

Correspondence: Ida A. Nissen (i.nissen@vumc.nl)

Neuropsychiatric Electrophysiology 2016, 2(Suppl 1):A16

\section{Introduction}

Epilepsy is increasingly seen as a brain network disorder [1-3]. Patients with epilepsy have been shown to have different networks compared to healthy controls, deviating from the optimal configuration and with abnormal network hubs [4-8]. A potent treatment for pharmacoresistant epilepsy is epilepsy surgery. The goal of epilepsy surgery is to remove or disconnect the epileptogenic zone, which renders the patient seizurefree [9]. A hypothesis about the location of the epileptogenic zone can be based on techniques such as electroencephalography (EEG) and magnetoencephalography (MEG). However, establishing a hypothesis is challenging and not always successful as currently one third of the patients continue to experience seizures after surgery [10]. New methods are therefore needed to generate more accurate hypotheses about the location of the epileptogenic zone such that more patients become seizure-free. Our aim was to develop such a new method based on network theory. We hypothesized that the epileptogenic zone coincides or connects with hubs and information senders in the network.

\section{Methods}

We analyzed eyes-closed resting-state MEG recordings of 22 patients with pharmacoresistant epilepsy. The time series in source space (virtual electrodes) were reconstructed using beamforming for 90 regions of the AAL atlas [11]. We estimated functional connectivity between those regions using phase lag index (PLI) [12] in the broadband $(0.5-48 \mathrm{~Hz})$. We used 20 epochs of $3.28 \mathrm{~s}$ each without artefacts or epileptiform activity. We generated the minimum spanning tree based on the PLI and calculated the betweenness centrality (an indicator of hubs) for each region. Furthermore, we assessed effective connectivity (an indicator of information senders) using the directed phase transfer entropy (dPTE) [11] for different frequency bands. Results

ROIs with high broadband betweenness centrality (hubs) coincided with the resection cavity (or resection lobe) in $8 / 14$ (9/14) seizurefree patients and in $0 / 8(0 / 8)$ patients with remaining seizures $(73 \%$ (77 \%) accuracy). For the effective connectivity, high dPTE values coincided with the resection cavity (or resection lobe) in 8/14 (10/14) seizure-free patients and only in $2 / 8(2 / 8)$ patients with remaining seizures $(64 \%(73 \%)$ accuracy) in the delta band $(0.5-4 \mathrm{~Hz})$.

Implications

Hub regions and strong senders are markers of the epileptogenic zone. These results are a first step towards a localization method that can be applied to MEG recordings even in the absence of epileptiform activity, yielding an improvement in localization and finally surgery outcome.

\section{References}

1. Kramer MA, Cash SS: Epilepsy as a disorder of cortical network organization. The neuroscientist 2012, 18: 360-372.

2. Stam CJ: Modern network science of neurological disorders. Nature Reviews Neuroscience 2014, 15: 683-695.

3. van Diessen E, Diederen SJ, Braun KP, Jansen FE, Stam CJ: Functional and structural brain networks in epilepsy: what have we learned? Epilepsia 2013, 54: 1855-1865.

4. Bernhardt BC, Chen Z, He Y, Evans AC, Bernasconi N: Graph-theoretical analysis reveals disrupted small-world organization of cortical thickness correlation networks in temporal lobe epilepsy. Cerebral cortex 2011, 21: 2147-2157.

5. Jin S-H, Jeong W, Chung CK: Mesial temporal lobe epilepsy with hippocampal sclerosis is a network disorder with altered cortical hubs. Epilepsia 2015, 56: 772-779.

6. Liao W, Zhang Z, Pan Z, Mantini D, Ding J, Duan X et al.: Altered functional connectivity and small-world in mesial temporal lobe epilepsy. Plos one 2010, 5: e8525.

7. van Diessen E, Hanemaaijer Jl, Otte WM, Zelmann R, Jacobs J, Jansen FE et al.: Are high frequency oscillations associated with altered network topology in partial epilepsy? Neuroimage 2013, 82: 564-573.

8. Zhang Z, Liao W, Chen H, Mantini D, Ding JR, Xu Q et al.: Altered functional-structural coupling of large-scale brain networks in idiopathic generalized epilepsy. Brain 2011, 134: 2912-2928.

9. Luders HO, Najm I, Nair D, Widdess-Walsh P, Bingman W: The epileptogenic zone: general principles. Epileptic disorders 2006, 8: S1.

10. Jobst BC, Cascino GD: Resective Epilepsy Surgery for Drug-Resistant Focal Epilepsy: A Review. JAMA 2015, 313: 285-293.

11. Hillebrand A, Tewarie P, van Dellen E, Yu M, Carbo EW, Douw L et al.: Direction of information flow in large-scale resting-state networks is frequency-dependent. Proceedings of the National Academy of Sciences 2016, 113: 3867-3872.

12. Stam CJ, Nolte G, Daffertshofer A: Phase lag index: assessment of functiona connectivity from multi-channel EEG and MEG with diminished bias from common sources. Human brain mapping 2007, 28: 1178-1193.

\section{A17}

EEG biomarker integration for better decision making in clinical

trials

Sonja Simpraga', Ricardo Alvarez-Jimenez², Huibert D Mansvelder', Joop MA van Gerven ${ }^{2}$, Geert Jan Groeneveld ${ }^{2,3}$, Simon-Shlomo Poil ${ }^{1,4^{*}}$, Klaus Linkenkaer-Hansen ${ }^{1 *}$

'Dept. Integrative Neurophysiology, CNCR, Neuroscience Campus Amsterdam, Vrije Universiteit Amsterdam, Amsterdam, Netherlands; ${ }^{2}$ Dept. Clinical Pharmacology, Centre for Human Drug Research, Leiden, Netherlands; ${ }^{3}$ Dept. Neurology, VU University Medical Center, Amsterdam, Netherlands; ${ }^{4}$ NBT Analytics BV, Amsterdam, Netherlands Neuropsychiatric Electrophysiology 2016, 2(Suppl 1):A17

*These authors contributed equally 
Brain disorders are a huge burden on the health care system, key issues being inaccurate diagnosis and insufficient treatment options. Hence, there is an urgent need for biomarkers that monitor disease status or therapeutic response. Current biomarkers lack the desired accuracy, because of the large variability in healthy subjects and the often subtle disease-related changes. In EEG, however, pathophysiology is often expressed in multiple ways. Here we show that an integrative approach in which any biomarker that carries complementary information about a disease or therapeutic intervention can result in an accurate diagnostic index for better decision making in clinical trials.

Recently, we showed that EEG biomarker integration improves the prediction of conversion from mild cognitive impairment to Alzheimer's disease (AD) compared with a single-biomarker based prediction [1]. The integrative biomarker index can be used for stratification of patients at recruitment in clinical studies and for documenting and quantifying effects of intervention.

Here, we provide additional proof-of-concept that EEG-based prediction can be improved with the integrative biomarker approach in clinical trials where a drug is tested in a scopolamine challenge model in healthy subjects. Scopolamine is the most extensively studied and used model for cognitive impairment and resembles the changes seen in $A D$ patients [2]. It is used in drug development to demonstrate the reversal of the temporary scopolamine-induced cognitive deficits by a cognition enhancing compound. For this purpose, we have developed an integrative EEG-biomarker index ( $\mathrm{mAChR}$ index) that is optimally sensitive to the CNS effects of scopolamine, to objectively determine whether reversal of scopolamine effects by a cholinergic compound is successful. The mAChR index yielded higher classification performance than any individual EEG biomarker with accuracy, sensitivity, specificity and precision of $90 \%$. This significantly outperforms the single-best EEG biomarker (relative delta power). Validation on an independent dataset indicated the robustness of the index. To examine the validity of scopolamine as a cognitive impairment model, we applied this integrative index on healthy elderly controls and Alzheimer's patients and observed that this index indeed differentiates patients from controls.

We address this by using novel features of the Neurophysiological Biomarker Toolbox (http://www.nbtwiki.net/), which employ datamining algorithms to combine the information from multiple biomarkers. Our results demonstrate that integrating information from multiple EEG biomarkers better captures the unique phenotype of an individual patient and is a promising approach to enhance accuracy and reduce the multiple-comparisons problem when using EEG in clinical trials.

\section{References}

1. Poil SS, de Haan W, van der Flier WM, Mansvelder HD, Scheltens $P$, Linkenkaer-Hansen K. Integrative EEG biomarkers predict progression to Alzheimer's disease at the MCl stage. Front Aging Neurosci. 2013; 5:58

2. Klinkenberg I, Blokland A. The validity of scopolamine as a pharmacological model for cognitive impairment: A review of animal behavioral studies. Neurosci Biobehav Rev. 2010; 34:1307-50.

\section{A18}

\section{Continuous EEG and deep learning for outcome prediction in} postanoxic coma

Michel JAM van Putten ${ }^{1,2}$, Marleen C Tjepkema-Cloostermans ${ }^{1}$, Jeannette Hofmeijer 2,3

'Department of neurology and clinical neurophysiology, Medisch Spectrum Twente, Enschede, Netherlands; ${ }^{2}$ Clinical Neurophysiology, University of Twente, Enschede, the Netherlands; ${ }^{3}$ Department of neurology, Rijnstate Ziekenhuis, Arnhem, Netherlands

Correspondence: Michel JAM van Putten

(m.j.a.m.vanputten@utwente.nl)

Neuropsychiatric Electrophysiology 2016, 2(Suppl 1):A18

\section{Introduction}

Reliable outcome prediction in approximately $50 \%$ of patients with a postanoxic encephalopathy is possible with visual interpretation of continuous EEG recorded within $24 \mathrm{~h}$ after cardiac arrest [1-6]. To assist in the visual assessment, we developed the Cerebral Recovery Index [7] and, more recently, a random forest classifier, showing similar performance for outcome prediction as visual assessment of the EEG [8]. Deep Learning may advance the prognostic value of EEG significantly, in part as it does not depend on 'hand-made' features $[3,9]$.

Methods

We used data from the EEG database of the Medisch Spectrum Twente and Rijnstate hospitals with recordings from patients treated in the Intensive Care Unit with a postanoxic encephalopathy after a cardiac arrest. EEGs were recorded with twenty-one silver/silver chloride cup electrodes placed on the scalp according to the international 10-20 system using a Neurocenter EEG recording system (Clinical Science Systems, Voorschoten, The Netherlands) or a Nihon Kohden system (VCM Medical, the Netherlands). Neurological outcome (Cerebral performance category scores) was dichotomized as good (no or mild neurological impairment) or poor (severe neurological impairment, vegetative state or death) at 6 months after cardiac arrest.

We implemented a convolutional neural network (CNN) in python with TensorFlow on a CentOS system with the NVIDIA GTX-1080 as GPU. The input layer had dimensions $128 \times 19$ to process the raw 19channel EEG. EEGs were analyzed using non-overlapping $2 \mathrm{~s}$ epochs using 5 min segments at each hour after cardiac arrest. For each patient in the validation set, we calculated the percentage of $2 \mathrm{~s}$ epochs within the $5 \mathrm{~min}$ segment that is predicted as poor neurological outcome. Using ROC curves the threshold at which poor outcome could be predicted with $100 \%$ specificity was determined.

Results

After training with $131 \mathrm{EEGs}$, evaluation in an independent set with 33 patients showed that poor outcome could reliably be predicted in $67 \%$ of the patients, without false positives (specificity $100 \%$ ) at = $12 \mathrm{~h}$ after cardiac arrest; poor outcome prediction at a later instance $(t=24)$ was not possible with a specificity of $100 \%$.

Discussion and Conclusion

We show feasibility of CNN to process EEG in patients with a postanoxic coma for prognostication. Pilot results show high predictive value for poor neurological outcome. As temporal evolution of EEG patterns in these patients is significant [2], recurrent neural nets may outperform convolutional networks. To understand the discriminating features, we currently explore methods for interpretation and visualization of networks.

\section{References}

1. Hofmeijer, J., T. M. J. Beernink, F. H. Bosch, A. Beishuizen, M. C. TjepkemaCloostermans and M.J.A.M. van Putten. "Early EEG contributes to multimodal outcome prediction of postanoxic coma." Neurology 2015; (85): 1-7.

2. Hofmeijer, J. and MJ.A.M. Van Putten (2016). "EEG in postanoxic coma: prognostic and diagnostic value." Clinical Neurophysiology 127(4): 2047-2055.

3. LeCun, Y., Y. Bengio and G. Hinton. "Deep learning." Nature 2015; 13(1): 35-35.

4. Rossetti, A. O., A. A. Rabinstein and M. Oddo. "Neurological prognostication of outcome in patients in coma after cardiac arrest." The Lancet Neurology 2016; 15(6): 597-609

5. Sivaraju, A., E. J. Gilmore, C. R. Wira, A. Stevens, N. Rampal, J. J. Moeller, D. M. Greer, L. J. Hirsch and N. Gaspard. "Prognostication of post-cardiac arrest coma: early clinical and electroencephalographic predictors of outcome." Intensive Care Medicine 2015; 41(7): 1264-1272.

6. Tjepkema-Cloostermans, M. C., J. Hofmeijer, R. J. Trof, M. J. Blans, A. Beishuizen and M.JA.M. van Putten. "Electroencephalogram predicts outcome in patients with postanoxic coma during mild therapeutic hypothermia." Critical care medicine 2015; 43(1).

7. Tjepkema-Cloostermans, M. C., F. B. van Meulen, G. Meinsma and M.J.A.M. van Putten. "A Cerebral Recovery Index (CRI) for early prognosis in patients after cardiac arrest." Critical Care 2013; 17(5): R252-R252.

8. Tjepkema-Cloostermans, M. C., J. Hofmeijer, A. Beishuizen, H. Hom, M. J. Blans, F.H. Bosch and M.J.A.M. van Putten. "Cerebral Recovery Index: reliable help for prediction of neurological outcome after cardiac arrest". Submitted 2016

9. Angermueller, C., T. Pärnamaa, L. Parts and S. Oliver. "Deep Learning for Computational Biology." Molecular Systems Biology, 2016; (12): 878-878. 
A19

Prospects and challenges of Alzheimer's classification using resting-state EEG rhythms

Claudio Babiloni ${ }^{1,2}$, Antonio Ivano Triggiani ${ }^{3}$, Roberta Lizio ${ }^{1,2}$, Susanna Cordone ${ }^{1}$, Antonio Brunetti ${ }^{4}$, Giacomo Tattoli ${ }^{4}$, Vitoantonio Bevilacqua ${ }^{4}$, Andrea Soricelli ${ }^{5,6}$, Raffaele Ferri ${ }^{7}$, Flavio Nobili ${ }^{8}$, Loreto Gesualdo9 ${ }^{9}$, José Carlos Millán-Calenti ${ }^{10}$, Ana Buján ${ }^{10}$, Rosanna Tortelli ${ }^{11}$, Valentina Cardinali ${ }^{12,16}$, Orietta Barulli ${ }^{11}$, Antonio Giannini ${ }^{13}$, Pantaleo Spagnolo ${ }^{14}$, Silvia Armenise ${ }^{15}$, Grazia Buenza ${ }^{12}$, Gaetano Scianatico ${ }^{11}$, Giancarlo Logroscino $^{11,15}$, Giovanni B. Frisoni ${ }^{16,17,18}$, and Claudio Del Percio ${ }^{5}$ 'Department of Physiology and Pharmacology "Vittorio Erspamer", University of Rome "La Sapienza", Rome, Italy; ${ }^{2}$ Department of Neuroscience, IRCCS San Raffaele Pisana, Rome, Italy; ${ }^{3}$ Department of Clinical and Experimental Medicine, University of Foggia, Foggia, Italy; ${ }^{4}$ Department of Electrical and Information Engineering, Polytechnic of Bari, Bari, Italy; ${ }^{5}$ Department of Integrated Imaging, IRCCS SDN, Napoli, Italy; ${ }^{6}$ Department of Motor Sciences and Healthiness, University of Naples Parthenope, Naples, Italy; ${ }^{7}$ Department of Neurology, IRCCS Oas Institute for Research on Mental Retardation and Brain Aging, Troina, Enna, Italy; ${ }^{8}$ Service of Clinical Neurophysiology (DiNOGMl; DipTeC), IRCCS AOU S Martino-IST, Genoa, Italy; ${ }^{9}$ Dipartimento Emergenza e Trapianti d'Organi (D.E.T.O), University of Bari, Bari, Italy; ${ }^{10}$ Gerontology Research Group, Department of Medicine, Faculty of Health Sciences, University of A Coruña, A Coruña, Spain; ${ }^{11}$ Unit of Neurodegenerative Diseases, Department of Clinical Research in Neurology, University of Bari "Aldo Moro", Pia Fondazione Cardinale G. Panico, Tricase, Lecce, Italy; ${ }^{12}$ Department of Neurology and Neurophysiopathology, Hospital "Di Venere", Bari, Italy; ${ }^{13}$ Department of Imaging - Division of Radiology, Hospital "Di Venere", Bari, Italy; ${ }^{14}$ Division of Neuroradiology, "F. Ferrari" Hospital, Casarano, Lecce, Italy; ${ }^{15}$ Department of Radiology, Card. G. Panico Hospital, Tricase, Lecce, Italy; ${ }^{16}$ Department of Basic Medical Sciences, Neurosciences and Sense Organs, University of Bari "Aldo Moro", Bari, Italy; ${ }^{17}$ LENITEM (Laboratory of Epidemiology, Neuroimaging and Telemedicine), IRCCS Centro "S. Giovanni di Dio-F.B.F.", Brescia, Italy; ${ }^{18}$ Memory Clinic and LANVIE - Laboratory of Neuroimaging of Aging, University Hospitals and University of Geneva, Geneva, Switzerland Correspondence: Claudio Babiloni (claudio.babiloni@uniroma1.it) Neuropsychiatric Electrophysiology 2016, 2(Suppl 1):A19

\section{Background and aim}

In the European FP7 "DECIDE" project (www.eu-decide.eu), a computinggrid infrastructure was developed to compute electroencephalographic (EEG) and other biomarkers for diagnosis and instrumental assessment of patients with Alzheimer's disease (AD). In the framework of that project, previous evidence showed a $75.5 \%$ best accuracy in the classification of 120 Alzheimer's disease (AD) patients with dementia and 100 matched normal elderly (Nold) subjects based on cortical source current density and linear lagged connectivity estimated by eLORETA freeware from resting state eyes-closed electroencephalographic (rsEEG) rhythms (Babiloni et al., 2016). Specifically, that accuracy was reached using the ratio between occipital delta and alpha1 current density for a linear univariate classifier (receiver operating characteristic curves). Here we tested a nonlinear multivariate classification (artificial neural networks, ANNs) from the same database of rsEEG markers.

Methods

Frequency bands of interest of the mentioned EEG database were delta $(2-4 \mathrm{~Hz})$, theta $(4-8 \mathrm{~Hz})$, alpha1 $(8-10.5 \mathrm{~Hz})$, and alpha2 $(10.5-13 \mathrm{~Hz})$ as an input to ANNs.

Results

ANN classification showed an accuracy of $77 \%$ using the most 4 discriminative rsEEG markers of source current density (delta/alpha1 and theta/alpha1 ratios in posterior cortical lobes). It also showed an accuracy of $72 \%$ using the most 4 discriminative rsEEG markers of source lagged linear connectivity (alphas between posterior cortical lobes). With these 8 markers combined, an accuracy of $76 \%$ was reached. Overall, the present nonlinear (ANN) multivariate classification rate cross-validated that obtained using a linear univariate classifier.

\section{Conclusions}

Although these linear rsEEG markers of cortical activity and connectivity unveil different relevant neurophysiological mechanisms underpinning cortical arousal and vigilance in $A D$ patients, they provide quite redundant information for classification purposes. Future $A D$ studies should use ANNs combining the present markers with other linear (i.e. directed transfer function) and nonlinear rsEEG markers to improve the classification accuracy.

A20

Basmisanil, a negative allosteric modulator of GABA-A alpha5 subunit-containing receptors shows target and neuronal circuit engagement in man

Joerg F Hipp ${ }^{1}$, Robert Comley ${ }^{1}$, Darren Bentley ${ }^{4}$, Michael Derks ${ }^{4}$, Pilar Garces ${ }^{1}$, Frederic Knoflach ${ }^{2}$, Sian Lennon-Chrimes ${ }^{4}$, Stephane Nave ${ }^{1}$, Jana Noldeke', Nick Seneca', Gerhard Trube ${ }^{2}$, Christoph Wandel ${ }^{3}$, Andrew WThomas $^{5}$, and Maria-Clemancia Hernandez ${ }^{2}$

${ }^{1}$ Roche Pharma Research and Early Development, Translational Medicine Neuroscience and Biomarkers, F. Hoffmann-La Roche Ltd., CH-4070 Basel, Switzerland; ${ }^{2}$ Roche Pharma Research and Early Development, Discovery Neuroscience, F. Hoffmann-La Roche Ltd., CH-4070 Basel, Switzerland. ${ }^{3}$ Roche Pharma Research and Early Development, Clinical Safety, F. Hoffmann-La Roche Ltd., CH-4070 Basel, Switzerland; ${ }^{4}$ Roche Pharma Research and Early Development, Clinical Pharmacology, F. Hoffmann-La Roche Ltd., CH-4070 Basel, Switzerland; ${ }^{5}$ Roche Pharma Research and Early Development, Medicinal Chemistry, F. Hoffmann-La Roche Ltd., CH4070 Basel, Switzerland

Correspondence: Joerg F Hipp (joerg.hipp@roche.com)

Neuropsychiatric Electrophysiology 2016, 2(Suppl 1):A20

\section{Background}

Inhibitory GABAergic signaling plays a key role in brain function. Drugs that enhance GABA-A receptor function (e.g. Benzodiazepines) are widely used to treat conditions such as anxiety, insomnia, and epilepsy. In contrast, no inhibitors of GABA-A receptors exist for clinical use. Preclinical animal studies suggest that releasing inhibition by selectively inhibiting GABA-A alpha5 subunit-containing receptors may be beneficial in conditions of impaired cognition such Down syndrome, Schizophrenia, and Alzheimer's disease, and may also promote functional recovery after ischemic stroke, importantly without the side effects associated to non-selective inhibitors. Here we characterize basmisanil, a novel selective negative allosteric modulator of GABA-A alpha5 receptors, in terms of in vitro pharmacology as well as receptor occupancy and EEG signature in healthy volunteers.

Methods

Radioligand binding (3H-flumazenil) and voltage-clamp electrophysiology experiments were conducted in vitro on GABA-A receptors expressed in HEK293 cells and Xenopus oocytes to demonstrate binding and functional selectivity for the GABA-A alpha5 vs. alpha1/ $2 / 3$ subunit-containing receptors. A receptor occupancy study with the GABAA a5 PET tracer $\left[{ }^{11} \mathrm{C}\right] \mathrm{Ro} 15-4513$ was conducted in 10 healthy volunteers at 3 timepoints: baseline, 3 , and $9 \mathrm{~h}$ following 1 of 4 doses of basmisanil $(2 \times 15,2 \times 60,3 \times 130,3 \times 1250 \mathrm{mg})$. A separate EEG study in 12 volunteers measured at baseline, midazolam (5 mg), and 14 days of basmisanil treatment $(240 \mathrm{mg}$, bid).

Results

Basmisanil (RO5186582, RG1662) bound to cloned human GABA-A alpha5 with 5 -nM affinity and more than 90 -fold selectivity versus alpha $1 / 2 / 3$ subunit-containing receptors, and concentration-dependently and reversibly inhibited the GABA-induced current of alpha5 expressing cells, yet had weak or no activity on GABA-A receptors containing other alpha subunits. Using PET, receptor occupancy was confirmed in vivo in key regions of GABA-A alpha5 subunit containing receptors including hippocampus, insula, prefrontal cortex, and ventral striatum. Spectral analysis of the resting EEG revealed power increase in theta to alpha-, and decrease in the beta frequency range. This EEG signature was qualitatively opposite to that of Midazolam, a non-selective positive allosteric modulator of GABA-A receptors. Basmisanil was safe and well tolerated, and no treatment-emergent epileptiform abnormalities were observed. Discussion

Basmisanil is a highly selective GABA-A alpha5 negative allosteric modulator that reaches the desired target with good safety and tolerability, and modulates neuronal activity in humans. These data suggest basmisanil as a promising candidate drug for further clinical 
testing in conditions which may benefit from a reduction in excessive GABA-mediated tonic inhibition, such as cognitive impairment and stroke recovery.

Competing interests

All authors are or were employers of F. Hoffmann-La Roche Ltd.

\section{A21}

EEG cross-frequency coupling associated with attentional performance: an RDoC approach to attention

Berrie Gerrits ${ }^{1,2}$, Madelon A Vollebregt ${ }^{1,2}$, Sebastian Olbrich ${ }^{4}$, Roy PC Kessels ${ }^{2}$, Donna Palmer ${ }^{5}$, Evian Gordon ${ }^{5}$, Martijn Arns ${ }^{1,3}$

${ }^{1}$ Research Institute Brainclinics, Nijmegen, The Netherlands; ${ }^{2}$ Radboud University Medical Centre, Donders Institute for Brain, Cognition and Behaviour, Department of Cognitive Neuroscience, Nijmegen, The Netherlands; ${ }^{3}$ Dept. of Experimental Psychology, Utrecht University, Utrecht, The Netherlands; ${ }^{4}$ Dept. of Psychiatry, Psychotherapy and Psychosomatic, University Zürich, Zürich, Switzerland; ${ }^{5}$ Brain Resource Ltd., Sydney, NSW, Australia

Correspondence: Berrie Gerrits (b.gerrits@donders.ru.nl)

Neuropsychiatric Electrophysiology 2016, 2(Suppl 1):A21

The quality of attentional performance plays a crucial role in goaldirected behavior in daily life activities, cognitive task performance, and in multiple psychiatric illnesses. The Research Domain Criteria (RDoC) approach put forward by the National Institute of Mental Health aims to investigate cognitive constructs while abandoning the conventional diagnostic system of psychiatric illnesses. The current study used an RDoC approach to investigate functions underlying attentional performance.

One of the previously postulated physiologic mechanisms that could explain variance in attentional performance is the quality of interplay between neuronal networks ${ }^{1,2}$. Various attempts to visualize this interplay have been made using different approaches. In our current study, we aimed to validate the approach of functional Independent Component Analysis ( $\mathrm{flCA}$ ) based on electroencephalograms (EEG's) for this purpose. This method yields components that reflect EEG cross-frequency coupling patterns between networks (details about the method can be found elsewhere ${ }^{3}$ ).

We first applied fICA to combined Eyes Open resting state EEG and EEG during an $n$-back task data in a large sample of healthy adults $(n=1397)$, yielding 32 components. Secondly, we obtained individual component loadings for every subject for the two conditions as well as a difference loading score (Loading task -Loading ${ }_{\mathrm{EO}}$ ) per network. Thirdly, we operationalized attentional performance by differentiating between attenders $(n=$ 704) versus non-attenders, $(n=320)$ on the $n$-back task and found a significant difference between groups for the difference loading score for component 10 . We proposed that component 10 reflects the anticorrelated interaction of an attention network and a resting state network. This finding was cross-validated in an adolescent Attention-Deficit/Hyperactivity Disorder (ADHD) population $(n=80)$, clinically suffering from attentional problems. As expected, the difference loading scores in this group was similar to the pattern observed in non-attenders. Furthermore, it was accompanied by a lower overall loading on component 10 in both conditions.

The current findings seem to validate fICA as a method to visualize neuronal networks and their interactions. Combining this method with objective behavioral measures may contribute to the understanding of brain mechanisms involved in attention and attentional problems such as observed in multiple psychiatric illnesses.

\section{References}

1. Leech, R., Kamourieh, S., Beckmann, C. F., \& Sharp, D. J. (2011). Fractionating the default mode network: Distinct contributions of the ventral and dorsal posterior cingulate cortex to cognitive control. The Journal of Neuroscience: The Official Journal of the Society for Neuroscience, 31(9), 3217-24. doi: 10.1523/JNEUROSCI.5626-10.2011

2. Weissman, D. H., Roberts, K. C., Visscher, K. M., \& Woldorff, M. G. (2006). The neural bases of momentary lapses in attention. Nature Neuroscience, 9(7), 971-8. doi: 10.1038/nn1727
3. Aoki, Y., Ishii, R., Pascual-Marqui, R. D., Canuet, L., Ikeda, S., Hata, M., Takeda, M. (2015). Detection of eeg-resting state independent networks by eloreta-ica method. Frontiers in Human Neuroscience, 9. doi: 10.3389/fnhum.2015.00031

\section{A22}

\section{Attention for inhibition in ADHD: new insights with ERP source} imaging

Tieme WP Janssen ${ }^{1}$, Dirk J Heslenfeld ${ }^{1}$, Rosa van Mourik', Katleen Geladé $^{2}$, Athanasios Maras², Jaap Oosterlaan

${ }^{1}$ Clinical Neuropsychology, VU University, Amsterdam, Noord-Holland, 1081 BT, Netherlands; ${ }^{2}$ Yulius Academy, Barendrecht, Zuid-Holland, 2994 GC, Netherlands

Correspondence: Tieme WP Janssen (twp.janssen@vu.nl)

Neuropsychiatric Electrophysiology 2016, 2(Suppl 1):A22

\section{Background}

Deficits in response inhibition figure prominently in models of ADHD and have been documented in cognitive [1], ERP [2] and fMRI studies [3]. Parallel to these developments, some authors have criticized the inhibition model of ADHD and associated methodology, suggesting that attentional factors confound former results [4]. In a previous fMRI study [5] we aimed to control for attentional confounds during a stop-signal task (SST). Despite this modified SST, we found evidence for reduced activation in key-areas of the inhibition network, such as the right inferior frontal gyrus (rlFG), supplementary motor area (SMA) and anterior cingulate cortex (ACC). However, according to Barkley [6], inhibition problems precede other cognitive dysfunctions, such as attentional deficits. In order to investigate this hypothesis at the brain level, both high spatial and temporal resolution are needed, which have not yet been fully integrated in one imaging technique. In the current study [7], we addressed this issue by localizing ERP components associated with response inhibition in children with ADHD.

Methods

Dense array ERPs (128 electrodes) were obtained for 46 children with ADHD and 51 controls during the SST. Early and late components were compared between groups. N2 and P3 components were localized with LAURA distributed linear inverse solution for each participant, and statistically compared between groups (Bonferroni-corrected based on the number of electrodes, with $p=.05 / 128=.0004)$.

Results

A success-related N1 modulation was only apparent in the ADHD group. N2 and P3 amplitudes were reduced in ADHD. During the successful inhibition N2, the ADHD group showed reduced activation in rIFG, SMA, and right temporoparietal junction (rTPJ), and during failed inhibition in the rIFG. During the successful inhibition P3, reduced activation was found in ACC and SMA.

Conclusions

Source localization of N2 revealed not only a typical inhibition network (rIFG and SMA) that was affected, but also a major hub of the ventral attention system, the rTPJ. The ventral attention system supports attentional reorienting to salient and behaviourally relevant external stimuli. The fact that this ventral attention network is implicated in the same 50 ms time window (240-290 ms after stop stimulus) as the inhibition network creates a challenge to Barkley's theory of ADHD.

Competing interests

The author(s) declare no potential conflicts of interest

References

1. Oosterlaan, J., Logan, G. D. \& Sergeant, J. A. Response inhibition in AD/ $H D, C D$, comorbid AD/HD + CD, anxious, and control children: a metaanalysis of studies with the stop task. J. Child Psychol. Psychiatry. 1998; 39: 411-425.

2. Johnstone, S., Barry, R. \& Clarke, A. R. Behavioural and ERP indices of response inhibition during a Stop-signal task in children with two subtypes of Attention-Deficit Hyperactivity Disorder. Int. J. Psychophysiol. 2007; 66: 37-47.

3. Hart, H., Radua, J., Nakao, T., Mataix-Cols, D. \& Rubia, K. Meta-analysis of Functional Magnetic Resonance Imaging Studies of Inhibition and 
Attention in Attention-deficit/Hyperactivity Disorder. JAMA Psychiatry. 2013; 70: 185-198

4. Alderson, R., Rapport, M. D. \& Kofler, M. J. Attention-deficit/hyperactivity disorder and behavioral inhibition: a meta-analytic review of the stopsignal paradigm. J. Abnorm. Child Psychol. 2007; 35: 745-58.

5. Janssen, T. W. P., Heslenfeld, D. J., Mourik, R. van, Logan, G. D. \& Oosterlaan, J. Neural correlates of response inhibition in children with attention-deficit/hyperactivity disorder: A controlled version of the stopsignal task. Psychiatry Res. Neuroimaging. 2015; 233: 278-284.

6. Barkley, R. A. Behavioral inhibition, sustained attention, and executive functions: Constructing a unifying theory of ADHD. Psychol. Bull. 1997; 121: 65-94.

7. Janssen, T. W. P. et al. Alterations in the Ventral Attention Network During the Stop-Signal Task in Children With ADHD: An Event-Related Potential Source Imaging Study. J. Atten. Disord. 2015; Epub ahead of print

\section{A23}

Neurofeedback and pharmacological treatments in ADHD - evidence and EEG-markers

Daniel Brandeis ${ }^{1,2}$

${ }^{1}$ Department of Child and Adolescent Psychiatry and Psychotherapy, Central Institute of Mental Health, Medical Faculty Mannheim/ Heidelberg University, J5, 68159 Mannheim, Germany; ${ }^{2}$ Department of Child and Adolescent Psychiatry and Psychotherapy, Psychiatric Hospital, University of Zürich, Neumünsterallee 9, 8032 Zürich, Switzerland Neuropsychiatric Electrophysiology 2016, 2(Suppl 1):A23

\section{Scientific background/Introduction}

Neurofeedback is a promising treatment for ADHD despite recent evidence cautioning that probably blinded ratings draw a less positive picture.

\section{Theoretical framework/Hypothesis/Purpose of the work}

The current presentation will focus on how recent metaanalytic evidence for neurofeedback in ADHD, address specifically studies on the add-on use and interactions with pharmacological treatment, and on the predictive EEG-based markers for neurofeedback.

\section{Used Methods and Materials}

Review of evidence from recent metaanalysis during the last 8 years [1-3] and selected individual papers on the relation to pharmacological treatment, EEG markers $[4,5]$ and mechanism of learning self-regulation.

\section{Findings}

The clinically relevant efficacy of neurofeedback is reduced in probably blinded ratings and compared to active or sham control conditions. This may reflect considerable unspecific effects, compromised neurofeedback quality, or lack of learning self-regulation. How neurofeedback depends on previous or concurrent pharmacological treatment is unclear. Some head to head studies report comparable or additive effects of neurofeedback and medication, but not that neurofeedback works best as an add-on or second stage treatment (beyond practical considerations). Several predictive EEG markers have been proposed but await replication.

\section{Discussion of Relevance and Implications for future Research}

The findings do not yet allow for clear recommendations as to which patients profit most from neurofeedback alone or in combination pharmacological intervention. Studies on stepped care approaches and personalized approaches - what works for whom - are urgently needed.

\section{References}

1. Arns, M., et al., Efficacy of neurofeedback treatment in ADHD: the effects on inattention, impulsivity and hyperactivity: a meta-analysis. Clinical EEG and Neuroscience, 2009. 40(3): p. 180-189.

2. Cortese, S., et al., Neurofeedback for attention-deficit/hyperactivity disorder: meta-analysis of clinical and neuropsychological outcomes from randomized controlled trials. Journal of the American Academy of Child \& Adolescent Psychiatry, 2016. 55(6): p. 444-55.

3. Sonuga-Barke, EJ.S., et al., Nonpharmacological interventions for ADHD: Systematic review and meta-analyses of randomized controlled trials of dietary and psychological treatments. American Journal of Psychiatry, 2013. 170(3): p. 275-89.

4. Wangler, S., et al., Neurofeedback in children with ADHD: Specific eventrelated potential findings of a randomized controlled trial. Clinical Neurophysiology, 2011. 122(5): p. 942-50.
5. Arns, M., W. Drinkenburg, and J. Leon Kenemans, The Effects of QEEG Informed Neurofeedback in ADHD: An Open-Label Pilot Study. Applied Psychophysiology and Biofeedback, 2012. 7(3): p. 171-80.

A24

Deviant alpha oscillations as measure to help understanding the underlying mechanism of ADHD and predict treatment outcome Madelon A Vollebregt ${ }^{1,2}$, Martijn Arns ${ }^{1,3}$

${ }^{1}$ Research Institute Brainclinics, Nijmegen, The Netherlands; ${ }^{2}$ Department of Cognitive Neuroscience, Donders Institute for Brain, Cognition and Behaviour, Radboud University Medical Centre, Nijmegen, The Netherlands; ${ }^{4}$ Dept. of Experimental Psychology, Utrecht University, Utrecht, The Netherlands

Correspondence: Madelon A Vollebregt (madelon@brainclinics.com) Neuropsychiatric Electrophysiology 2016, 2(Suppl 1):A24

\section{Background}

Attention-deficit hyperactivity disorder (ADHD) is characterized by an inappropriate pattern of inattentiveness. Increasing evidence demonstrates that the modulation of alpha oscillations plays an important role in the allocation of attention. A failure to modulate alpha activity might therefore reflect ADHD. The first study presented here aimed to investigate alpha modulation in children with ADHD during attentional performance. The second study aimed to replicate and extend previous findings with respect to electroencephalographic (EEG) biomarkers that have shown promise in predicting treatment outcome to stimulant medication in ADHD.

Methods

For the first study [1], posterior alpha activity $(8-12 \mathrm{~Hz})$ was measured in 30 healthy children and 30 children with ADHD aged 7-10 years, using EEG while they performed a visuospatial covert attention task. We focused the analyses on healthy boys $(\mathrm{N}=9)$ and boys with ADHD $(\mathrm{N}=17)$. For the second study [2], data from the international Study to Predict Optimized Treatment Response in ADHD (iSPOT-A), 336 children and adolescents with ADHD were included and prescribed methylphenidate, and 158 healthy children were included. Treatment response was established after six weeks using the clinician rated ADHDRating Scale-IV (ADHD-RS-IV). Responders to treatment were defined as $>25 \%$ improvement. The EEG Theta/Beta ratio (TBR) and alpha peak frequency (APF) were investigated as predictors for treatment outcome. Results

In the first study, alpha activity in typically developing boys was similar to previous results of healthy adults: it decreased in the hemisphere contralateral to the attended hemifield, whereas it relatively increased in the other hemisphere. However, in boys with ADHD this hemispheric lateralization in the alpha band was not obvious (group contrast, $p=.018$ ). In the second study, male-adolescent non-responders exhibited a low frontal APF $(E S=0.83)$, whereas no differences in TBR were found between responders and non-responders. $62 \%$ of the ADHD group was classified as a responder. Responders were more often males (63\% versus $51 \%, p=.031)$, but did not differ from non-responders in age, medication dosage, and baseline severity of ADHD.

Conclusions

The first study demonstrated that the ability to modulate alpha oscillations in visual regions with the allocation of spatial attention was clearly present in healthy boys, but not in boys with ADHD. The second study demonstrated that male adolescent non-responders to methylphenidate display a lower frequency at which frontal alpha oscillations is peaking. The typical maturational changes in EEG emerging in adolescents observed in ADHD responders and controls, are absent in non-responders.

Trial registration

Clinical trial registration information; www.clinicaltrials.gov; NCT01932398 \& NCT00863499

\section{Competing interests}

MA reports research grants, options/shares from Brain Resource Ltd. (Sydney, Australia) and neuroCare group and he is also a co-inventor on 4 patent applications (A61B5/0402; US2007/0299323, A1; WO2010/139361 A1) related to EEG, neuromodulation and psychophysiology, but does not own these nor receives any proceeds related to these patents. 


\section{References}

1. Vollebregt, M.A., Zumer, J.M., ter Huurne, N., Buitelaar, J.K., Jensen, O. (2016). Posterior alpha oscillations reflect attentional problems in boys with Attention Deficit Hyperactivity Disorder. Clinical Neurophysiology, 127(5), 2182-2191.

2. Arns, M., Vollebregt, M.A., Palmer, D., Spooner, C., Gordon, E., Kohn, M., Clarke, S., Elliot, G., Buitelaar, J.K. (in prep). ADHD non-response to methylphenidate associated with slow alpha peak-frequency in male adolescents: Results from the iSPOT-A study.

\section{A25}

Brain arousal regulation: a predictive biomarker in psychiatry

Christian Sander, Ulrich Hegerl

Department of Psychiatrie, University Hospital Leipzig, Leipzig, Saxonia, 04103, Germany

Correspondence: Christian Sander (Christian.Sander@medizin.uni-leizig.de)

Neuropsychiatric Electrophysiology 2016, 2(Suppl 1):A25

Arousal fundamentally impacts normal and abnormal behavior, and recently research on disturbed arousal regulation in mental disorders has attracted increasing interest. Accordingly, arousal has been implemented as a basic dimension of mental disorders in the Research Domain Criteria (RDoC) project of the National Institute of Mental Health (NIMH). The talk introduces the arousal regulation model of affective disorders and ADHD, which suggests hyper-arousal as a core pathogenetic factor in uni- and bipolar depression, and, in contrast, hypo-arousal in mania and ADHD. The model explains different clinical phenomena, as manic behavior is in parts interpreted as an autoregulatory attempt to stabilize brain arousal by creating a stimulating environment, whereas the withdrawal and sensation avoidance as well as insomnia symptoms in depression is seen as reflecting the underlying chronic hyperarousal. Many depressed patients experience themselves as subjectively fatigued and in need of rest, extended bed-times and inactivity in most cases do not result in the desired recovery but in the contrary depressive symptoms and sleep problems tend to increase. As inferable from the model, interventions that decrease arousal (e.g. antidepressants) or increase sleep need (e.g. sleep deprivation, sleep restriction) are efficient, whereas the inefficacy of stimulants has often been shown in depression. The arousal model contributes to delineating more homogenous subgroups within affective disorders and predicts response to treatment based on the respective brain arousal disturbance. Electroencephalography under resting conditions is most suitable for the assessment of brain arousal regulation, as different arousal states (also called EEG-vigilance stages) can be differentiated during the transition from high alertness to drowsiness until sleep onset according to specific EEG characteristics. The second part of the talk will introduce a computeralgorithm (Vigilance Algorithm Leipzig, VIGALL2.1), allowing semiautomatic classification of EEG-vigilance stages during restingEEG recordings. The time sequence of these EEG-vigilance stages indicates the individual arousal regulation of the recorded subject. The final part of the talk will outline results from current studies applying VIGALL 2.1 and investigating hypotheses derived from the arousal regulation model with regards to the usage of brain arousal regulation as a diagnostic and/or predictive biomarker in psychiatric research.

\section{Competing interests}

The author(s) declare(s) that they have no competing interests.

\section{A26}

Panic attacks on the epilepsy spectrum

Nash N Boutros (boutrosn@umkc.edu)

University of Missouri-Kansas City, Kansas City, Missouri, USA 64108

Neuropsychiatric Electrophysiology 2016, 2(Suppl 1):A26

\section{Background}

A rather highly unexamined dimension of psychiatric symptomatology is duration particularly when ultra brief and episodic. Brief and episodic symptoms are frequently seen in individuals committing seemly unmotivated repeated violent acts, dissociative episodes or panic attacks. Growing evidence suggest that at least in a subset of patients exhibiting these symptoms focal fronto-limbic hyper-excitability may be contributing to the clinical picture. Such conditions can be conceptualized to fall on the epilepsy spectrum. We now present preliminary data from panic patients examining their focal coherence.

Methods

11 panic patients and 10 healthy control subjects were included. All had at least two unprovoked panic attacks. All subjects underwent $10 \mathrm{~min}$ of eye open and 10 min of eyes closed magnetoencephalography recording (CTF, Canada). The data were analyzed based on 27 regions of interests in each hemisphere. Areas were divided to fronto-limbic (FL) or extra fronto-limbic (EFL).

Findings

In the eyes-closed condition one pair had higher coherence in the control group (Occipital-Parietal). 31 pairs had higher coherence in PD (20 FL-FL, 11 FL-EFL, NONE EFL-EFL). In the eye open condition NO area showed more coherence in controls while 59 did for panic. Of the 59 pairs 46 were FL-FL, 15 were FL-EFL and NONE were EFL-EFL.

Relevance and Implications for Future Research

If the above data are confirmed in future studies with larger sample sizes, the implications would be that it may be possible to predict response to various types of medications (e.g., selective serotonin reuptake inhibitors (SSRIs) vs. anticonvulsants). These findings would lead to more personalized treatment planning.

\section{A27}

Electrophysiological markers in the prediction of various treatment approaches in major depression and obsessive compulsive disorder

Sebastian Olbrich', Alexandra Kirsten', Anna-Lena Dohrmann², Galina Surova² ${ }^{1}$ Department for Psychiatry, Psychotherapy and Psychosomatic,

University of Zurich, Zurich, Switzerland; '2Department for Mental Health, University Leipzig, Leipzig, Germany

Correspondence: Sebastian Olbrich (Sebastian.olbrich@puk.zh.ch)

Neuropsychiatric Electrophysiology 2016, 2(Suppl 1):A27

Despite large research efforts - also in electrophysiology - within the last decades, up to now there are no clinically accepted or used biomarkers that help to diagnose affective or anxiety disorders: It seems that the discriminative abilities of clinicians concerning diagnostic decisions are fair enough. However, what is even more important than a correct diagnosis is choosing the best treatment. Here, the decisions of the clinicians are not supported by any evidence. Especially in disorders where with various treatment approaches - such as major depressive disorder (MDD) and obsessive compulsive disorder (OCD) - but a still very high percentage of non-responders to first line therapies, biomarkers could contribute to an individualized medicine with faster responses and less trial and error approaches.

Electrophysiological biomarkers provide a direct window to brain function combined with cost-effective settings and broad availability. Therefore, data on electroencephalogram (EEG) based algorithms derived from different studies will be presented that help to discriminate patients with better response to different types of treatment. Within the first part, the focus will be on predictors for treatment outcome following therapy with different types of antidepressants. Further, EEG-based biomarkers with discriminative power concerning outcome of electro-convulsive therapy (ECT) and will be shown. The second part is dedicated to treatment of OCD with cognitivebehavioral therapy, selective-serotonin inhibitors (SSRIs) or a combination of both. EEG-based biomarkers will be presented that might support the right choice of treatment.

The presentation of data will be followed by a brief outlook to what will has to come to transfer research knowledge into every day routine. 
Besides the need for large, prospective multicenter studies, new analytical approaches will be presented that could help to establish an individualized medicine in neuropsychiatric disorders one day.

\section{Competing interests \\ Authors report no competing interests.}

A28

The sgACC in depression: getting at the heart of it

TA Iseger ${ }^{1,2}$, JL Kenemans ${ }^{1}$, M Arns ${ }^{1,2,3}$

${ }^{1}$ Dept. of Experimental Psychology, Utrecht University, Utrecht, The Netherlands; ${ }^{2}$ Research Institute Brainclinics, Nijmegen, The Netherlands; ${ }^{3}$ neuroCare Group, Munich, Germany

Correspondence: TA Iseger (tabitha@brainclinics.com)

Neuropsychiatric Electrophysiology 2016, 2(Suppl 1):A28

Major depressive disorder (MDD) is a chronic, mental disease with a remitting and relapsing course. Antidepressant medication is the most common treatment for MDD, however, the precise working mechanism underlying these treatments remains unclear. Recent neuromodulation treatments demonstrate that direct stimulation of the dorsolateral prefrontal cortex (DLPFC) and subgenual anterior cingulate (sgACC) relate to clinical improvement, suggesting connectivity alterations in this network to mediate antidepressant response, which might be similar for pharmacological treatments as well. This will be the focus of part 1. The international Study to Predict Optimized Treatment in Depression (iSPOT-D) is an international multicenter study that collected EEG data for 1008 MDD patients. We investigated whether connectivity in alpha and theta frequencies within this network changed during antidepressant treatment between: patients and controls, and responders and non-responders. Women exhibited higher alpha and theta connectivity compared to males, both pre- and posttreatment. Decreased alpha connectivity after treatment was found only for male responders, while non-responders and females exhibited no changes in alpha connectivity. Furthermore, it could be useful to $a$ priori stratify by gender for future MDD studies [1].

Part two focusses on functional connectivity assumptions between the DLPFC, sgACC and the vagal nerve. Preliminary results will be presented regarding a method to localize the DLPFC: neuro-cardiacguided rTMS (NCG-rTMS). The efficacy of rTMS in the treatment of MDD has been well established in recent years, however with various methods of locating the DLPFC. It has been proposed that the efficacy of rTMS in MDD is more related to stimulating the area that is functionally connected to the sgACC rather then to specific cortical areas. Therefore, we set-out to develop and test a new method that employs the functional role of the sgACC to establish in real time if the correct cortical area is targeted. Several studies have shown that the sgACC is involved in parasympathetic regulation such as heart rate (HR) and respiration, and that neurostimulation of these areas led to HR decreases, most likely through connectivity with the nervus vagus. Based on the notion that rTMS aims to transsynaptically stimulate the sgACC, we used electrocardiogram (ECG) R-peak triggered single pulse TMS to various frontal locations to establish the correct DLPFC location.

\section{References}

[1] T.A. Iseger, M.S. Korgaonkar, J.L. Kenemans, S.M. Grieve, C. Baeken, P. Fitzgerald \& M. Arns. Alpha connectivity change between the Subgenual Anterior Cingulate and Prefrontal Cortices related to antidepressant medication response in males. Submitted, Aug 2016

\section{A29}

Heart rate variability and sleep EEG derived markers as correlates of depression and treatment response

Marcel A Pawlowski ${ }^{1}$, Marek Adamczyk ${ }^{2}$, Thorsten Mikoteit ${ }^{2}$, Axel Steiger ${ }^{1}$ ${ }^{1}$ Max Planck Institute of Psychiatry, Munich, Germany; ${ }^{2}$ Psychiatric Clinics of the University of Basel, Center for Affective, Stress and Sleep Disorders, Basel, Switzerland

Correspondence: Marcel A Pawlowski (pawlowski@psych.mpg.de) Neuropsychiatric Electrophysiology 2016, 2(Suppl 1):A29

\section{Study objectives}

The relevance of rapid eye movement (REM) sleep for affective disorders derives from its well-established abnormalities in depressed patients, that is increased REM sleep pressure with increased frequency of rapid eye movements (REM density) as a trait marker of major depression. In this study we examined whether prefrontal theta cordance and heart rate variability (HRV) during REM sleep could represent biomarkers of antidepressant treatment response to optimize treatment outcome.

Methods

In an open-label, case-control design, thirty-three in-patients (21 females) with a depressive episode were treated with various antidepressants for four weeks. Response to treatment was defined as $a \geq$ $50 \%$ reduction of HAM-D score at the end of the fourth week. SleepEEG was recorded after the first and the fourth week of medication. Cordance was computed for prefrontal EEG channels in the theta frequency band during tonic REM sleep. HRV was derived from 3-min artefact-free electrocardiogram segments during REM sleep.

Results

First, fourteen responders had significantly higher prefrontal theta cordance as compared to nineteen non-responders after the first week of antidepressant medication. Second, HRV in REM sleep was decreased in depressive patients at week four as compared to controls (high effect size; Cohen's $d>1$ ). Third, HRV showed negative correlation with REM density in healthy subjects and patients at week four.

Conclusions

Our data suggest that prefrontal REM sleep-deprived cordance may predict response to antidepressant treatment in depressed patients, whereas HRV distinguishes healthy subjects from depressed patients.

\section{A30}

\section{Applying integrated EEG-behavioural analyses in genetic mouse} models for Autism Spectrum Disorder; the identification of translational neuronal biomarkers

Martien J Kas ${ }^{1,2}$, Karlijn I van Aerde ${ }^{2}$

${ }^{1}$ Groningen Institute for Evolutionary Life Sciences, University of

Groningen, Groningen, the Netherlands; ${ }^{2}$ Department of Translational

Neuroscience, University Medical Center Utrecht, Utrecht, the Netherlands

Correspondence: Martien J Kas (m.j.h.kas@rug.nl)

Neuropsychiatric Electrophysiology 2016, 2(Suppl 1):A30

Autism Spectrum Disorder (ASD) is a highly heterogeneous neurodevelopment disorder that is clinically defined by impaired social interaction, as well as repetitive and restricted behaviours. More than 200 ASD risk genes have been identified. Understanding the functional impact of these genetic variants will contribute to unravel clinical heterogeneity and to improve treatment efficacy. To provide clinically relevant neuronal biomarkers for specific biological subgroups, we initiated integrated EEG-behavioural analyses in selected genetic mouse models for ASD. So far, EEG data from ASD patients have shown changes in the power of brain oscillations in several frequency bands as well as in response to sensory stimuli. Although these results tell us that ASD patients have alterations at the level of brain circuits, and/or during processing of specific tasks, these biomarkers are currently not used for diagnosis of the disease.

To increase the selectively and sensitivity of neurophysiological biomarkers we analyzed the EEG of genetically modified mice during specific aspects of a behavioral task. To this end we coupled the systems used for EEG recordings and the software that tracks the location of the animal, allowing us to select parts of EEG measurements based on animal behaviour. We used Protocadherin 9 (Pcdh9) mutant mice as a model for the social interaction deficits seen in ASD patients (Bruining et al., 2015). These mice show deficits in sensorimotor development and long-term social discrimination capacity, while long-term fear conditioning is normal. Preliminary EEG results showed a decrease in gamma band oscillations in mutant mice during social interaction with both familiar and novel intruder mice. Furthermore, mutant mice showed impaired sensory information processing during an auditory mismatch negativity task. Currently, we are expanding these findings and investigate both EEG 
measurements and behavioral outcomes in experimental paradigms with varying degrees of difficulty for social discrimination. This way, we hope to identify translational EEG biomarkers to guide ASD patient classification and to contribute to the development of novel treatment strategies for this heterogeneous neurodevelopmental disorder.

\section{A31}

\section{Anesthesia, an opportunity to measure a pharmaco-EEG par} excellence

Clementina M van Rijn' ${ }^{1}$ Mariijte LA Jongsma², Philip LC van den Broek', Jan van Egmond ${ }^{3}$

'Donders Institute for Brain, Cognition and Behaviour, Radboud University, Nijmegen, the Netherlands; ${ }^{2}$ Behavioural Science Institute, Radboud University, Nijmegen, the Netherlands; ${ }^{3}$ Department of Anesthesiology, Radboud University Hospital, Nijmegen, the Netherlands Correspondence: Clementina M van Rijn (t.vanrijn@donders.ru.nl) Neuropsychiatric Electrophysiology 2016, 2(Suppl 1):A31

The IPEG is an association for researchers involved in electrophysiological brain research and pharmacology, and the contribution of pharmaco-EEG research to the field of neuroscience is gaining in importance. In the past few years the functions of brain circuits, i.e. functional neuroanatomical resting-state networks, have come to be on the verge of being understood. This progress is the result of close collaboration between many disciplines: neuroanatomy, psychology, physics and pharmacology, to name just a few, which are making a joint effort to understand the functioning of the brain.

In view of these developments, the EEG measured during anesthesia might hold keys to disentangle (or to the contrary perhaps to unify), behavioral, pharmacological and neurophysiological signatures of various states of behavior, especially of the difficult to quantify states of consciousness. This is because anesthesia is a drug-induced state in which patients do not have any sensation, they are unconscious. Moreover, during the whole period of anesthesia, the anesthesiologist meticulously monitors the state of wakefulness, so this procedure complies perfectly with the IPEG recommendation, which advices to measure EEG activity under vigilance-controlled conditions [4].

To induce a state of anesthesia, a variety of drugs can be used, all with quite different molecular targets. One of the still unanswered questions is: are different drugs inducing different states of anesthesia, or is anesthesia a well-described state that might be induced by modifying different stations in a hypothesized "esthesia circuit"? The contribution of mathematicians to the field of time series analysis is yielding advanced analysis algorithms with a huge potential to answer this question, since it touches on brain circuits and connectivity.

In this oral I will mini-review the literature to point out characteristic EEG and connectivity changes induced by various types of anesthetics, propofol, isoflurane and ketamine included [e.g.1,5]. I will illustrate the findings in the literature with our own data of both rats and humans $[2,3]$. Further research questions will be proposed and discussed with the audience, in the hope to boost interest and research in our IPEG society in the EEG under anesthesia, the pharmaco-EEG par excellence.

\section{Competing interests}

None.

\section{References}

1. Akeju O, Song AH, Hamilos AE, Pavone KJ, Flores FJ, Brown EN, Purdon $P L$. Electroencephalogram signatures of ketamine anesthesia-induced unconsciousness. Clin Neurophysiol, 2016, 127, 2414-2422.

2. Broek PLC van den, van Rijn CM, Egmond I van, Coenen AML, Booij LHDJ. An Effective Correlation Dimension and Burst Suppression Ratio of the EEG in rat. Correlation with Sevoflurane Induced Anaesthetic Depth. Eur J Anaesthesiology, 2006, 23, 391-402.

3. Broek, PLC van den, Monitoring Anesthetic Depth: Modification, Evaluation and Application of the Correlation Dimension. Nijmegen 2003 (thesis : http://repository.ubn.ru.nl/handle/2066/19249).
4. Drinkenburg WHIM, $\cdot$ Ruigt GSF; Ahnaou A. Pharmaco-EEG Studies in Animals: An Overview of Contemporary Translational Applications. Neuropsychobiology, 2015, 72, 151-164.

5. Purdon PL, Sampson A, Pavone KJ, Brown EN. Clinical Electroencephalography for Anesthesiologists: Part I: Background and Basic Signatures. Anesthesiology, 2015, 123(4):937-960

A32

Proof of early disintegration of functional network connectivity in the K18 seeding transgenic mouse model of tauopathy spreading from the locus coeruleus: novel opportunities for assessing pharmacological intervention therapies for Alzheimer's disease

A Ahnaou, C Walsh, L Raeymaekers, R Biermans, NV Manyakov, C

Wintmolders, A Bottelbergs, K Van Kolen, D Moechars, JA Kemp and WH

Drinkenburg

Department of Neuroscience Discovery, Janssen Research \&

Development, a Division of Janssen Pharmaceutica NV. Turnhoutseweg

30, B-2340 Beerse, Belgium

Correspondence: A Ahnaou (aahnaou@its.jnj.com)

Neuropsychiatric Electrophysiology 2016, 2(Suppl 1):A32

Alzheimer's disease (AD) is a disconnection syndrome manifested by the disruption of white matter integrity, loss of synapses and functional connectivity (FC) across different cortical and subcortical regions. Early in $A D$ progression, tau pathology can be found in the brainstem locus coeruleus (LC) prior to its amyloid-induced exacerbation and clinical symptoms. Accordingly, the pathological process of $A D$ is characterized by the cell-cell spread of tau pathology from the LC into the medial temporal lobe, which triggers pathological changes causing functional disconnectivity. Network dynamics have become a leading model to assess both the anatomical relationships (structural networks) and the coupling of dynamic neurophysiology (functional networks) linking separate brain regions.

The present study used a tau seeding model in which preformed synthetic tau fibrils (K18) were unilaterally injected into the LC of transgenic mice expressing mutant human P301L tau, equipped with multichannel electrodes in frontal cortical and CA1-CA3 hippocampal areas. This approach allows us to 1 /quantify longitudinal coherence and FC using phase-amplitude theta-gamma coupling (PAC); 2/identify the directionality of connectivity, using lagged and extended partial direct coherence (PDC); 3/measure pre-attentive auditory P50 potentials; 4/investigate sleep-wake organization; and 5/quantify phospho(p)-tau pathology in regions of interest using immunohistochemistry (AT100 antibody).

At the functional level, a decrease in spectral power at a range of frequencies in the hippocampal regions ipsilateral to the injection site is found at 2 weeks post-K18 injection, while an increase in power in contralateral hippocampal regions is hypothesized to be indicative of early compensatory mechanisms. Inter-hippocampal coherence is reduced in slow frequency oscillations and FC is significantly impaired as evidenced by: decreased intra- and interhemispheric hippocampal directionality of theta frequency oscillations; and reduced intra- and inter-hemispheric functional PAC strength. At the structural level, abnormal pTau aggregation is regionally specific, with AT100-positive tau detected in the pons, medulla, thalamus and cerebellum.

Ongoing assessment of pre-attentive auditory information processing, sleep-wake alterations and changes in the activity of GABAergic interneurons, which play a critical role in theta-gamma interactions, will allow further investigation into this aforementioned network dysfunction. Electrophysiological abnormalities in the hippocampus and cortex following injection of K18 into the LC convincingly support the relevance of tau pathology early in the LC. These functional alterations offer a reliable in vivo assay to test $A D$ therapeutic agents for early intervention of tau pathology and possible prevention of the impairments in synaptic plasticity and neuronal network connectivity as seen in $A D$. 
A33

Public-private initiative to align EEG biomarkers of Alzheimer's disease in human and mouse models for early stages of drug discovery: the achievements of IMI PharmaCog project

Claudio Babiloni ${ }^{1,2}$, Claudio Del Percio ${ }^{2,3}$, Nicola Marzano ${ }^{2}$, Susanna Cordone ${ }^{2}$ Susanna Lopezz" Giuseppe Noce ${ }^{6}$, Cristina Bagnolit, Paolo Maria Rossini ${ }^{5}$, Andrea Soricellii, Flavio Mariano Nobili', David Bartres Faz ${ }^{8}$, Olivier Blin', Pierre Payoux ${ }^{10}$, Regis Bordet ${ }^{11}$, Bernhard Mueller ${ }^{12}$, Magda Tsolaki ${ }^{13}$, Lucilla Parnetti ${ }^{14}$, Ulrich Heger ${ }^{15}$, Tilman Hensch ${ }^{15}$, Juergen Dukart ${ }^{16}$ Alessandro Bertolino $^{16}$, Gianluigi Forloni ${ }^{17}$, Angelisa Frasca ${ }^{17}$, Jill Richardson ${ }^{18}$, Jesper Frank Bastlund $^{19}$, Bettina Clausen ${ }^{19}$, Marina Bentivoglio ${ }^{20}$, Paolo Francesco Fabene ${ }^{20}$, Giuseppe Bertini ${ }^{20}$, Jonathan Kelley ${ }^{21}$, Wilhelmus Drinkenburg ${ }^{21}$, Giovanni Frisoni'; on behalf of PharmaCog Consortium

${ }^{1}$ Sapienza University of Rome, Rome, Italy; ${ }^{2}$ University of Foggia, Foggia, Italy; ${ }^{3}$ RCSS San Raffaele Pisana, Rome, Italy; ${ }^{4}$ RCCS Istituto Centro San Giovanni di Dio-Fatebenefratelli, Brescia, Italy; ${ }^{5}$ Catholic University, Rome, Italy; ${ }^{6}$ RCCS SDN, Naples, Italy; ${ }^{7}$ University of Genoa, Genoa, Italy; ${ }^{8}$ University of Barcelona, Barcelona, Spain; ${ }^{9}$ Mediterranean Institute of Cognitive Neurosciences, Marseille, France; ${ }^{10}$ University of Toulouse, Toulouse, France; ${ }^{11}$ Lille 2 University, Lille, France; ${ }^{12}$ University of Duisburg-Essen, Essen, Germany; ${ }^{13}$ University of Thessaloniki, Thessaloniki, Greece; ${ }^{14}$ University of Perugia, Perugia, Italy;

${ }^{15}$ University of Leipzig, Leipzig, Germany; ${ }^{16}$ Roche, Basel, Switzerland; ${ }^{17}$ Mario Negri Institute, Milan, Italy; ${ }^{18}$ GSK R\&D, London, UK; ${ }^{19}$ Lundbeck, Valby, Denmark; ${ }^{20}$ University of Verona, Verona, Italy; ${ }^{21}$ Janssen Pharmaceutica, Beerse, Belgium

Neuropsychiatric Electrophysiology 2016, 2(Suppl 1):A33

\section{Background and aim}

In the European FP7 IMI "PharmaCog" project (Grant Agreement $\mathrm{n}^{\circ}$ 115009, www.pharmacog.org), we evaluated whether cortical electroencephalographic (EEG) rhythms in quiet wakefulness reflected prodromal Alzheimer's disease (AD) in amnesic mild cognitive impairment (aMCI) and had a back-translational value in transgenic mouse models of Alzheimer's disease (AD).

Methods

The research data (including human biological samples) were sourced ethically and used in line with international ethical standards. EEG rhythms were recorded in $127 \mathrm{aMCl}$ subjects. Cortical sources of EEG rhythms were estimated by eLORETA package (http:// www.uzh.ch/keyinst/loreta.htm). Back translation of the EEG markers was tested on on- going EEG rhythms in wild type and transgenic mouse models of $A D$ developing an accumulation of $A B 1-42$ in the brain (i.e. one mutation in PDAPP and two mutations in TASTPM).

Results

(1) Compared with the aMCl sub-group showing "negativity" to $A \beta 1$ 42/phospho tau in the cerebrospinal fluid, the aMCl sub-group showing "positivity" (prodromal AD) exhibited an abnormal delta $(<4 \mathrm{~Hz})$ source activity in widespread cortical regions while a posterior source activity in low-frequency alpha rhythms $(8-10.5 \mathrm{~Hz})$ pointed to a progressive abnormality across disease progression in 2 years; (2) Ongoing EEG rhythms in the same frequency range were abnormal in the transgenic PDAPP and TASTPM mice when compared to the control wildtype animals. Furthermore, these EEG rhythms were modulated by an $A \beta 1-42$ lowering agent (monoclonal antibody 3D6) administered for 4 weeks in TASTPM mice. No effect was observed in wild-type mice.

\section{Conclusions}

The results of the PharmaCog project suggest that markers of on-going cortical EEG rhythms $<12 \mathrm{~Hz}$ may reflect prodromal $A D$ processes in $\mathrm{aMCl}$ subjects and can be back-translated to transgenic mouse models of $A D$. These results encouraged the use of EEG biomarkers for an early evaluation of new $A D$ modifying drugs in transgenic mouse models of $A D$.

\section{A34}

Neurophysiological biomarkers in first episode psychosis

Dean F Salisbury, Brian A Coffman, Timothy Murphy, Sarah M Haigh Department of Psychiatry,Dean F Salisbury University of Pittsburgh School of Medicine, Pittsburgh, PA, 15213, USA

Correspondence: Dean F Salisbury (salisburyd@upmc.edu)

Neuropsychiatric Electrophysiology 2016, 2(Suppl 1):A34
A biomarker is an objective biological measure that can be used for health risk prediction, or for screening, diagnosis, or tracking disease progression. In psychosis research, a key recent development has been the application of a staging framework. The understanding that there is a progressive course to psychosis, including a potential prodromal phase of increasing disability before the emergence of frank psychosis, has spurred the search for screening biomarkers to indicate those truly prodromal clinical high risk for psychosis individuals that will convert to psychosis (approximately $30 \%$ in 3 years), crucial for early prophylactic treatment. Practically, true prodromal cases are rare and studies tracking conversion long and costly. To be useful for screening any disease presence biomarker must obligatorily be reduced at first psychosis. Our group has been testing various auditory-based neurophysiological tasks in first episode psychosis individuals to develop screening biomarkers for an incipient psychotic break. Here we describe several candidate biomarkers. Passive listening, simple mismatch negativity (MMN) to a rare deviant tone is not abnormal in first episode psychosis (Study 1: 29 first episode schizophrenia and 40 controls; Study 2: 35 first episode schizophrenia and 35 controls). Complex MMN tasks that depend on extraction of patterns in auditory sequences show more promise. In a series of passive tasks where the number of tones in groups were occasionally changed, we saw significant reductions of complex MMN to a rare extra tone (19 first episode schizophrenia and 19 controls). We also observed abnormalities of a slow potential that appeared to indicate the formation of each group as an acoustic object. Having participants actively count stimuli in each group revealed that a missing tone (e.g. a group of 3 instead of a group of 4) elicited an emitted P300, and that the posterior P300b component was abnormal in first episode schizophrenia (20 first episode schizophrenia and 32 controls). Using a single tone auditory evoked potential task, attention to stimuli was manipulated by having participants either press a button to every 7 tones or watch a silent video (10 first episode schizophrenia and 10 matched controls). Healthy participants modulated their N100 with attention, but first episode psychosis individuals did not. These data suggest that several neurophysiological measures may be suitable as biomarkers for the presence of psychosis. Future work will deploy these tasks in clinical high risk individuals to track whether they show promise as screening biomarkers for an incipient psychotic break.

A35

Perception of sleep in patients with insomnia related to generalized anxiety disorder, patients with apnea and in healthy controls

Peter Anderer ${ }^{1,2}$, Georg Gruber ${ }^{1}$, Silvia Parapatics', Gerda M Saletu-Zyhlarz ${ }^{2}$, Bernd Saletu ${ }^{2}$, Georg Dorffner ${ }^{1,3}$

${ }^{1}$ The Siesta Group Schlafanalyse GmbH, Vienna, Austria; ${ }^{2}$ Department of Psychiatry and Psychotherapy, Medical University of Vienna, Vienna,

Austria; ${ }^{3}$ Center for Medical Statistics, Informatics and Intelligent Systems, Medical University of Vienna, Vienna, Austria

Correspondence: Peter Anderer (peter.anderer@thesiestagroup.com) Neuropsychiatric Electrophysiology 2016, 2(Suppl 1):A35

\section{Background}

Although sleep research has been trying to elucidate the relations between objective and subjective sleep and awakening quality for five decades, findings have often been controversial. This might be due to the lack of large data sets, to the differences between different sleep disorders and to the inter-individual differences in sleep perception per se.

Objectives

The aim of the present study was to investigate relations between objective and subjective sleep variables in a large number of healthy subjects as well as in 2 clinically relevant patient groups, i.e. nonorganic insomnia in generalized anxiety disorder (insomnia/GAD) and sleep apnea. 


\section{Material and methods}

One hundred and seventy-seven healthy subjects (94 females, 83 males, aged $20-95$ years), 61 insomniac GAD patients ( 32 females, 29 males, aged 21 - 66 years) and 51 apnea patients (7 females, 44 males, aged $29-73$ years) underwent two polysomnographic nights analyzed by the Somnolyzer [1] and completed the self-rating scale for sleep and awakening quality [2].

Results

Patients with insomnia/GAD underestimated their sleep efficiency in both nights (objective sleep efficiency index (SEI) $77 \%$ and $84 \%$ versus subjective SEI $57 \%$ and $64 \%$ for adaptation and baseline night, respectively, $\mathrm{p}<0.001$ Wilcoxon test). Apnea patients showed no differences between subjective and objective SEl (objective $80 \%$ and $87 \%$ versus subjective $79 \%$ and $86 \%$ ). Healthy controls - specifically males and subjects older than 60 years - overestimated their sleep efficiency in the adaptation night (objective $80 \%$ and $86 \%$ versus subjective $84 \%$ and $87 \%, p<0.001$ for night 1 ). Correlation analysis between objective and subjective SEl on change values from adaptation to baseline night revealed highly significant correlations for all three groups ( $r=.77$ for GAD, $r=.57$ for apnea and $r=51$ in healthy controls). Interestingly, the regression lines go through the origin in all three groups, i.e. no change in objective SEl is perceived as no change in subjective SEI.

\section{Conclusions}

Relations between subjective and objective sleep efficiency are influenced by age, gender and the type of sleep disorder. In correlation analyses, the problem of inter-individual judgements of sleep perception can be reduced by using change values between adaptation and baseline nights rather than raw values. The variety of correlations requires a parallel evaluation of subjective and objective variables as they are not interchangeable.

\section{Competing interests}

Peter Anderer, Georg Gruber, Silvia Parapatics and Georg Dorffner are employees of The Siesta Group Schlafanalyse GmbH. Gerda M Saletu-Zyhlarz and Bernd Saletu have no competing interests.

\section{References}

1. Anderer $\mathrm{P}$, et al. An E-health solution for automatic sleep classification according to Rechtschaffen and Kales: validation study of the Somnolyzer $24 x$ 7 utilizing the Siesta database. Neuropsychobiology. 2005; 51:115-133.

2. Saletu B, et al. (1997): Nonorganic insomnia in generalized anxiety disorder. 2. Comparative studies on sleep, awakening, daytime vigilance and anxiety under lorazepam plus diphenhydramine (SomniumR) versus lorazepam alone, utilizing clinical, polysomnographic and EEG mapping methods. Neuropsychobiology, 1997; 36:130-152.

\section{A36}

\section{Catecholaminergic regulation of learning rate in a dynamic} environment

Marieke Jepma, Sander Nieuwenhuis

Cognitive Psychology Unit, Leiden University, Leiden, the Netherlands

Correspondence: Marieke Jepma (m.jepma@fsw.leidenuniv.nl)

Neuropsychiatric Electrophysiology 2016, 2(Suppl 1):A36

Adaptive behavior in a changing world requires flexibly adapting one's rate of learning to the rate of environmental change. Recent studies have examined the computational mechanisms by which various environmental factors determine the impact of new outcomes on existing beliefs (i.e., the 'learning rate'). However, the brain mechanisms, and in particular the neuromodulators, involved in this process are still largely unknown. The brain-wide neurophysiological effects of the catecholamines norepinephrine and dopamine on stimulus-evoked cortical responses suggest that the catecholamine systems are well positioned to regulate learning about environmental change, but more direct evidence for a role of this system is scant. Here, we report evidence from a study employing pharmacology, scalp electrophysiology and computational modeling $(\mathrm{N}=32)$ that suggests an important role for catecholamines in learning-rate regulation. We found that the P3 component of the EEG-an electrophysiological index of outcome-evoked phasic catecholamine release in the cortex-predicted learning rate, and formally mediated the effect of prediction-error magnitude on learning rate. P3 amplitude also mediated the effects of two computational variablescapturing the unexpectedness of an outcome and the uncertainty of a preexisting belief-on learning rate. Furthermore, a pharmacological manipulation of catecholamine activity affected learning rate following unanticipated task changes, in a way that depended on participants' baseline learning rate. Our findings provide converging evidence for a causal role of the human catecholamine systems in learning-rate regulation as a function of environmental change.

\section{A37}

\section{Dopamine and the cortical representation of reward}

Iris Schutte, Ivo Heitland, J Leon Kenemans

Department of Experimental Psychology, Helmholtz Institute, Utrecht

University, Utrecht The Netherlands

Correspondence: Iris Schutte (i.schutte@uu.nl)

Neuropsychiatric Electrophysiology 2016, 2(Suppl 1):A37

The way humans behave is greatly affected by the principle expected utility, the combination of subjective value (SV) of the outcome of an act (is it rewarding?) and subjective probability (SP) of that outcome. In an initial study $(n=42)$ we examined the electro-cortical representations of the anticipation of SV and SP during a cued Go/NoGo experiment. During this task cue letters signaled upcoming target letters to which participants had to respond. The probability of target letter appearance after the cue letter and the amount of money that could be won for correct and fast responses were orthogonally manipulated across four task blocks. Results show that reward availability affected a prefrontal reward P200 and a centro-parietal P300 ERP. Moreover, a fronto-central ERP was affected by both reward and probability manipulations. These results suggest that reward and probability are partially separately processed in the cortex. Furthermore, reward and probability information are integrated around $300 \mathrm{~ms}$ after presentation of the cue and possibly processed via a shared underlying cortical mechanism that may act to reduce uncertainty or to prepare for action. In a follow-up study we investigated the role of dopamine (and noradrenaline) in either of these processes by employing a within-subjects haloperidol/clonidine/ placebo cross-over design $(n=24)$ with the same cued Go/NoGo paradigm.

Trial registration

The Netherlands National Trial Register (NTR) $(C C=4493)$.

A38

Optimizing the earliest memory stages: a role for acetylcholine and serotonin?

Anke Sambeth (anke.sambeth@maastrichtuniversity.nl)

Neuropsychology and Psychopharmacology, Maastricht University, Maastricht, the Netherlands

Neuropsychiatric Electrophysiology 2016, 2(Suppl 1):A38

Acetylcholine and serotonin both play an important role in encoding and consolidation of memories. However, it has also been suggested that these two neuromodulators take part, and might even interact, in processes initiated before conscious encoding even takes place. Sensory memory and novelty detection, two processes related to reduction of surprise, are part of those early stages. They can be measured with the mismatch negativity (MMN) and P3a components of the event-related potential, respectively. In a series of experiments, we examined whether cholinergic and serotonergic manipulations affect MMN and P3a components during a novelty oddball task. In this task, frequent standard stimuli were interspersed with infrequent deviant and infrequent novel stimuli at a pace of one stimulus presentation per second. Biperiden, a cholinergic agonist, and rivastigmine, a cholinesterase inhibitor, did not affect MMN amplitude. Acute tryptophan depletion, a method to reduce serotonin in the brain, and citalopram, a selective serotonin re-uptake inhibitor, were 
also unable to affect the MMN. No significant interactions between treatments were found related to the MMN. Cholinergic treatments did, however, affect the P3a amplitude: P3a was decreased after Biperiden intake and increased after Rivastigmine. The serotonergic manipulations did not affect P3a amplitude, neither were interactions found between treatments. Our results thus show that, although the cholinergic and serotonergic systems do not seem to play a role in sensory memory, acetylcholine's role in novelty detection, and thus in handling surprise, is evident.

\section{A39}

Processing of the mismatch negativity under the LSD state Christopher Timmermann 1, 2, Mendel Kaelen', Eduardo Schenberg ${ }^{1}$, Amanda Feilding ${ }^{3}$, Robert Leech ${ }^{2}$, David Nutt ${ }^{1}$, Robin Carhart-Harris ${ }^{1}$, Suresh Muthukumaraswamy ${ }^{4}$

${ }^{1}$ Centre for Neuropsychopharmacology, Department of Medicine, Imperial College London, London, UK; ${ }^{2}$ Computational, Cognitive and Clinical Neuroscience Laboratory, Department of Medicine, Imperial College London, London, UK; ${ }^{3}$ The Beckley Foundation, Oxford, UK; ${ }^{4}$ CUBRIC, School of Psychology, Cardiff University, Cardiff, UK Neuropsychiatric Electrophysiology 2016, 2(Suppl 1):A39

\section{Background}

Lysergic acid diethylamide (LSD) is a classic psychedelic drug and serotonin $2 \mathrm{~A}$ receptor agonist. A common feature of the LSD state is its capability to provide an experience of modulating the salience of external events. The Mismatch Negativity (MMN) is an event-related potential/field (ERP/ERF), that indexes expectation disruption (or 'surprise') mechanisms, which have been shown to be modulated in patients with disorders of consciousness, as well as in schizophrenia and following ketamine administration. In this study the MMN paradigm was used to assess expectation and surprise mechanisms under LSD and placebo conditions in healthy participants.

Methods

A balanced order, within subject design was used for the study. 20 Healthy volunteers underwent MEG recordings following intravenous administration of LSD (75 mcg IV) and placebo at least 2 weeks apart. Participants were presented with auditory stimuli consisting of oddball and standard tones while resting inside the MEG scanner. Following preprocessing and averaging, the resulting eventrelated fields (ERF) were converted into scalp-map images and smoothed for statistical analysis corresponding to four conditions: auditory stimuli of standard tones under LSD (1) and placebo (2) and deviant tones under LSD (3) and placebo (4). The ERFs were entered into a within-subject analysis of variance with 2 main factors: 'drug' (LSD and placebo) and 'expectation disruption' (standard and deviant).

Results

An interaction effect between 'expectation disruption' and 'drug' factors was found in a right lateralized cluster in the scalp. Post hoc analyses within this ROI, reveal significant differences in the processing of standard tones between placebo and LSD conditions as well as deviant tones. Within the placebo condition the difference between standards and deviants was significant, while it wasn't following LSD administration.

\section{Conclusions}

Results indicate a reduction of activity related to the processing of novel stimuli, while showing that the surprise response was increased under the LSD condition in large areas of the scalp for familiar stimuli. These findings may inform how salience mechanisms may be disrupted under LSD and is consistent with reports of "increased novelty" to familiar stimuli in the LSD state. Mechanisms underlying this modulation may be accounted by modulation of prediction error in the psychedelic state.

This research received financial and intellectual support from the Beckley Foundation and was conducted as part of a wider BeckleyImperial research programme.
A40

The effects of psilocybin on human EEG, comparison with animal models

Tomas Palenicek ${ }^{1,2}$, Filip Tyls ${ }^{1,2}$, Michaela Viktorinova', Anna Bravermanova ${ }^{1,2}$, Renata Androvicova', Vaclava Sedlamyerova', Vladimir Krajca', Martin Brunovsky ${ }^{1}$

${ }^{1}$ National Institute of Mental Health, Klecany, Czech Republic; ${ }^{2}$ Third

Faculty of Medicine, Charles University, Prague, Czech Republic

Neuropsychiatric Electrophysiology 2016, 2(Suppl 1):A40

\section{Objectives}

Psilocin, the active metabolite of psilocybin, is a classical psychedelic tryptamine acting as an agonist at serotonin $5-\mathrm{HT} 2 \mathrm{~A} / \mathrm{C}$ and $5-\mathrm{HT} 1 \mathrm{~A}$ receptors. It has been used extensively to model psychosis in humans as well as in animals, and during the last decade given more attention as a potential antidepressant and anxiolytic drug. Recent studies in healthy volunteers have shown that psilocybin leads to a global desynchronization of brain activity and disconnection of the main brain networks. The purpose of the current study is to compare the translational validity of psilocybin/psilocin effects on EEG activity and connectivity between healthy volunteers and rats.

Methods

For the human study, a 19 channel EEG of a standard 10/20 system was recorded with BioSDA09 amplifier (M\&l). Subjects (M10/F10) were administered $0.26 \mathrm{mg} / \mathrm{kg}$ of psilocybin or placebo orally in a doubleblinded, crossover manner. EEG was recorded before and at 50, 90, 180 and 240 min after drug administration. In the animal study, psilocin was administered to male Wistar rats $(n=10)$ subcutaneously in dose of $4 \mathrm{mg} / \mathrm{kg}$. Multichannel EEG with 12 cortical (6 homolateral pairs) electrodes at frontal, parietal and temporal regions were recorded for $10 \mathrm{~min}$ before and $90 \mathrm{~min}$ after dosing using BrainScope EADS 221 amplifier (M\&l). EEG in animals was co-recorded with behavioural activity and epochs of inactivity were then subjected for further processing. Data was pre-processed using Brainvision and WaveFinder software followed by further power spectral and coherence analyses using Neuroguide software. Source localization of EEG activity was analysed by LORETA, 3D brain mapping in animals was performed using an inhouse developed Matlab tool.

Results

Psilocybin induced an absolute as well as relative alpha power decrease in occipital regions, while beta and gamma power increases in frontotemporal areas in humans. The effects were most robust 50 min after drug administration. Source localization by LORETA confirmed the localization of EEG changes. However, it is of note that the gamma power cannot be distinguished from artificial motor activations. EEG Coherence was mainly decreased in theta, alpha and beta bands, with some increases observed in beta and gamma bands. In rats there was a global power decrease in absolute power however relative power showed partly similar profile to humans with theta power decrease and beta and gamma power increase. Furthermore, there was a global decrease of coherence in rats following drug administration.

\section{Conclusions}

The effects of psilocin/psilocybin resulted in similar direction of EEG changes in both humans and rats with disconnection (decreased coherence) being the most stable phenomenon observed, indicating good translational validity.

This study was supported by the projects IGA MHCR NT/13897, ED2.1.00/03.0078, LO1611/NPU I, MH CZ - DRO (NIMH-CZ, 00023752) and PRVOUK P34.

\section{A41}

Salvinorin-A induces a unique pattern of neurophysiological

effects in humans characterized by alpha suppression and widespread increases in cortical delta activity

M Valle', A E Maqueda', S Romero², M A Mañanas², S Barker', J Riba

${ }^{1}$ Hospital de la Santa Creu i Sant Pau, Barcelona, Spain; ${ }^{2}$ Universitat

Politècnica de Catalunya (UPC), Barcelona, Spain; ${ }^{3}$ Louisiana State

University, Baton Rouge, LA, USA.

Correspondence: $M$ Valle

Neuropsychiatric Electrophysiology 2016, 2(Suppl 1):A41 


\section{Background}

Salvinorin-A (SA) is a potent perception-modifying drug found in the leaves of the plant Salvia divinorum. Unlike $5-\mathrm{HT}_{2 \mathrm{~A}}$ agonists such as DMT, LSD and psilocybin, SA is a selective kappa-opioid receptor (KOR) agonist. Its pattern of effects in humans also shows important differences with that of the classical psychedelics. While subjects also experience intense visual and auditory phenomena, SA completely blocks external sensory perception, and leads to a characteristic total loss of contact with external reality. Here we investigated the neurophysiological correlates of SA effects in humans.

Methods

We measured spontaneous brain oscillations (EEG) in 24 healthy volunteers, before and after the administration of $1 \mathrm{mg}$ vaporized SA. We recorded the EEG from 19 scalp leads and we calculated drug-induced energy changes in ten frequency bands between 1.3 and $40 \mathrm{~Hz}$. Additionally, we computed the changes in the intracerebral current density distribution associated with the voltage values recorded at the scalp.

Results

SA administration led to rapid and significant changes in brain oscillations that coincided with maximum drug levels in plasma. SA suppressed the alpha rhythm $(7.5-13 \mathrm{~Hz})$ and markedly increased slow delta activity $(1.3-3.5 \mathrm{~Hz})$. Less prominent effects included increases in the theta $(3.5-7.5 \mathrm{~Hz})$ and low gamma (35$40 \mathrm{~Hz}$ ) bands. Alpha decreases were localized over parietooccipital regions, including the posterior cingulate cortex and visual areas. Delta increases were observed over most of the brain, with the maximum located over auditory and visual cortex in the left temporal lobe. Theta increases were found over left temporal and frontal areas. Finally, gamma increases were restricted to visual areas in the occipital cortex.

Conclusion

These results show a unique pattern of neurophysiological effects for SA in humans. While it shares with serotonergic psychedelics the alpha-suppressing action, its main neurophysiological signature is an atypical enhancement of slow delta activity. These differences may explain the marked differences in subjective effects between SA and $5-\mathrm{HT}_{2 \mathrm{~A}}$ agonists.

This research was supported by Grant PI12/02758 from the Spanish government

\section{A42}

QEEG signatures predicting antidepressant response to ketamine

Martin Brunovsky ${ }^{1,2}$, Jiri Horacek ${ }^{1,2}$, Tomas Palenicek ${ }^{1,2}$, Peter Sos ${ }^{1}$, Filip Tyls $^{1,2}$, Michaela Viktorinova ${ }^{1,2}$, Cyril Höschl ${ }^{1,2}$

${ }^{1}$ National Institute of Mental Health, Klecany, Czech Republic; ${ }^{2}$ Third Faculty of Medicine, Charles University, Prague, Czech Republic

Correspondence: Martin Brunovsky (martin.brunovsky@nudz.cz) Neuropsychiatric Electrophysiology 2016, 2(Suppl 1):A42

\section{Objective}

Treatment-resistant depression (TRD) is a disabling disorder that negatively impact patient's morbidity and mortality and constitutes a major challenge for current psychopharmacology. The discovery of rapid-acting antidepressive action of ketamine has motivated studies aiming to reveal the molecular mechanism of this effect and to enable the clinical application of similarly rapid-acting antidepressants. In our two studies, the time-course of effects of ketamine was assessed in treatment-resistant depressive patients by QEEG to elucidate changes associated with treatment effect and to assess potential predictors of treatment response.

Methods

The pool analysis was completed from data of two double-blind, cross-over, placebo-controlled studies, assessing the effect of single infusion of ketamine $(0.54 \mathrm{mg} / \mathrm{kg}$ within $30 \mathrm{~min})$ in altogether 50 inpatients with major depressive disorder. EEG data were analysed during the infusion (10 $\mathrm{min}$ and $30 \mathrm{~min}$ ) and $24 \mathrm{~h}$ after ketamine administration using exact low-resolution electromagnetic tomography (eLORETA). Response to treatment was defined as a $\geq 50 \%$ reduction of MADRS score.
Results

Ketamine induced immediate (10 $\mathrm{min}$ and $30 \mathrm{~min}$ ) decrease of parietooccipital sources of alpha- 1 and alpha-2 activities and an increase of gamma-sources in all subjects. Responders to medication were characterized by excess of mediofrontal delta and theta sources in comparison to non-responders. Moreover, only the responders showed significant changes that persisted $24 \mathrm{~h}$ after infusion, while no significant changes were observed in non-responders. Among the clinical variables we have found a significant correlation between the BPRS score during ketamine infusion and MADRS score at day 7 , and the intensity of psychotomimetic symptoms during infusion seems to be the strongest clinical predictor of antidepressive effect of ketamine. Regarding the QEEG parameters, the patients with better responses showed higher pre-treatment theta activity in mediofrontal areas and in the rostral anterior cingulate. Better response to ketamine was also connected with higher pre-treatment lagged phase synchronization (i.e. higher connectivity) between anterior cingulate and mediofrontal cortex at theta and alpha- 1 frequency bands.

Conclusion

Our results suggest that an acute increase of mediofrontal cortical sources of theta and delta activities after ketamine infusion could be potential biomarkers to differentiate responders and non-responders to ketamine. Higher pre-treatment theta activity in mediofrontal areas together with higher lagged phase synchronization between anterior cingulate and mediofrontal cortex at theta and alpha- 1 frequency bands could serve as predictors of treatment response to ketamine. Moreover, the antidepressive effect of ketamine seems to be undoubtedly connected with patient's psychotomimetic experience.

Supported by grants AZV MZCR 15-33250A and by the project PRVOUK P34.

\section{Oral presentations}

A43

Nonlinearity of the visual system assessed by cross-frequency

phase coupling

Matthiis JL Perenboom ${ }^{1}$,Yuan Yang ${ }^{3}$, Frans CT van der Helm³ ${ }^{3}$, Michel D

Ferrari ${ }^{1}$, Alfred C Schouten ${ }^{3,4}$, Else A Tolner ${ }^{1,2}$

'Department of Neurology, Leiden University Medical Centre, Leiden,

The Netherlands; ${ }^{2}$ Department of Human Genetics, Leiden University

Medical Centre, Leiden, The Netherlands; ${ }^{3}$ Department of Biomechanical

Engineering, Delft University of Technology, Delft, The Netherlands;

${ }^{4}$ MIRA Institute for Biomedical Technology and Technical Medicine,

University of Twente, Enschede, The Netherlands

Correspondence: Matthijs JL Perenboom (M.J.L.Perenboom@lumc.nl)

Neuropsychiatric Electrophysiology 2016, 2(Suppl 1):A43

\section{Background}

Processing of visual input by the brain is a highly nonlinear operation, involving complex interactions among neuronal networks. Nonlinear visual system activity includes harmonic interactions, thought to reflect resonance of neural processing, whereas intermodulation, being the contribution of multiple input frequencies to one output frequency, relates to functional integration [1]. Using a sumof-sinusoid signal as visual input [2], it is possible to elicit a richer class of nonlinear responses than the classic pulse train stimulus, thereby providing a more complete description of nonlinearity. Here, we will use nonlinear EEG analyses to quantify higher-order nonlinearities in visual processing.

Methods

Ten healthy participants were subjected to bi-sinusoidal light stimulation of 13 and $23 \mathrm{~Hz}$ for $3201 \mathrm{~s}$-epochs, while scalp EEG (8 electrodes) was recorded at the occipital, parietal and frontal lobes. The frequencies of light stimulus were chosen to guarantee no overlap of their harmonic and intermodulation frequencies for different orders of nonlinearity. Nonlinear interactions and time delay from stimulus to cortex were analyzed in the frequency domain using novel phase synchronization measures [3] and amplitude spectrum.

Results

Higher harmonic and intermodulation interactions were detected between visual input and cortical responses. First to fourth order 
phase coupling interactions were enhanced in the visual cortex compared to parietal and frontal responses. Spectral amplitude differences were less pronounced between cortical regions. Time delay estimation showed a delay between light stimulus and visual cortex of $118 \pm 21 \mathrm{~ms}$, significantly higher than the delay between stimulus and frontal or parietal lobes.

Discussion

This study demonstrates the potential of using sum-of-sinusoid light stimulation and quantitative nonlinear EEG analysis to identify higher-order nonlinear dynamics of visual processing. We foresee that application of the described frequency interaction analyses can further our insight in the nonlinear dynamics of visual processing not only in healthy subjects, but also with respect to the pathophysiology of neurological diseases with visual manifestations that relate to cortical hyperexcitability, like migraine and epilepsy.

\section{References}

1. Friston KJ. Book review: Brain function, nonlinear coupling, and neuronal transients. Neuroscientist. 2001 Oct 1;7(5):406-18.

2. Victor J, Shapley R. A method of nonlinear analysis in the frequency domain. Biophys J. 1980 Mar;29(3):459.

3. Yang Y, Solis-Escalante T, Yao J, Daffertshofer A, Schouten AC, van der Helm FC. A General Approach for Quantifying Nonlinear Connectivity in the Nervous System Based on Phase Coupling. Int J Neural Syst. 2016 Feb;26(01):1550031.

\section{A44}

Whole-brain time-frequency analysis of event-related potentials for the assessment of pharmacodynamic effects in the human brain Roman Rosipal ${ }^{1,3}$, Leonardo Jose Trejo ${ }^{1}$, John Wallerius', Ross Apparies', Barbora Cimrova ${ }^{1,3,4}$, James Miller $^{2}$

${ }^{1}$ Pacific Development and Technology, LLC, Palo Alto, CA 94303, USA;

${ }^{2}$ Neuro Assessment Systems, Inc., Littleton, CO 80123, USA; ${ }^{3}$ Slovak Academy of Sciences, Bratislava, Slovakia; ${ }^{4}$ Comenius University,

Bratislava, Slovakia

Correspondence: Roman Rosipal (rrosipal@pacdel.com)

Neuropsychiatric Electrophysiology 2016, 2(Suppl 1):A44

We are developing Whole Brain Time-Frequency (WBTF) analysis as a new physiological biomarker for clinical trials of pharmacodynamics of novel drugs. WBTF analysis expands the power of event-related potential (ERP) assessment by using wavelets to measure both evoked (phase-locked) and induced (non-phase-locked) activity. Unlike traditional ERP measures, which are indexed by specific electrodes and peak latencies, WBTF analysis measures integrated change in brain responses across time, frequency and space to infer whether a drug has a significant effect. WBTF analysis also uses permutation tests and multiple comparison corrections to identify important within-subject changes between conditions and rule out differences arising from recording noise, artifacts or random variability.

The specific aim of this study was to assess the sensitivity and specificity of WBTF analysis to drug effects that are typically measured with ERP amplitudes and latencies. We simulated effects of dose-related changes in N1-P2-P3 ERP components and $40-\mathrm{Hz}$ induced gamma bursts at 24 electrodes. Simulations included a range of amplitude effects, latency effects and signal-to-noise ratios, serving to define the sensitivity and specificity of WBTF analysis to ERP differences.

The simulations allowed us to optimize parameters for WBTF analysis, including choice of analyzing wavelets, energy normalization, baseline correction, measures of evoked and induced activity, and method of testing significant differences. We found that WBTF analysis reliably detects small differences in evoked activity (on the order of $10 \%$ ) in realistic noise and background EEG conditions. We found similar detectability of small differences in induced $40-\mathrm{Hz}$ gamma bursts.

It is the goal of the further studies to investigate the clinical relevance of these observed differences using WBTF analysis, and to relate the evoked and induced components ERP differences to mechanisms of drug action. Currently we are applying WBTF analysis to data from three Phase 1 clinical trials of novel compounds for schizophrenia in both healthy controls and schizophrenia patients.
A45

Dysregulation of hyperpolarization-activated inward cation current $\left(I_{h}\right)$ affects thalamocortical oscillations: the role of the auxiliary subunit TRIP8b on HCN channel function in thalamic and cortical neurons

Mehrnoush Zobeiri', Rahul Chaudhary', Annika Lütjohann'1, Patrick Meuth', Hans-Christian Pape', Dane M Chetkovich², Gilles van Luijtelaar ${ }^{3}$, Thomas Budde

${ }^{1}$ Institute für Physiologie I, Westfälische Wilhelms-Universität, Münster, Germany; ${ }^{2}$ Davee Department of Neurology and Clinical Neurosciences and Department of Physiology, Northwestern University, Chicago, IL, USA; ${ }^{3}$ Donders Centre for Cognition, Radboud University Nijmegen, Nijmegen, The Netherlands

Neuropsychiatric Electrophysiology 2016, 2(Suppl 1):A45

\section{Background}

The family of hyperpolarization-activated cyclic nucleotide-gated cation (HCN) channels consisiting four different isoforms (HCN1-4) have a major role in controlling neuronal excitability and generation of rhythmic oscillatory activity in individual neurons and neuronal networks [1, 2]. These channels activate in response to hyperpolarizing potentials negative to -50 to $-60 \mathrm{mV}$ and depolarize the resting membrane potential. HCN channels are regulated by small molecules like cyclic nucleotides and different accessory proteins. TRIP8b is a brain-specific accessory subunit of $\mathrm{HCN}$ channels which controls the gating, surface expression and trafficking of different $\mathrm{HCN}$ channels subunits in many regions of brain [3-5]. The role of this protein for $I_{h}$ characteristics in thalamic and cortical neurons and the functional consequences of TRIP8b dysregulation for thalamocortical oscillations however is not yet fully understood. The present study aimed at providing a better understanding of the functional role of TRIP8b in the thalamocortical system and shedding some light on possible dysfunctional aspects by combining in vitro and in vivo electrophysiological approaches.

In this study, $I_{h}$ was measured in whole cell patch clamp recordings from thalamocortical (TC) neurons of different thalamic nuclei, as well as pyramidal neurons in layer $\mathrm{V}$ and $\mathrm{VI}$ of the somatosensory cortex of TRIP8b-deficient (TRIP8b ${ }^{-1-}$ ) and control (C57BI/6 J) mice (p15 p90). Effects of TRIP8b-dependent dysregulation of $I_{h}$ on thalamocortical oscillations was monitored by local field potential (LFP) recordings from the ventral-posterior medial complex of the thalamus (VPM) and somatosensory cortex ( $p 90-p 120)$, regions which are known to be involved in generation of normal and also pathological thalamocortical oscillations.

Results

Characterization of $I_{h}$ in the thalamocortical system in the absence of the auxiliary subunit TRIP8b showed a significant decrease in $I_{h}$ density and changes in intrinsic properties and firing patterns of TC and cortical pyramidal neurons. These changes were accompanied by an increase in CAMP sensitivity in TC neurons. Dysregulation of $I_{h}$ in the thalamocortical system of $\mathrm{TRIP}^{-1-}$ mice was associated with altered thalamocortical oscillations revealing a significant increase in slow oscillations in the delta frequency range $(0.5-4 \mathrm{~Hz})$ during episodes of active-wakefulness.

Conclusion

The results of our study point to the importance of TRIP8b, as a brain-specific auxiliary subunit of HCN channels, in regulation of cell and network oscillations. It was demonstrated here that the presence of TRIP8b is necessary for modulation of thalamocortical delta oscillations due to its direct effect on $\mathrm{HCN}$ channels protein expression in the thalamocortical system.

\section{References}

1. Wahl-Schott, C. \& Biel, M. HCN channels: Structure, cellular regulation and physiological function. Cell. Mol. life Sci. 66, 470-494 (2009).

2. Biel, M., Wahl-Schott, C., Michalakis, S. \& Zong, X. Hyperpolarizationactivated cation channels: from genes to function. Physiol. Rev. 89, 847-85 (2009).

3. Bina Santoro, Rebecca A. Piskorowski, Phillip Pian, Lei Hu, Haiying Liu and S. A. S. TRIP8b splice variants form a family of auxiliary subunits that regulate gating and trafficking of HCN channels in the brain. Neuron 81, 8715-8723 (2009). 
4. Lewis, A. S. et al. Alternatively spliced isoforms of TRIP8b differentially control h channel trafficking and function. J. Neurosci. 29, 6250-6265 (2009).

5. Pan, Y. et al. TRIP8b is required for maximal expression of HCN1 in the mouse retina. PLoS One 9, e85850 (2014).

6. Piskorowski, R., Santoro, B. \& Siegelbaum, S. A. TRIP8b splice forms act in concert to regulate the localization and expression of HCN1 channels in CA1 pyramidal neurons. Neuron 70, 495-509 (2011).

7. Santoro, B. et al. Article TRIP8b Splice Variants Form a Family of Auxiliary Subunits that Regulate Gating and Trafficking of HCN Channels in the Brain. Neuron 62, 802-813 (2009).

8. Heuermann, R. J. et al. Reduction of thalamic and cortical Ih by deletion of TRIP8b produces a mouse model of human absence epilepsy. Neurobiol. Dis. 85, 81-92 (2016).

\section{A46}

Advanced EEG imaging of neuronal network interactions during spatial working memory performance in rats: paving the road for pharmacological assessments

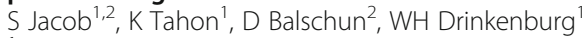

${ }^{1}$ Department of Neuroscience, Janssen Research \& Development, a Division of Janssen Pharmaceutica NV, 2340, Beerse, Belgium;

2.Laboratory of Biological Psychology, University of Leuven, 3000, Leuven,Belgium

Correspondence: S Jacob (sjacob6@|TS.JNJ.com)

Neuropsychiatric Electrophysiology 2016, 2(Suppl 1):A46

Cognitive processes are based on the coordination of interactions of populations of neurons that are distributed within and across different specialized brain areas. Accumulating evidence suggests that neuronal oscillations play a pivotal role in driving brain communication. This communication is affected in many neurodegenerative diseases. Accordingly, understanding network interactions during cognitive activity is crucial for a better comprehension of neurodegenerative consequences for cognitive functioning as well as for assessing the efficacy of novel pharmacological treatments. The purpose of the present study was to evaluate putative EEG-based biomarkers during the trial-unique delayed nonmatching-to-location (TUNL) task in rats. The task assesses memory for location across different delays and spatial separations in a computer-automated touchscreen set-up. Once EEG-instrumented rats reached performance criteria (80\% accuracy in an $8 \mathrm{~s}$ delay for two consecutive days), brain activity in the CA1 region of the hippocampus, medial prefrontal cortex, and retrospenial cortex was monitored during two consecutive TUNL sessions using an $8 \mathrm{~s}$ and a $16 \mathrm{~s}$ delay. Time frequency-based analysis of EEG readouts was used to investigate neuronal connectivity during the different delays comparing correct vs. incorrect trials. In particular, cross-frequency coupling (CFC, when the phase of a low frequency oscillation drives the amplitude of the coupled higher frequency oscillation) was analyzed as this has been suggested a possible mechanism facilitating working memory. It was hypothesized that functional connectivity during the delay will be reduced during incorrect trials compared to correct ones for both delays and separations. Behavioral results confirmed that accuracy was higher in the larger separation for the 8 s delay $(M=84.29 \%, 95 \%$ Cl $[81.03,87.55])$ compared with smaller separation for the same delay $(M=74.99 \%, 95 \%-C l[69.89,80.09])$. Furthermore, the $16 \mathrm{~s}$ appeared to be more challenging as accuracy was reduced for both large $(M=63.05 \%, 95 \%-C l[55.95,70.15])$ and small separation $(M=$ $60.41 \%, 95 \%-C l[55.57,65.25])$ compared with the 8 s delay. Interestingly, results from the CFC during the delay support our hypothesis as $8 \mathrm{~Hz}$ frequency modulation of $90 \mathrm{~Hz}$ amplitude in the medial prefrontal cortex showed a more rapid decrease in CFC during the delay for the incorrect trials in both delays and separations compared to the correct trials. Overall, results identified the critical role of neuronal oscillations and connectivity for working memory in the TUNL task. This study reinforces the strength of combining multiple approaches to further understand cognitive processes and assessment of pharmacological treatments.
A47

EEG functional connectivity of Brodmann area 24 in obsessivecompulsive disorder

J Koprivova', E Saifutdinova', T Nekovarova', M Raszka', J Prasko²

${ }^{1}$ National Institute of Mental Health, Klecany, Czech Republic; ${ }^{2}$ Faculty of Medicine and Dentistry,Palacky University Olomouc, University Hospital,

Olomouc, Czech Republic

Correspondence: J Koprivova (jana.koprivova@nudz.cz)

Neuropsychiatric Electrophysiology 2016, 2(Suppl 1):A47

\section{Background}

A growing body of evidence have challenged the traditional orbitofrontostriatal hypothesis and suggested that dysregulation of widespread brain networks may underlie the OCD disorder. In last decades, the increasing attention of neuroscience research has been payed to large-scale network organization of the brain. Three prominent networks were identified: "Central Executive Network", "Default Mode Network" and "Salience Network", responsible for synchronization of anticorrelated activity of DMN and CEN. Several studies have stressed the role of the dorsal anterior cingulate cortex, a core structure within the salience network, in OCD pathophysiology. Our two previous studies also revealed abnormal EEG activity in this structure (Brodmann area, BA 24), however little is known about its EEG functional connectivity in OCD. Based on our previous findings, we tested functional connectivity between EEG sources in BA 24 and rest of the brain in the group of OCD patients and in heathy controls.

Methods

96 in-patients diagnosed with OCD and 95 healthy controls matched for age and sex were included in the study. All subjects were righthanded. 27 OCD patients were drug-free and 69 were medicated with SSRIs. All subjects underwent 19-channel resting-state EEG examination. Functional connectivity was analysed in LORETA-KEY software. We assessed connectivity between centroid of BA 24 and centroids of all the other Brodmann areas as defined in the LORETA-KEY software. Lagged nonlinear connectivity was computed in eight frequency bands: delta $(1.5-6 \mathrm{~Hz})$, theta $(6.5-8 \mathrm{~Hz})$, alpha $1(8.5-10 \mathrm{~Hz})$, alpha $2(10.5-12 \mathrm{~Hz})$, beta1 $(12.5-18 \mathrm{~Hz})$, beta $2(18.5-21 \mathrm{~Hz})$, beta 3 $(21.5-30 \mathrm{~Hz})$ and gamma $(30.5-44.0 \mathrm{~Hz})$. Groups were compared using tstatistics and permutation testing to correct for multiple comparisons. Results

Drug-free and SSRIs medicated patients did not differ from each other in functional connectivity and therefore they were further tested as a unitary group. Compared with controls, OCD patients had higher lagged nonlinear functional connectivity between BA 24 and BA 5 and BA 7 in the beta 3 as well as in the gamma frequency band $(p<0.05)$. In the gamma band the results were significant only for the left BA 5 and 7, however connectivities in the right hemisphere were close to threshold.

\section{Conclusion}

We hypothesize that an aberrant synchronization between default mode and central executive network related to the aberrant activity of the salience network may underlie symptoms of OCD.

This work was supported by the projects ED2.1.00/03.0078, L01611 and MH CZ - DRO 00023752.

\section{A48}

Cortical network reorganization in mild and prodromal Alzheimer disease: graph theory approach on resting state EEG recordings L Bonanni $^{1}$, R Franciotti ${ }^{1}$, NW Falasca ${ }^{2}$, F Nobili $^{3}$, D Arnaldi $^{3}$, M Onofrj $^{1}$ ${ }^{1}$ Department of Neuroscience, Imaging and Clinical Science, and Aging Research Centre, G. d'Annunzio University, Chieti, 66013, Italy; ${ }^{2}$ BIND Behavioral Imaging and Neural Dynamics Center, University of ChietiPescara, Chieti, 66013, Italy; ${ }^{3}$ Department of Neuroscience (DINOGMI), Clinical Neurology, University of Genoa and IRCCS AOU San Martino-IST, Genoa, 16126, Italy

Correspondence: L Bonanni (I.bonanni@unich.it) Neuropsychiatric Electrophysiology 2016, 2(Suppl 1):A48 


\section{Background}

Alzheimer's disease (AD) has a long preclinical period in the absence of overt symptoms in which the process progresses until it crosses a threshold to clinically recognizable dysfunction [1]. Graph theoretical analysis of cerebral networks has been implemented in AD challenging the classical concept of neurological disorders being either 'local' or 'global', and have pointed to the overload and failure of hubs as a possible final common pathway in neurological disorders. Previous EEG studies on the comparison between $A D$ and control subjects reported divergent results [2]. An intermediate trend was found in subjects with mild cognitive impairment $(\mathrm{MCl})$ with respect to $A D$ and control subjects [3]. The aim of the study was to asses by means of graph theory analysis if $A D$ patients with mild dementia could show a different cortical organization from age matched control subjects and if these possible differences could be already present at the stage of $\mathrm{MCl}$.

\section{Results}

The main finding of the present study is that network reorganization is evident in $A D$ since the prodromal stage (AD-MCI). Specifically, AD$\mathrm{MCl}$ and $A D$ showed a lower number of links among nodes than control group $(p=0.0007)$. Both inward and outward links among nodes and brain areas with a high level of functional connectivity (so-called hubs) were found to be reduced in both $A D-M C l$ and $A D$ patients. Hubs in the parietal areas (P3, P4, and Pz) showed lower number of links in $\mathrm{AD}-\mathrm{MCl}$ and $\mathrm{AD}$ than control group. Temporal nodes showed lower clustering coefficient and local efficiency in patients than control group. Significant differences between AD-MCI and $A D$ were found in the right occipital node. Indeed, the clustering coefficient and the local efficiency was reduced in $A D$ compared with $\mathrm{AD}-\mathrm{MCl}$ in $\mathrm{O} 2(\mathrm{p}<0.05)$

\section{Conclusions}

Our results suggest that brain network functional alterations mainly involved the temporal nodes in prodromal stage of $A D$, whereas brain dynamic changed in the posterior areas with disease progression to overt AD dementia.

The functional disconnection between temporal and parietooccipital areas could be related to medial temporal lobe atrophy which is a characteristic neuropathological change in the early stage of $A D$ [4].

\section{References}

1. Braak H, Thal DR, Ghebremedhin E, Del Tredici K. Stages of the pathologic process in Alzheimer disease: age categories from 1 to 100 years. J Neuropathol Exp Neurol. 2011;70:960-9.

2. de Haan W, Pijnenburg YA, Strijers RL, van der Made Y, van der Flier WM, Scheltens $\mathrm{P}$, Stam CJ. Functional neural network analysis in frontotemporal dementia and Alzheimer's disease using EEG and graph theory. BMC Neurosci. 2009;10:101.

3. Vecchio F, Miraglia F, Quaranta D, Granata G, Romanello R, Marra C, Bramanti P, Rossini PM. Cortical connectivity and memory performance in cognitive decline: A study via graph theory from EEG data. Neuroscience. 2016;316:143-50.

4. Rusinek H, Endo Y, De Santi S, Frid D, Tsui WH, Segal S, Convit A, de Leon MJ. Atrophy rate in medial temporal lobe during progression of Alzheimer disease. Neurology 2004;64:2354-9.

\section{A49}

Sleep disturbances in obsessive-compulsive disorder: association with response to repetitive transcranial magnetic stimulation (rTMS)

Lana Donse', Alexander T Sack ${ }^{4,5}$, Paul B Fitzgerald ${ }^{6}$, Martijn Arns ${ }^{1,2,3}$ ${ }^{1}$ Research Institute Brainclinics, Nijmegen, The Netherlands;

${ }^{2}$ Department of Experimental Psychology, Utrecht University, Utrecht, The Netherlands; ${ }^{3}$ neuroCare Group, Munich, Germany; ${ }^{4}$ Department of Cognitive Neuroscience, Maastricht University, Maastricht, The Netherlands; ${ }^{5}$ Maastricht Brain Imaging Center, Maastricht, The Netherlands; ${ }^{6}$ Monash Alfred Psychiatry Research Centre, the Alfred and Monash University, Central Clinical School, Victoria, Australia Correspondence: Lana Donse (lana@brainclinics.com)

Neuropsychiatric Electrophysiology 2016, 2(Suppl 1):A49

\section{Background}

Obsessive-compulsive disorder (OCD) is a debilitating disorder with a substantial portion of patients not responding to first-line treatment. Repetitive transcranial magnetic stimulation (rTMS) is an effective augmentation strategy for treatment-refractory patients, but as the response rate is approximately $35 \%$ [1], it is important to identify predictors of rTMS response. Emerging evidence indicates a relatively high prevalence of sleep disturbances in OCD patients, in particular circadian rhythm sleep disorder (CRSD) [2]. It is therefore proposed that sleep disturbances may affect treatment efficacy.

Methods

In this open-label study, 22 OCD patients received at least 10 sessions of rTMS treatment targeted at the supplementary motor area combined with psychotherapy. They were compared to a matched control group of healthy subjects to examine sleep disturbance. Treatment outcome was measured by monitoring obsessive-compulsive as well as depressive symptoms each fifth session using the YBOCS and BDI, respectively. Treatment response was defined as a reduction $>35 \%$ on the YBOCS. Sleep disturbances were measured by means of self-report (PSQI, HSDQ) and actigraphy. Treatment response prediction models were based on subjective and objective measures of CRSD and insomnia. Results

OCD patients showed a higher rate of sleep disturbances than controls. The OCD group consisted of 12 responders and 10 non-responders. Responders showed a significantly larger reduction in both obsessivecompulsive and depressive symptoms, while no difference in baseline severity existed. Sleep disturbances, on the contrary, were more severe in non-responders than responders. Furthermore, a predictive model based on CRSD could accurately predict treatment response with $83 \%$ sensitivity and $63 \%$ specificity, whereas the insomnia model could not. Conclusions

Sleep disturbances in OCD can significantly predict rTMS treatment response, in particular CRSD. Therefore, CRSD may serve as a biomarker for different subtypes of OCD that correspond with response to specific treatment approaches.

\section{Competing interests}

PBF is supported by a NHMRC Practitioner Fellowship (1078567). PBF has received equipment for research from MagVenture A/S, Medtronic Ltd, Cervel Neurotech and Brainsway Ltd and funding for research from Neuronetics and Cervel Neurotech. He is on the scientific advisory board for Bionomics Ltd. MA reports research grants, options/shares from Brain Resource Ltd. (Sydney, Australia) and neuroCare group and he is also a co-inventor on 4 patent applications (A61B5/0402; US2007/0299323, A1; WO2010/139361 A1) related to $\mathrm{EEG}$, neuromodulation and psychophysiology, but does not own these nor receives any proceeds related to these patents.

\section{References}

1. Berlim, M. T., Neufeld, M. H., Van den Eynde, F. Repetitive transcranial magnetic stimulation (rTMS) for obsessive-compulsive disorder (OCD): An exploratory meta-analysis of randomized and sham-controlled trials. J Psychiatr Res. 2013; 47:999-1006.

2. Nota, J. A., Sharkey, K. M., Coles, M. E. Sleep, arousal, and circadian rhythms in adults with obsessive-compulsive disorder: A meta-analysis. Neurosci Biobehav Rev. 2015; 51:100-107.

\section{A50}

Glutamatergic deficit and negative symptoms: new evidence from the ketamine model of schizophrenia

Stephanie Thiebes ${ }^{1}$, Gregor Leicht ${ }^{1}$ Stiepan Curic', Saskia Steinmann ${ }^{1}$, Nenad Polomac', Iris Eichler', Lars Eichler², Christian Zöllner ${ }^{2}$, Jürgen Gallinat ${ }^{3}$, Ileana Hanganu-Opatz ${ }^{4}$, Christoph Mulert ${ }^{1}$

'Psychiatry Neuroimaging Branch, Department of Psychiatry and Psychotherapy, University Medical Center Hamburg-Eppendorf, 20251Hamburg, Germany; ${ }^{2}$ Department of Anesthesiology, University Medical Center Hamburg-Eppendorf, 20251 Hamburg, Germany;

${ }^{3}$ Department of Psychiatry and Psychotherapy, University Medical Center Hamburg-Eppendorf, 20251 Hamburg, Germany; ${ }^{4}$ Developmental Neurophysiology, Institute of Neuroanatomy, University Medical Center Hamburg- Eppendorf, 20251 Hamburg, Germany

Correspondence: Stephanie Thiebes (s.thiebes@uke.de) Neuropsychiatric Electrophysiology 2016, 2(Suppl 1):A50 
Targeting the N-methyl-D-aspartate-receptor (NMDAR) is a major approach for treating negative symptoms of schizophrenia. The ketamine model of schizophrenia has the advantage of comprehensively producing schizophrenia like symptoms such as positive, cognitive and negative symptoms in healthy volunteers. The amplitude of the Mismatch Negativity (MMN), a neurophysiological parameter related to infrequent stimuli, is known to be significantly reduced in schizophrenic patients but also in healthy controls receiving ketamine $[1,2]$. Accordingly, it was the aim of the present study to investigate whether changes of MMN during ketamine administration are related to the emergence of negative symptoms in healthy subjects. Therefore, we examined the impact of ketamine on MMN amplitudes and its sources (sources localization approach: low resolution electromagnetic tomography (LORETA)) by means of 64-channel electroencephalography (EEG) recording during performance of an auditory MMN paradigm and assessed the psychopathological status using the Altered State of Consciousness (5D-ASC) Rating Scale and the Positive and Negative Syndrome Scale (PANSS). Twenty-four male, healthy volunteers were measured with pharmacological EEG using a single-blind, randomized, placebo-controlled crossover design.

We identified significant changes of the MMN response, to both duration and frequency deviants, under ketamine condition as well as a significant increase in all PANSS scores. Reductions of MMN amplitudes were significantly correlated with more pronounced negative symptoms, assessed by the PANSS.

Accordingly, the MMN might represent a biomarker for negative symptoms in schizophrenia related to an insufficient NMDAR system and could be used to identify schizophrenia patients with negative symptoms due to NMDAR dysfunction and thus to determine a maximal benefit of drugs modulating neurotransmission at the NMDAR.

\section{Competing interests}

The authors declare that they have no competing interests.

\section{References}

1. Shelley AM, Ward PB, Catts SV, Michie PT, Andrews S, McConaghy N. Mismatch negativity: an index of a preattentive processing deficit in schizophrenia. Biol Psychiatry. 1991; 30(10):1059-1062.

2. Umbricht D, Koller R, Vollenweider FX, Schmid L. Mismatch negativity predicts psychotic experiences induced by NMDA receptor antagonist in healthy volunteers. Biol Psychiatry. 2002; 51(5):400-406.

\section{A51}

Isolated epileptiform discharges in psychiatry: outcomes in an integrative practice

Ronald J Swatzyna', Jay D Tarnow', Robert P Turner², Alexandra J Roark', Erin K Maclnerney', and Gerald P Kozlowski ${ }^{3}$

${ }^{1}$ Tarnow Center for Self-Management, Houston, TX, USA; ${ }^{2}$ Network Neurology LLC, Charleston, SC, USA; ${ }^{3}$ Saybrook University, Oakland, CA, USA

Correspondence: Ronald J Swatzyna (drron@tarnowcenter.com) Neuropsychiatric Electrophysiology 2016, 2(Suppl 1):A51

\section{Background}

The search for biomarkers that can inform medication decisions in neuropsychiatric disorders is a goal of the Research Domain Criteria project under the National Institute of Mental Health. Isolated epileptiform discharges (IEDs) may be such a biomarker. IEDs have been linked to increased psychopathology that traverses many diagnoses [1]. It has been suggested that IEDs may represent an epiphenomenon with an etiology of unappreciated significance [2]. The literature suggests that anticonvulsants should be considered when IEDs are identified [3, 4]; however, outcome studies have yet to be published. This study investigates the predictive value of IEDs as a biomarker for the use of anticonvulsants on a large cohort of patients. Method

We reviewed refractory cases from a large multidisciplinary practice whose EEG readings contained IEDs and were subsequently medicated with anticonvulsants by the clinic's psychiatrist. The psychiatrist's follow up progress notes were assessed to determine the impact of adding anticonvulsants. Ratings were based on clinical presentation and reported in three categories: Improved, unchanged, and more severe. There were two exclusion criteria: a prior diagnosis of seizure disorder and a history of prior treatment with anticonvulsants. Of the 735 patients in our database, 325 (44.22 \%) were identified with IEDs. The final sample was comprised of 76 refractory cases. The study included 61 males (80.26\%) and 15 females (19.74\%) ages 5 to 52 .

Results

Of the 76 cases treated with anticonvulsants, the vast majority were found to be improved in follow-up progress notes: Improved 65 (85.53 \%), Unchanged 6 (7.89 \%), and More Severe 5 (6.58 \%).

Conclusions

IEDs predict positive treatment outcome to anticonvulsant medication and may not only represent a biomarker for medication selection but also a step towards an evidence-based diagnosis. This review serves as the first large outcome study in which patients with IEDs were treated with anticonvulsants. Our findings suggest that EEG screening should be utilized in all refractory cases regardless of age, gender, or diagnosis. When IEDs are identified, anticonvulsants should be considered as a treatment option.

Consent to publish

This study does not contain details relating to individual participants.

Competing interests

The authors declare that they have no competing interests.

References

1. Zimmerman EM, Konopka LM. Preliminary findings of single- and multifocused epileptiform discharges in nonepileptic psychiatric patients. Clin EEG Neurosci. 2014;45(4):285-292. doi: 10.1177/1550059413506001.

2. Boutros NN. Standard EEG: A research roadmap for neuropsychiatry. Cham, Switzerland: Springer; 2013:135-147chap14.

3. Millichap JG, Millichap JJ, Stack CV. Utility of the electroencephalogram in attention deficit hyperactivity disorder. Clin EEG Neurosci. 2011;42(3):180-184. doi: 10.1177/155005941104200307.

4. Swatzyna RJ, Tarnow JD, Roark A, Mardick J. The utility of EEG in attention deficit hyperactivity disorder: a replication study. Clin EEG Neurosci. 2016. doi: 10.1177/1550059416640441.

A52

Do cannabinoid antagonists affect cognition? SLV326 induces changes in theta and gamma bands in active rats Martin F J Perescis ${ }^{1,2}$, Natasja de Bruin ${ }^{3}$, Liesbeth Heijink ${ }^{4}$, Chris Kruse ${ }^{5}$, Lyudmila Vinogradova ${ }^{6}$, Annika Lüttjohann ${ }^{7}$, Gilles van Luijtelaar ${ }^{1}$, Clementina M van Rijn ${ }^{1}$

${ }^{1}$ Radboud University, Nijmegen, The Netherlands; ${ }^{2}$ HAS University of Applied Sciences, 's Hertogenbosch, The Netherlands; ${ }^{3}$ Fraunhofer Institute for Molecular Biology and Applied Ecology IME, Project Group Translational Medicine \& Pharmacology TMP, Frankfurt am Main, Germany; ${ }^{4}$ Astellas Pharma Europe R\&D, Leiden, The Netherlands; ${ }^{5}$ University of Amsterdam, Amsterdam, The Netherlands; ${ }^{6}$ Institute of Higher Nervous Activity and Neurophysiology, Russian Academy of Sciences, Moscow, Russia; ${ }^{7}$ Westfälische Wilhelms Universität Münster, Münster, Germany

Correspondence: Martin F J Perescis (m.perescis@donders.ru.nl) Neuropsychiatric Electrophysiology 2016, 2(Suppl 1):A52

Cannabinoid CB1 antagonists have been investigated for possible treatment of e.g. obesity-related disorders. However, clinical application was halted due to their symptoms of anxiety and depression. In addition to these adverse effects, we have shown earlier that chronic treatment with the CB1 antagonist rimonabant may induce convulsive seizures which were EEG-confirmed. However, due to the wide distribution of $C B 1$ receptors throughout the CNS, it is highly unlikely that chronic blocking of the CB1 receptor is only manifested in seizures. $\mathrm{CB} 1$ agonists have been described to alter the EEG frequency spectrum. No such data are available for CB antagonists.

In a regulatory repeat-dose toxicity study "muscle spasms" were observed in Wistar rats, daily dosed with the CB1 receptor antagonist SLV326 during 5 months. In selected SLV326-treated and control 
animals, EEG and behavior were monitored for $24 \mathrm{~h}$. Subsequently, random segments of the interictal EEG were selected, totaling 20 min per animal. These segments were assigned to subsets of 'active state' or 'passive state', based on Passive Infrared (PIR) motion detection. Spectral information was calculated using a Fast Fourier Transformation analysis.

$25 \%$ of SLV326 treated animals showed, EEG-confirmed, spontaneously occurring generalized convulsive seizures, whereas all controls were seizure-free. The behavioral signs of the seizures were typical for a limbic origin.

The frequency spectrum of the interictal EEG of the treated rats showed a lower theta peak frequency, as well as lower gamma power compared to the controls. These frequency changes were state-dependent: they were only found during high locomotor activity. However, the treatment did not affect the amount of locomotor activity itself.

Apart from confirming our previous finding that long-term blockade of the endogenous cannabinoid system can provoke limbic seizures in otherwise healthy rats, this study shows that SLV326 alters the frequency spectrum of the EEG, but only when rats are highly active. It is therefore likely that the EEG effects caused by SLV326 are linked to higher order behavior that might be present during locomotion. Theta rhythm is shown to be a marker of complex behavior, and gamma rhythm is typically associated with cognitive functions. Therefore, these observations suggest that $C B$ antagonists might have effects on complex behavior and cognition.

A53

EEG connectivity on sources in male non-smokers after nicotine administration during resting-state

Paolo Ranzi ${ }^{1}$, Jan A Freund ${ }^{2,4}$, Christiane M Thiel ${ }^{3,4}$ \& Christoph S Herrmann ${ }^{1,4}$

${ }^{1}$ Experimental Psychology Group, Department of Psychology, Cluster of

Excellence "Hearing4all", European Medical School, Carl von Ossietzky

University, Oldenburg, Germany; ${ }^{2}$ Institute for Chemistry and Biology of the

Marine Environment, Carl von Ossietzky University, Oldenburg, Germany;

${ }^{3}$ Biological Psychology Group, Department of Psychology, Cluster of

Excellence "Hearing4all", European Medical School, Carl von Ossietzky

University, Oldenburg, Germany; ${ }^{4}$ Research Center Neurosensory Science, Carl von Ossietzky University, Oldenburg, Germany

Correspondence: Paolo Ranzi (paolo.ranzi@uni-oldenburg.de)

Neuropsychiatric Electrophysiology 2016, 2(Suppl 1):A53

\section{Background}

New developments in pharmacology are based on non-invasive neuroimaging, particularly by leveraging and optimizing techniques and methodologies already validated in basic neuroscience. Particularly interesting is the use of connectivity measures of electromagnetic oscillatory activity regarding the modulation of vigilance. Indeed, several recent anesthesiology papers found a connection between vigilance and the connectivity measured from electromagnetic oscillatory activity [1]. We present an EEG connectivity study aimed to establish whether nicotine-induced modulations of vigilance impact connectivity.

\section{Methods}

EEG activity was recorded in an eyes-open and eyes-closed condition before and after drug administration in thirty healthy male nonsmokers. The subjects were randomly allocated either to a nicotine group (14 subjects, $7 \mathrm{mg}$ transdermal nicotine) or to a placebo group. A double-blind placebo-controlled design was implemented. With source reconstruction procedure (eLORETA algorithm), we extracted thirteen time-series representing thirteen regions of interest (ROIs). Each ROI was anatomically precise and belonged to the resting-state network which seems to be modulated specifically by eyes-open and eyes-closed activity. In the literature such restingstate network is labeled as the Default Mode Network. Here we conducted connectivity analysis (renormalized Partial Directed Coherence, rPDC) on the ROls' time-series, focusing on the frequency range of 8.5 to $18.4 \mathrm{~Hz}$. Such frequency range was further subdivided into three frequency bands $\left(a_{1}, a_{2}\right.$ and $\left.\beta_{1}\right)$ in order to comply with current EEG standards of practice.
Results

Our connectivity analysis found that during eyes-closed, nicotine decreased feed-back connectivity (from precentral gyrus to precuneus, angular gyrus, cuneus and superior occipital gyrus) at $10.5-12.4 \mathrm{~Hz}\left(\mathrm{a}_{2}\right)$. During eyes-open, no significant results were found at any frequency range. Conclusions

We interpreted the results by help of previous anesthesiology literature about an anti-correlated relationship between feed-back and feedforward connectivity. Such relationship emerged by pharmacologicallyinduced sedation during eyes-closed condition. Our results suggest that nicotine potentially increases the level of vigilance. Such nicotine-effect is particularly prominent during the eyes-closed condition.

\section{Competing interests}

Nothing to declare.

\section{References}

1. Maksimow A, Silfverhuth M, Langsjo J, Kaskinoro K, Georgiadis S, Jaaskelainen $\mathrm{S}$, et al. Directional connectivity between frontal and posterior brain regions is altered with increasing concentrations of propofol. PloS one. 2014;9(11):e113616.

A54

Withdrawn

\section{Poster presentations}

A55

Modulation of the NMDA receptor function enhances hippocampal network oscillations, connectivity and synaptic LTP in-vivo: A case study with a Glycine Transporter-1 Inhibitor

Ahnaou A, Biermans R, Huysmans H, Drinkenburg WH

Department of Neuroscience Discovery, Janssen Research \&

Development, a Division of Janssen Pharmaceutica NV. Turnhoutseweg

30, B-2340 Beerse, Belgium

Correspondence: Ahnaou A (aahnaou@its.jnj.com)

Neuropsychiatric Electrophysiology 2016, 2(Suppl 1):A55

Hypofunction of N-methyl-d-aspartate receptors (NMDARs) has been associated with deficits in synaptic plasticity and cognitive decline as found in neuropsychiatric and neurodegenerative disorders such as Alzheimer's disease. Glycine and D-serine are endogenous ligands of the NMDAR and therapeutic approaches that enhance NMDAR activity through increases in glycine and/or D-serine levels are expected to enhance synaptic strength and to potentially improve have impact on cognition processes. The present in-vivo study investigated whether positive modulation of brain glycine levels, through modulation by the glycine transporter 1 (GlyT1) inhibitor SSR504734, affects network connectivity and long-term potentiation (LTP) at the hippocampus. For in-vivo network oscillations and connectivity, multichannel EEG recordings were performed in conscious Sprague-Dawley rats from frontal cortical, hippocampal CA1 and CA3 and dentate gyrus (DG) structures after subcutaneous administration of vehicle or SSR504734 (2.5, 10 and $40 \mathrm{mg} / \mathrm{kg})$. For hippocampal synaptic plasticity, rats were anesthetized with urethane and recording and stimulating electrodes were inserted at the DG and at the medial perforant pathway (MPP), respectively. Population spike (PS) amplitudes (PSA) and excitatory postsynaptic potential (EPSP) slope were measured before and 2-h after high-frequency stimulation (HFS).

SSR504734 (at $40 \mathrm{mg} / \mathrm{kg}$ ) elicited robust EEG slow theta oscillations $(4-6.5 \mathrm{~Hz})$ at the DG, CA1 and CA3 and in addition slow gamma oscillations $(30-50 \mathrm{~Hz})$ in the frontal areas, next to network coherence changes between frontal and CA1 recording sites, which were dissociated from motor behavior. SSR504734 (at $40 \mathrm{mg} / \mathrm{kg}$ ) enhanced LTP of the PS amplitude after HFS of the MPP synapse, whereas the potentiation of EPSP slope was short-lived.

The present data support the hypothesis on a facilitating role of the NMDARs glycine binding site on network oscillations and synaptic efficacy at the medial perforant path of the DG. Future studies will evaluate novel approaches targeting D-Serine modulatory sites, for 
example by inhibition of the enzyme d-amino acid oxidase (DAAO), which slows the break-down of D-serine, or by its transporter, the alanine-serine-cysteine-1 (Asc-1), the abnormal glio-transmission of which has been linked to synaptic failure in Alzheimer's disease.

\section{A56}

Withdrawn

\section{A57}

Neurofeedback training as a treatment for dyslexia

Parissa Azadi, Stig Hollup

Department of Psychology, Norwegian University of Science and

Technology, Trondheim, Norway

Correspondence: Stig Hollup (stigh@svt.ntnu.no)

Neuropsychiatric Electrophysiology 2016, 2(Suppl 1):A57

Dyslexia is amongst the commonest of neurobiological disorders, affecting about $20 \%$ of children in Norway. Its heterogeneity makes it difficult to establish a single treatment which is suitable for most of the affected. According to the phonological theory of dyslexia, the disorder is caused by a deficit in the representation, storage and recall of speech sounds. Different brain areas have been linked to the phonological deficit by means of different brain imaging techniques, among other quantitative electroencephalography (qEEG).

The aim of this study was to improve reading ability in children with dyslexia by means of individualized neurofeedback training. The study was conducted as a pre-post intervention single-subject design with 5 participants, aged from 13 to 14 years. The intervention consisted of 25 sessions of neurofeedback training, 15 beta/theta frontocentral sessions and 10 individualized sessions, mostly towards the language areas. The effect of the intervention was measured by means of qEEG and the LOGOS (a Norwegian dyslexia assessment battery).

The results showed improvement in reading abilities and phonological skills amongst all participants. Furthermore, qEEG analysis showed increased alpha activity in several brain areas, and normalization of theta and beta activity in comparison to a normative database. An increase of alpha activity may possibly indicate changes in alpha coherence which can be an indication of improved attentional processes. This may explain the improvements in reading and phonological skills. The analysis also confirms the heterogeneity of dyslexia, and the complicity of several brain areas that are involved in dyslexia.

This study is limited by the small number of participants, and the restriction in time (the number of training sessions offered). However, the improvement in reading and phonological skills in this study suggests that neurofeedback training may be an effective and relevant intervention for adolescents with dyslexia. But, further research in this area with larger samples and a larger number of training sessions is required. The study was approved by the regional ethics committee.

\section{Competing interests}

There are no competing interests.

\section{A58}

Mismatch negativity (MMN) in serotonergic model of psychosis induced by psilocybin

Anna Bravermanova ${ }^{1,2}$, Michaela Viktorinova ${ }^{1,3}$, Filip Tyls 1,3, Jakub Korcak ${ }^{1,3}$ Vlastimil Koudelka', Martin Brunovsky ${ }^{1,3}$, Martin Bares ${ }^{1,3}$ and Tomas Palenicek ${ }^{1,3}$ ${ }^{1}$ National Institute of Mental Health, Klecany, Czech Republic; ${ }^{2} 1^{\text {st }}$ Faculty of Medicine, Charles University in Prague, Prague, Czech Republic; ${ }^{3} 3^{\text {rd }}$ Faculty of Medicine, Charles University in Prague, Prague, Czech Republic Correspondence: Anna Bravermanova (anna.bravermanova@nudz.cz) Neuropsychiatric Electrophysiology 2016, 2(Suppl 1):A58

\section{Background}

The auditory MMN (mismatch negativity) is considered to be an index of automatic context-dependent information processing and auditory sensory memory. MMN deficit is a characteristic endophenotype of schizophrenia. A $5-\mathrm{HT}_{2 \mathrm{~A}}$ agonist psilocybin induces acute transient psychotic symptoms and is extensively used as a putative pharmacological model of schizophrenia. Our aim was to investigate the effect of psilocybin on this pre-attentive cognitive functions.

Methods

A double-blind, placebo controlled study design was used. 20 healthy adult volunteers were administered a dose of psilocybin $(0,26 \mathrm{mg} / \mathrm{kg})$ and placebo per os in 2 separate sessions. Auditory MMN was recorded in sound and electrically shielded room, $120 \mathrm{~min}$ after ingestion of psilocybin/placebo. Participants were lying down with their eyes closed in a comfortable setting with two sitters who were present during whole experiment.

MMN

A single deviant paradigm with 1350 standard $(1000 \mathrm{~Hz}, 75 \mathrm{~dB} \mathrm{SPL}$, 100 ms duration) and 75 deviant in frequency $(1200 \mathrm{~Hz}, 75 \mathrm{~dB} \mathrm{SPL}$, $100 \mathrm{~ms}$ ) tones were presented binaurally in regular order when every $20^{\text {th }}$ was deviant tone. Data was acquired with a standard 32-channel digital EEG amplifier BrainScope (unimedis, Prague) with $21 \mathrm{Ag} / \mathrm{AgCl}$ scalp electrodes placed according to the $10 / 20$ system and sampled at $1000 \mathrm{~Hz}$.

Results

Mismatch negativity was calculated by subtracting the average of frequently occurring stimuli from the average of deviants. There were no significant differences in latency, absolute amplitude and area under curve of MMN during psilocybin intoxication compared to placebo. Furthermore, there were no correlations between subjective effects induced by psilocybin (HRS and ASCS) and MMN.

Conclusion

Our results correspond with previous findings [1]. Psilocybin does not affect processing at the level of pre-attentive cognition and the auditory sensory memory.

This effect is probably due to different underlying receptor mechanism as the generation of MMN is strongly dependent on NMDAR dysfunction. Another reason for negative results could be inappropriate timing of recording or insufficient single-deviant paradigm.

This work is supported by Ministry of Health of the Czech Republic, grant nr. 15-29900A. All rights reserved.

\section{References}

1. Umbricht D, Vollenweider F X, Schmid L, Grübel C, Skrabo A, Huber R et Koller R. Effects of 5-HT2A agonist Psilocybin on Mismatch Negativity generation and AX-Continuous Performance Task: Implications for the Neuropharmacology of Cognitive Deficits in Schizophrenia. Neuropsychopharmacology 2003;Jan;28(1):170-81

\section{A59}

Comparison between SMR and Upper Alpha Neurofeedback trainings as a non-pharmacological treatment of ADHD and sleep disorders in children and adolescents

Eléonore Czarik', Hervé Caci ${ }^{2}$, Jean-Paul Laurent ${ }^{3}$

${ }^{1}$ Université Paris 8, Ecole doctorale cognition, langage, interaction, Saint-Denis, France; ${ }^{2}$ Hopitaux pediatriques de Nice Chulenval, Nice, France; ${ }^{3} L u t i n$ cité des sciences et de l'industrie, laboratoire, cognitions humaines et artificielles, chart ea 404, Université Paris 8, Saint-Denis, France

Correspondence: Eléonore Czarik (eleonoreczarik@ymail.com) Neuropsychiatric Electrophysiology 2016, 2(Suppl 1):A59

\section{Background}

About $5 \%$ of school-aged children may have an Attention DeficitHyperactivity Disorder (ADHD), a neurodevelopmental disorder often associated with other comorbid conditions including sleep disorders. ADHD became a public health concern. Psychostimulants are the first line pharmacological treatments for ADHD. However, parents are often reluctant to medicate their children and, additionally, a proportion of patients stop their treatment because of side effects. Nonpharmacological treatments are also available. Recently, improvements of cognitive functioning and hyperactivity level of patients with ADHD have been reported after Neurofeedback trainings with a relative Upper Alpha Power enhancement paradigm. Sensorimotor rhythm (SMR) Neurofeedback has been also proposed to improve 
ADHD symptoms. The aim of this study is to compare the benefits of Upper Alpha and SMR trainings on ADHD symptoms and concomitant improvement of sleep.

\section{Methods}

In this controlled and randomized study, 60 French medication-free children and adolescents with ADHD aged from 8 to 15 years old will participate in 30 neurofeedback sessions. They will be assigned to either in either the SMR or the Upper Alpha training group. EEG, ADHD rating scales, cognitive assessment, and actigraphic records will be performed at pre-, mid- and post-training times, and 6 months after the end of protocol. Results

The main expected outcome is the clinical reduction of at least $30 \%$ of ADHD symptoms, and we anticipated the superiority of Upper Alpha training over SMR in reducing hyperactivity levels. Improvement of sleep quality is a secondary outcome.

\section{Conclusion}

To date, no comparison between SMR and Upper Alpha Neurofeedback trainings with a significant number of sessions and enough patients in each group has been conducted. We hope to gain valuable insights into specific effects of both trainings on ADHD symptoms and sleep without any medication. This study would foster the development of research on Neurofeedback and its clinical applications, which are under-investigated in France.

Trial Registration

NID RCB 2016-A00655-46

Keywords

ADHD, Neurofeedback, SMR-Upper Alpha Training, EEG, non pharmacological treatment, sleep disorders

\section{A60}

Effect of Tai-Chi and Cyclic Meditation on hemodynamic responses of the prefrontal cortex

S Deepeshwar', NK Manjunath², M Avinash ${ }^{3}$

${ }^{1}$ Cognitive Neuroscience Laboratory (CNL), Swami Vivekananda Yoga

Anusandhana Samsthana (S-VYASA), 19 Eknath Bhavan, Gavipuram

Circle, K.G. Nagar, Bangalore India - 560019; ${ }^{2}$ Swami Vivekananda Yoga

Anusandhana Samsthana (S-VYASA), 19 Eknath Bhavan, Gavipuram

Circle, K.G. Nagar, Bangalore India - 560019; ${ }^{3}$ Shanghai Sports University,

Shanghai, China.

Correspondence: S Deepeshwar (deepeshwar.singh@svyasa.org)

Neuropsychiatric Electrophysiology 2016, 2(Suppl 1):A60

\section{Background}

Mind body based meditation techniques called Tai-Chi Chuan (TCC, a moving meditation) [1] and Cyclic Meditation (CM, a stimulation and relaxation meditation) [2] has been proven to reshape the patterns of brain structures and functional connectivity. TCC practices showed improvement in the brain functions associated with cognition, behavior and health [3]. Similarly, CM also reported improvement in midbrain region [4] associated with better information processing speed or improved motor speed [5].

\section{Method and materials}

We evaluated the effect of Tai-Chi Chuan (TCC, a Chines movement based meditation technique) and Cyclic Meditation (CM, an Indian traditional based stimulation and relaxation meditation technique) on the hemodynamic responses of the prefrontal cortex (PFC) activity and autonomic functions (such as R-R interval (RR-I of heart rate variability and respiration). These two meditation practices were compared with simple walking. Employing 64 channel near infrared spectroscopy (NIRS), we measured hemoglobin concentration change (i.e., Oxyhemoglobin [DHbO], Deoxyhemoglobin [DHbR] and Total hemoglobin change [DTHC]) in the bilateral PFC before and after TCC, CM and Walking in young college students $(n=25$; average mean age $\pm S D ; 23.4 \pm 3.1$ years).

Results

We observed the left PFC activity predominantly modulates sympathetic activity effects during the Tai-Chi whereas CM showed changes on right PFC with vagal dominance. However, the changes in oxyhemoglobin and total blood volume change after Tai-Chi was significant higher $(p<0.05$, spm t-maps) on left hemisphere, whereas after $\mathrm{CM}$, there were a significant increase in oxyhemoglobin $(p<0.01)$ with a decrease in deoxyhemoglobin $(p<0.05)$ on right PFC. The normal walking showed decrease in Oxyhemoglobin with increase in deoxyhemoglobin on left PFC. The autonomic functions result showed a significant increase in RR- interval $(p<0.05)$ along with significant reduction in $H R(p<0.05)$ in $C M$ whereas Tai-chi session showed significant increase in HR $(p<0.05)$ when compared to walking session. Within group analysis showed a significant reduction in RR-I and significant increase in HR both in Tai-chi and walking sessions. The CM showed there were a significant improvement in RR-interval of HRV $(p<0.01)$ with reduction of heart rate and breath rate $(p<0.05)$.

\section{Conclusions}

The result suggested that Tai-Chi and CM both have positive effect on left and right prefrontal cortex and increase sympathovagal balance (alertful rest) in autonomic nervous system activity.

Keywords

Tai-Chi-Chuan (TCC); Yoga; Cyclic Meditation (CM); Walking; Prefrontal Cortex (PFC); Heart Rate Variability (HRV)

\section{References}

1. J. L. W. Robins, R. K. Elswick, and N. L. McCain, "The story of the evolution of a unique tai chi form: origins, philosophy, and research.," J. Holist. Nurs., vol. 30, no. 3, pp. 134-46, 2012.

2. P. Subramanya and S. Telles, "A review of the scientific studies on cyclic meditation.," Int. J. Yoga, vol. 2, no. 2, pp. 46-48, Jul. 2009.

3. G.-X. Wei, H.-M. Dong, Z. Yang, J. Luo, and X.-N. Zuo, "Tai Chi Chuan optimizes the functional organization of the intrinsic human brain architecture in older adults.," Front. Aging Neurosci., vol. 6, p. 74, Jan. 2014.

4. P. Subramanya and S. Telles, "Changes in midlatency auditory evoked potentials following two yoga-based relaxation techniques.," Clin. EEG Neurosci., vol. 40, no. 3, pp. 190-5, Jul. 2009.

5. P. Subramanya and S. Telles, "Performance on psychomotor tasks following two yoga-based relaxation techniques.," Percept. Mot. Skills, vol. 109, no. 2, pp. 563-76, Oct. 2009.

A61

Withdrawn

A62

EnkephaloVision: fast dynamic EEG analysis in combination with eye tracking for efficacy testing of plant-derived and low triturated homeopathic drugs.

Wilfried Dimpfel (dimpfel1945@web.de)

Justus-Liebig-University Giessen, Giessen, Hessen, 35390 Germany

Neuropsychiatric Electrophysiology 2016, 2(Suppl 1):A62

Efficacy testing of plant-derived and homeopathic drugs still remains a challenge in pharmacology. In the past several neurophysiological techniques have been successfully applied. However, interpretation of spectral power values with respect to different brain regions is contradictory. Conventional quantitative EEG analysis uses averaged data from epochs of 2 or $4 \mathrm{~s}$. Analysis of shorter epoch length of $364 \mathrm{~ms}$ has been achieved by definition of specific frequency ranges [1]. Surprisingly, focal spectral power within these short periods reached tremendous values (up to $9000 \%)$ at single brain regions when compared to the average of other assessed regions (global median) [2]. In order to learn more about these short periods of high electric activity, EEG analysis was combined with eye tracking. The eye tracking software served to present different cognitive and emotional audio-visual challenges in series. Synchronization of the gaze overlay video from the eye tracking with screen capture of the online quantitative EEG analysis was achieved by starting the recording with a gong. The combined technology has been published [3]. Synchronized scenes were evaluated before and after intake of the preparations. In the presence of cognition activating drugs (i.e. Zembrin $^{\oplus}$ ) more flashing of delta $(1.375-4.125 \mathrm{~Hz})$ and theta spectral power $(4.125-6.875 \mathrm{~Hz}$ ) was observed in frontal brain in comparison to placebo during performance of psychometric testing. In the presence of calming drugs (i.e. plant-derived drug Pascoflair ${ }^{\circledR}$ ) more flashing of alpha1 spectral power $(6.875-9.625 \mathrm{~Hz})$ was recognized in comparison 
to placebo. The same increase of spectral alpha1 power was detected after intake of 6 homeopathic Calmvalera Hevert tablets at a time. Since about 3 pictures per second are difficult to follow, slow motion videos will be presented. Finally, averaged data were fed into a discriminant analysis. Comparison of several plant-derived and homeopathic drugs with each other revealed for example projection of data from 3 calming drugs in close vicinity to each other.

\section{References}

1. Dimpfel, W., Hofmann, H-C. (2014) Neurocode-Tracking Based on Quantitative Fast Dynamic EEG Recording in Combination with Eye-Tracking. World Journal of Neuroscience 4, 106-119.

2. Dimpfel, W., Chiegoua Dipah, G.N., Gericke, N. (2016) EnkephaloVision: Anatomical Functionality Indicated by Ultrashort Transient Regional EEG Spectral Power Changes during Cognitive and Emotional Challenges. World Journal of Neuroscience, 6, 90-108.

3. Dimpfel, W. (2015) Drug Discovery and Translational Medicine Based on Neurophysiological Techniques. A holistic approach to saving animals. Verlag Books on Demand, Norderstedt, Germany.

A63

Quantitative EEG assessment of students with ADHD undergoing neurofeedback training

Caroline Dupont ${ }^{1,2}$, Brendan Parsons ${ }^{1}$, Hélène Brisebois ${ }^{2}$, Andrea Szabo ${ }^{2}$ ${ }^{1}$ Département de psychologie, Université de Montréal, Montréal, Canada; ${ }^{2}$ Centre Alpha Neuro, Collège Montmorency, Laval, Canada

Correspondence: Caroline Dupont (caroline.dupont2@gmail.com)

Neuropsychiatric Electrophysiology 2016, 2(Suppl 1):A63

Several studies have demonstrated abnormalities in quantitative electroencephalogram (qEEG) in children and adults with ADHD. Based on these findings, neurofeedback training (NFT) has emerged has a new treatment option for ADHD. In this preliminary study, qEEG was used to assess the efficacy of NFT training for college students with ADHD. Participants received computer attention training intervention using NFT two times a week over a period of four months. A group of college students with ADHD who did not undergo NFT training was used as a control group. Brain activity was measured using qEEG prior to, midway through, and post NFT training. ADHD behavioral symptoms were also assessed pre- and post- training using the Conners' Adult ADHD Rating Scale (CAARS-S:L) and the IVA-2. Changes in qEEG were detected following NFT. Significant changes in restingstate brain activity were observed in the experimental group. Participants who underwent training demonstrated significant decreases in absolute power across a wide spectrum of frequency bands (delta, theta, alpha and beta) as well as a relative decrease in alpha activity and increase in delta and beta activity. In order to identify if anomalous patterns of brain activity were related to symptoms of ADHD, neuroelectric measures were compared to behavioral measures. Changes in neural activity in the experimental group correlate with improvements in ADHD symptoms. Although further research is warranted to determine the exact impact of NFT on the neural correlates of ADHD, these preliminary findings suggest that it might be a promising cognitive training treatment for students with ADHD.

Keywords

qEEG, neurofeedback, ADHD, ADD

\section{A64}

Improving drug discovery using brain oscillations as biomarkers for movement disorders

V Duveau, B Pouyatos, R Maury, B Mandé-Nidergang, C Bouyssières, C Roucard, Y Roche

SynapCell SAS, Bâtiment Biopolis, 5 avenue du Grand Sablon, 38700 La Tronche, France

Correspondence: $\vee$ Duveau (vduveau@synapcell.fr)

Neuropsychiatric Electrophysiology 2016, 2(Suppl 1):A64

Movement disorders represent a group of neurological syndromes characterised by an alteration of voluntary and/or automatic movements. Here, we focused on Parkinson's disease (PD) and essential tremor (ET), which are the most common forms of movement disorders.

Motor symptoms of Parkinson's disease result from a dysfunction of cortico-basal ganglia circuits mainly due to dopaminergic neurons death in the substantia nigra pars compacta. A hypersynchronization of beta frequency oscillatory activity in these circuits has been described in both patients and animal models of the disease.

Essential tremor (ET) is characterized by the symptom of action tremor (which intensifies when the affected muscles are used). ET typically involves a tremor of the arms, hands or fingers. The classically-used animal model of ET is generated by the administration of the beta-carbolin harmaline in mice. Harmaline induces action tremors lasting several hours and the classical read-out is the recording of behavioural tremor frequency that occurs between 8 to $10 \mathrm{~Hz}$. The aim of this poster was to provide two examples of the use of brain oscillations in preclinical drug development for movement disorders. Here, 1) we assessed the use of aberrant cortical oscillations in the unilateral 6-OHDA injected rat as translational biomarkers for drug development in PD, and 2) studied the effect of harmalineinduced tremor on cortical and cerebellar oscillatory activities. The sensitivity of these functional biomarkers was challenged with the reference drugs for each pathology.

In the 6-OHDA rat model of PD, we found a prominent beta band $(\sim 30 \mathrm{~Hz})$ in the motor cortex, which was inexistent in control SpragueDawley rats. Acute injection of the dopaminergic receptor agonist LDOPA ( 6 and $20 \mathrm{mg} / \mathrm{kg}$ ) induced body rotations along with a significant reduction of the beta band. This treatment also induced a prominent $80-100 \mathrm{~Hz}$ gamma increase. By contrast, the D2/D3/D4 agonist ropinirole at $0.2,0.4$, and $0.8 \mathrm{mg} / \mathrm{kg}$ also decreased the beta band but caused only a slight gamma band increase.

We found that administration of harmaline $(10-20-30 \mathrm{mg} / \mathrm{kg})$ in male C57BL/6 J mice dose-dependently increased the cortical and cerebellar power in a wide $15-60 \mathrm{~Hz}$ frequency range, along with action tremors. Pre-treatment with $20 \mathrm{mg} / \mathrm{kg}$ propranolol, one of the firstline medications used in ET patients attenuated the tremors and decreased the $35-60 \mathrm{~Hz}$ range.

In this study, we identified aberrant EEG oscillations in two rodent models of movement disorders. These oscillations and their pharmacological modulation may represent predictive biomarkers for the identification, selection and validation of new therapeutics in movement disorders.

A65

Telemetric electroencephalography (EEG) and in vivo microdialysis to study dopaminergic hyperactivity in freely moving rats

Boris Ferger, Patrizia Voehringer

CNS Diseases Research, Boehringer Ingelheim Pharma GmbH \& Co. KG, Biberach/Riß, Germany

Neuropsychiatric Electrophysiology 2016, 2(Suppl 1):A65

Dopamine is a key regulator of cognition, mood, reward and movement in the human and rodent brain. The dopamine homeostasis is tightly controlled by the dopamine transporter (DAT), which is a target for addictive drugs (such as cocaine and amphetamine), and therapeutic antidepressants.

The present study was designed to investigate the effects of cocaine in freely moving rats using telemetric electroencephalography (EEG) to monitor a hyperdopaminergic state. Additionally, in vivo microdialysis in the nucleus accumbens shell was carried out to measure extracellular cocaine levels and dopamine itself by LC-MS-MS and HPLC coupled to electrochemical detection. Behaviour was assessed by an automated motor activity system using light beam interruptions.

Cocaine $(5,10$ and $15 \mathrm{mg} / \mathrm{kg}$, i.p.) dose dependently induced an increase in motor activity, which reached its maximum level after $20 \mathrm{~min}$ and lasted for $90 \mathrm{~min}$. In addition, cocaine appeared to affect the EEG power spectrum, increasing gamma frequency band power up to 60 min after administration, whilst causing a decrease of power in delta, theta, alpha, and beta frequencies. Maximum cocaine levels measured from the dialysates appeared $30 \mathrm{~min}$ after dosing 
(300 nmol/l) and extracellular dopamine levels showed a peak concentration at $30 \mathrm{~min}$ and then returned to basal levels 120 min later.

In conclusion, these results indicate that cocaine induces an increase in dopaminergic transmission in the nucleus accumbens shell, and as expected, produces hyperactivity. The effect observed on the EEG frequency bands and in vivo microdialysis could serve as a physiological biomarker of target engagement studies and to set up a PK-PD relationship in drug discovery research.

\section{A66}

Effects of clozapine on auditory steady-state response in schizophrenia

Inga Griskova-Bulanova', Sigita Melynyte ${ }^{1}$, Kastytis Dapsys ${ }^{2}$, Aleksandras Voicikas'

'Department of Neurobiology and Biophysics, Vilnius University, Vilnius, Lithuania; ${ }^{2}$ Republican Vilnius Psychiatric Hospital, Vilnius, Lithuania Correspondence: Inga Griskova-Bulanova (i.griskova@gmail.com) Neuropsychiatric Electrophysiology 2016, 2(Suppl 1):A66

Auditory steady-state responses (ASSR) provide a non-invasive technique to assess neural synchrony at a particular frequency. Attenuated phase-locking (PLI) of ASSRs in gamma frequency range is observed in schizophrenia and in animal models for psychosis [1]. State-sensitivity of $40 \mathrm{~Hz}$ ASSRs has been shown for schizophrenia, where PLIs increased with eyes closure in patients [2]. The effect of clozapine, which is prescribed in cases of treatment-resistant schizophrenia, on ASSR in humans is not clear. The aim of this study was to evaluate the effect of clozapine use on phase-locking of $40 \mathrm{~Hz}$ ASSR and state-sensitivity in schizophrenia patients.

48 male patients with schizophrenia (according to ICD-10 criteria) were recruited from the in-patients of Republican Vilnius Psychiatric Hospital. Patients were divided into two groups: (1) resistant to standard antipsychotic medication and treated with clozapine (Cloz, $\mathrm{n}=23$ ); and (2) responsive to standard antipsychotic treatment $(\mathrm{NCloz}, \mathrm{n}=25)$. ASSRs to click stimuli at $40 \mathrm{~Hz}$ were recorded using 9 channels in eyes open and eyes closed conditions, with 60 stimuli presented binaurally per condition. After conventional cleaning procedures, epochs of $700 \mathrm{~ms}$ were created starting at $100 \mathrm{~ms}$ prior to the stimulus onset and lasting for $600 \mathrm{~ms}$ post-stimulus. ASSRs were analyzed from Cz location, showing maximal activity. Mean phase-locking index (PLI) within $38-42 \mathrm{~Hz}$ window was calculated for $100 \mathrm{~ms}$ bins and subjected to RM-ANOVA with time bin and task as within-subjects factors and group as a between-subjects factor.

Significant interaction of condition (eyes open vs eyes closed) and group (Cloz vs NCloz) factors $(p=0.038)$ was observed. This suggests that in Cloz group subjects tended to have lower PLIs in open eyes $(p=0.08)$, which increased with eyes closure $(p<0.001)$. In NCloz group, PLls did not change with eyes closure $(p>0.05)$.

Our data propose that state-sensitivity of $40 \mathrm{~Hz}$ ASSRs vary depending on the treatment in patients with schizophrenia, subject receiving clozapine showing response increase with eyes closure in contrast to those on standard antipsychotic treatment.

\section{Competing interests}

The author declare that they have no competing interests

Research was supported by the grant MIP-009/2014 from Lithuanian Research Council.

\section{References}

1. O'Donnell BF, Vohs JL, Krishnan GP, Rass O, Hetrick WP, Morzorati SL. The auditory steady-state response (ASSR): a translational biomarker for schizophrenia. Suppl Clin Neurophysiol. 2013;62:101-12.

2. Griskova-Bulanova I, Dapsys K, Maciulis V, Arnfred SM. Closed eyes condition increases auditory brain responses in schizophrenia. Psychiatry Res Neuroimaging. 2013;211(2):183-5.
A67

Neuro-Cardiac-Guided TMS (NCG TMS): a new and cost-effective method for accurately localizing the DLPFC in the treatment of depression

Tabitha Iseger ${ }^{1,2}$ \& Martijn Arns ${ }^{1,2,3}$

${ }^{1}$ Dept. of Experimental Psychology, Utrecht University, Utrecht, The

Netherlands; ${ }^{2}$ Research Institute Brainclinics, Nijmegen, The Netherlands;

${ }^{3}$ neuroCare Group, Munich, Germany

Correspondence: Tabitha Iseger (tabitha@brainclinics.com)

Neuropsychiatric Electrophysiology 2016, 2(Suppl 1):A67

\section{Background}

The efficacy of rTMS in the treatment of major depressive disorder (MDD) has been well established in recent years. Most studies to date have employed the ' $5-\mathrm{cm}$ ' rule for targeting stimulation of the Dorsolateral Prefrontal Cortex (DLPFC). New variations and improvements of this targeting technique include a ' $6-\mathrm{cm}^{\prime}$ ' rule, the Beam-F3 method, and neuronavigated rTMS. Furthermore, it has been proposed that the efficacy of rTMS in MDD is more related to stimulating the area that is functionally connected to the subgenual anterior cingulate cortex (sgACC) rather than to specific cortical areas (Fox et al., 2012). Therefore, we set-out to develop and test a new method that employs knowledge about the functional role of the sgACC to establish in real time if the right cortical area is targeted.

Method

Several studies have shown that areas in the ventromedial prefrontal cortex are involved in parasympathetic regulation such as heart rate and respiration, and that neurostimulation of these areas led to heart rate decreases (Makovac et al., 2016), most likely through connectivity with the nervus vagus. Therefore, based on the notion that rTMS aims to transsynaptically stimulate the sgACC, we used electrocardiogram (ECG) R-peak triggered single pulse TMS to various frontal locations to establish the location that most consistently resulted in a lengthening of the R-R latency (reflective of a heart rate deceleration). This method of Neuro-Cardiac-Guided TMS or NCG TMS thus could be the equivalent of what the Motor Threshold is for the motor system, but then for the DLPFC with heart rate as an output.

Results

First preliminary results using a burst of $10 \mathrm{~Hz}$ TMS stimulation demonstrated that in a subject with a relatively large head circumference, no response was found at the ' $5 \mathrm{~cm}^{\prime}$ site (corresponding to FC4 in this subject), whereas the F4 location did result in a consistent heart rate deceleration. More data are currently being collected using a single pulse R-peak triggered approach and data will be presented. This method is pending for patent. Dutch Patent office: P100241NL00 Conclusions

In the treatment of MDD, Neuro-Cardiac-Guided TMS has the potential to become the equivalent of the 'motor threshold' for the DLPFC, and thereby would be a cost-effective and easy to use method for localizing the right stimulation target in the treatment of MDD, and also serve as a real-time control of adequate coil contact in patients undergoing rTMS treatment.

A68

Electroencephalogram connectivity in frontal networks to predict outcome of electroconvulsive therapy in major depressive disorder Alexandra Kirsten, Sebastian Olbrich

Department of Psychiatry, Psychotherapy and Psychosomatics, University of Zurich, Zurich, Switzerland

Correspondence: Alexandra Kirsten (alexandra.kirsten@puk.zh.ch)

Neuropsychiatric Electrophysiology 2016, 2(Suppl 1):A68

\section{Background}

Major depressive disorder (MDD) is a common and potentially lethal disorder affecting up to $14 \%$ of all persons worldwide. However, $1 / 3$ to $2 / 3$ of patients are non-responders to first line therapy [1]. Even the electroconvulsive therapy (ECT) as the option of choice in therapyresistant MDD still shows a high proportion of non-responders [2]. Due to the invasive nature of the ECT it would be desirable to know which subjects are likely to respond. In case of a predicted non-response to 
$E C T$, e.g. by means of electrophysiological electroencephalogram (EEG) parameters, other therapies of MDD (e.g. augmentation, polypharmacy etc.) could be chosen.

\section{Methods}

In this study, we retrospectively analysed two minute resting state EEG from patients with MDD who underwent ECT (4-12 sessions with 3/week) between 2005-2015 at the University Hospital of Zurich. Following several lines of evidence, we hypothesized altered non-linear connectivity in frontal networks including subgenual-, dorsolateraland medio- prefrontal cortices being predictive for treatment outcome. Symptom severity and response/remission rates were assessed using the Global Clinical Impression (GCl) rating scale. Source estimates and connectivity measures were mapped using Low Resolution Brain Tomography (LORETA).

Results

Responders in comparison to non-responders showed a significant stronger non-linear connectivity in the frontal network within the EEG delta, alpha 1 and beta 1 frequency bands, while connectivity was weaker in theta, alpha 2, beta 2 and gamma frequency bands. Additionally, there were several non-significant correlations (from $r=.15$ .20) between symptom change and source estimates with e.g. a low midline theta-activity being associated with response to ECT.

\section{Conclusions}

Pre-treatment EEG-connectivity in frontal networks seems to have a predictive value for the efficacy of ECT treatment. Prospective trials and larger study groups are needed to further validate these markers and pave the way for possible usage in the clinical context.

\section{Trial registration}

Project ID: 2016-00562, Swissethics

No competing interests

\section{References}

1. Bromet $E$, Andrade LH, et al. Cross-national epidemiology of DSM-IV major depressive episode. BMC Med. 2011 Jul 26;9:90. DOl: 10.1186/1741-7015-9-90

2. Dierckx B, Heijnen WT, et al. Efficacy of electroconvulsive therapy in bipolar versus unipolar major depression: a meta-analysis. Bipolar Disorders. 2012 Mar 14, Issue 2,146-150. DOI: 10.1111/j.1399-5618.2012.00997.x

\section{A69}

Modulation of the serotonin system in an animal model of psilocin-induced psychosis: a network clustering approach Vlastimil Koudelka', Filip Tylšs, ${ }^{1,2}$, Čestmír Vejmola, ${ }^{1,2}$, Lukáš Kaderábek ${ }^{1,2}$, Václava Piorecká 1,3 , Tomáśs Novák, ${ }^{1,2}$, Martin Brunovský1,2 \& Tomáš Páleníček $k^{1,2}$

${ }^{1}$ National Institute of Mental Health Topolová 748, 250 67, Klecany, Czech Republic; ${ }^{2} 3^{\text {rd }}$ Faculty of Medicine, Charles University in Praque, Prague, Czech Republic; ${ }^{3}$ Faculty of Biomedical Engineering, Czech Technical University in Prague, Prague, Czech Republic

Correspondence: Vlastimil Koudelka (Vlastimil.koudelka@nudz.cz) Neuropsychiatric Electrophysiology 2016, 2(Suppl 1):A69

\section{Introduction}

The present contribution deals with a particular study on animal model of psilocin-induced psychosis. QEEG methods addressing brain activity under certain set of conditions are usually based on bottomup strategy preserving the low-level information e.g. coherence between two electrodes within particular frequency band at certain time after administration of a specific drug. Unfortunately, this approach leads to a combinatorial explosion if the effects of more drugs and their combinations are to be analyzed. This phenomenon makes data difficult to interpret. Here, we propose a way towards top-down strategy based on capturing an interpretable substance fingerprints. We show that unique functional brain clusters coherently modulated by a particular substance are embedded in multidimensional space of coherences and can be extracted by appropriate dimensional reduction technique [1].

Methods

The proposed technique takes coherences from 36 electrode pairs calculated in six discrete frequency bands $(1-40 \mathrm{~Hz})$ at four specific time intervals (base line record, 20-30, 50-60, and 80-90 min post administration) and returns coherent topographic clusters. Our approach can be described in five following steps:

Coherence partial differences are calculated to extract functional changes between time intervals.

Coherence differences are processed by t-Distributed Stochastic Neighbor embedding (t-SNE) [2] to reduce data dimensionality on one hand and encode the original data structure on the other hand.

The silhouette clustering criterion is employed to determine a number of clusters in data [3]

The k-means algorithm categorizes data into clusters determined in step 2 and 3.

Obtained electrode pair clusters are visualized in topographic view. Results

Psilocin clearly shows four functional clusters which are precisely symmetric in topographic view and which exhibit a global maximum of clustering criterion. In similar way, 5HT2A antagonist MDL100907 ,and clozapine result in three clusters. This is in contrast with saline solution exhibiting no clustering and no global extremes of clustering criterion. Generally, all antagonists in combination with psilocin lead to less or no clusters.

\section{Conclusions}

The proposed technique is capable to contrast long term dynamics of coherences and find functional brain structures coherently modulated by a particular drug. This allows us to further study the mechanism behind the psilocin induced functional disconnection - the functional changes are specifically organized. All antagonists seem to compensate the psilocin induced organization. Our next step is to apply the technique in human psilocybin model of psychosis and search a new translation bridge between animal and human models. This study was supported by project "National Institute of Mental Health (NIMH-CZ), number ED2.1.00/03.0078 and the grant AZV from MHCR no. 15-29370A.

I declare no conflict of interests.

References

1. Birjandtalab, J., Pouyan, M. B., \& Nourani, M. (2016, February). Nonlinear dimension reduction for EEG-based epileptic seizure detection. In 2016 IEEE-EMBS International Conference on Biomedical and Health Informatics (BHI) (pp. 595-598). IEEE.

2. Maaten, L. V. D., \& Hinton, G. (2008). Visualizing data using t-SNE. Journal of Machine Learning Research, 9(Nov), 2579-2605.

3. Rousseeuw, P. J. (1987). Silhouettes: a graphical aid to the interpretation and validation of cluster analysis. Journal of computational and applied mathematics, 20, 53-65.

A70

Clonidine Augmentation Therapy in Schizophrenia "CATS-Study": a promising new treatment strategy in resistant schizophrenia Caitlyn Kruiper, Iris E Sommer, Bob Oranje

Dept. of Psychiatry, Brain Center Rudolf Magnus, University Medical Center Utrecht, Utrecht, the Netherlands

Correspondence: Caitlyn Kruiper (C.Kruiper-2@umcutrecht.nl) Neuropsychiatric Electrophysiology 2016, 2(Suppl 1):A70

\section{Background}

Deviations in basic information processing, such as sensory gating, are thought to underlie cognitive deficits in schizophrenia. Treatment with the current first- and second-generation antipsychotics show almost no improvement in these early information processes. In parallel, there is little or no success in treating cognitive deficits with these drugs. In a recently conducted pilot-study from our laboratory we found that administering a single dose of clonidine, a noradrenergic $a 2_{\mathrm{A}}$-receptor agonist, restored sensory gating in patients with schizophrenia to a level that no longer differed from an age and gender matched control group. It is expected that improvement of early information processing leads to improvement in cognition. Goal

Improving currently available antipsychotic medication by normalizing early information processing. 


\section{Methods}

Randomized Clinical Trial (RCT), conform to a randomized, balanced placebo-controlled design with two arms: in condition 1, patients $(n=$ 25) will receive 6 weeks of additional clonidine treatment to their current medication, in condition 2, patients $(n=25)$ will receive 6 weeks of additional placebo treatment to their current medication. In addition, 25 age and gender matched healthy subjects will function as controls. Primary outcome is change in symptom severity, expressed as a change in total score on the Positive and Negative Symptom Scale (PANSS) from baseline to end of the 6-week treatment. Secondary outcomes are changes in cognitive functioning (measured through the Brief Assessment of Cognition in Schizophrenia; BACS and Cambridge Neuropsychological Test Automated Battery; CANTAB), change in GAF (global assessment of functioning) scores and the measurement of various psychophysiological parameters of basic information processing, such as P50 suppression, prepulse inhibition of the startle reflex (PPI) and mismatch negativity (MMN).

Results

In line with our pilot-study it is expected that early information processing will improve. We predict that this will lead to an improvement in cognitive functioning after six weeks, which expectantly leads to lower symptom severity and a better quality of life.

Trial Registration

EudraCT Number: 2014-003008-53

\section{A71}

Isolated epileptiform discharges: an electroencephalographic abnormality underlying medication failure in autism spectrum disorder Erin K Maclnerney', Ronald J Swatzyna', Jay D Tarnow' ${ }^{1}$, Robert P Turner ${ }^{2}$, Alexandra J Roark', and Gerald P Kozlowski ${ }^{3}$

${ }^{1}$ Tarnow Center for Self-Management, Houston, TX, USA; ${ }^{2}$ Network Neurology LLC, Charleston, SC, USA; ${ }^{3}$ Saybrook University, Oakland, CA, USA

Correspondence: Erin K Maclnerney (erinmacinerney@utexas.edu) Neuropsychiatric Electrophysiology 2016, 2(Suppl 1):A71

\section{Background}

Autism Spectrum Disorder (ASD) often presents a treatment challenge due to the variety of symptoms that make each case unique. Medication prescribed to manage ASD associated symptoms such as anxiety, depression, attention issues, and behavioral problems often fail to alleviate symptoms and can produce undesirable side effects. The question is, why are the stimulants, selective serotonin reuptake inhibitors, and antipsychotics prescribed to alleviate these issues [1] effective in some patients but fail in others? The answer could be related to the increased prevalence of electroencephalographic abnormalities in psychiatric patients [2]. The presence of isolated epileptiform discharges (IEDs) may account for the treatment failure of these medications, especially antipsychotics, because these drugs lower seizure threshold, thus resulting in increased epileptiform activity. Electroencephalography (EEG) can be used to document the presence of IEDs that would otherwise go undetected. The purpose of the study was to reveal the prevalence of IEDs in the ASD patient population and to demonstrate the usefulness of the EEG for providing data to psychiatrists, neurologists, and developmental pediatricians to improve medication selection and outcomes for patients with ASD.

Method

The data was obtained from an Institution Review Board approved data archive from a multidisciplinary practice that treats a wide variety of refractory and neuroatypical patients. The study is comprised of 140 non-epileptic children, adolescents, and adults diagnosed with ASD, ages 4 to 25 . A board certified electroencephalographer interpreted the EEGs in order to identify abnormalities.

Results

Of the 140 patients with ASD, 36.4 percent were found to have IEDs after an EEG screening. Chi-square analysis found no significant difference between genders among the three age groups. The findings indicate a high prevalence of IEDs among individuals with ASD.

\section{Conclusion}

Our results find that compared to the healthy population, a large number of patients with ASD have IEDs despite never having a seizure. The findings support the use of EEG in children, adolescents, and young adults with ASD, regardless of gender or age. This is particularly true for those who have failed prior medication attempts with stimulants, antidepressants, and/or antipsychotics. Utilizing the EEG for refractory cases in a psychiatric practice allows for more individualized and precise medication selection.

Consent to publish

This study does not contain details relating to individual participants.

\section{Competing interests}

The authors declare that they have no competing interests.

\section{References}

1. Oswald DP, Sonenklar NA. Medication use among children with autism spectrum disorders. J Child Adolesc Psychopharmacol. 2007;17(3):348355. doi: 10.1089/cap.2006.17303.

2. Zimmerman EM, Konopka LM. Preliminary findings of single- and multifocused epileptiform discharges in nonepileptic psychiatric patients. Clin EEG Neurosci. 2014;45(4):285-292. doi: 10.1177/1550059413506001.

A72

Neuromodulation using maintenance TDCS optimized by qEEG leads to full recovery from myalgic encephalopathy/chronic fatigue syndrome: a case report

Shelly M Menolascino, Mitchell Belgin, Genevieve N Izzo, Lillian E Fisher Washington Square Psychiatry \& TMS, New York, NY, 10011, USA

Correspondence: Shelly M Menolascino

(shellymenomd@washsqtms.com)

Neuropsychiatric Electrophysiology 2016, 2(Suppl 1):A72

\section{Background}

A 61-year-old man with progressive myalgic encephalopathy/chronic fatigue syndrome was referred for neuromodulation. His condition, likely virally induced decades ago, was characterized by recurring periods of extreme fatigue, lasting months at a time. Severe fatigue had become unrelenting over the prior two years, impairing many dimensions of his life. Multiple immunological and neurological workups were negative and fibromyalgia had been ruled out. Patient failed many medically advised approaches, including antidepressants, acupuncture and a gluten-free diet.

Methods

Genetic analysis suggested he would respond to dopaminergic agents and to neuromodulation [1]. Trials of both amphetamines and methylphenidate ultimately failed but modafinil $200 \mathrm{mg}$ did provide partial relief. Distance from the office precluded daily treatment with repetitive transcranial magnetic stimulation (rTMS). Transcranial direct current stimulation (tDCS) was chosen as a safe alternative feasible treatment [2], allowing cumulative, ongoing treatment to target ongoing inflammation. We used neurophysiological state markers of qEEG. Patient was trained with tDCS in the office and then treatment was self-applied at home daily with anode on left dorsal-lateral prefrontal cortex (LDLPFC), cathode on right (RDLPFC), $2 \mathrm{~mA} / \mathrm{min}$, $20 \mathrm{~min}, 40 \mathrm{~mA}$ total dose, using $1.5^{\prime \prime}$ diameter electrode pads. After four weeks, maintenance tDCS sessions were increased to twice daily (6 AM and 12 Noon) and modafinil was lowered to $100 \mathrm{mg}$.

Results

Follow-up qEEG testing was done one year after the initial qEEG when patient was in full recovery. Comparison of pre-treatment and post-treatment qEEG findings show minor improvement in excessive hypercoherent frontal alpha, a substantial $50 \%$ drop in excess left temporal alpha, and a normalization at the very low end of the qEEG spectrum (less than $1 \mathrm{~Hz}$ ). The patient noted: "[this treatment] has given me sustained relief from a chronic fatigue condition from which I've suffered throughout my adult life." 


\section{Conclusions}

Maintenance treatment with daily tDCS and modafinil likely exerted synergistic effects on the brain and immune system. The clinical recovery with notable improved sleep, energy, and ability to tolerate exercise are most likely to be reflected in slow wave oscillation changes. This case supports the need to look more closely at glial as well as neuronal impact, perhaps expanding qEEG to include slow wave markers. Clinicians are eager to have qEEG personalized biomarkers to optimize adjunctive tDCS stimulation in chronic psychiatric and neurological conditions, so often neuroinflammatory in nature [3].

Keywords

tDCS, myalgic encephalopathy, chronic fatigue syndrome, neuroinflammation, qEEG biomarker, personalized medicine

Consent to publish

Informed consent was obtained and the subject's rights were protected.

\section{Competing interests}

No competing interests.

\section{References}

1. Oral A, llieva $E$, Küçükdeveci $A$, Varela $E$, Valero $R$, Verteanu $M$, Christodoulou N. Generalised and regional soft tissue pain syndromes.

The role of physical and rehabilitation medicine physicians. The European perspective based on the best evidence. A paper by the UEMS-PRM Section Professional Practice Committee. Eur J Phys Rehabil Med. 2013: 49: 535-549.

2. Bikson M, Grossman P, Thomas C, Zannou A, Jiang, J, Adnan, et al., Safety of transcranial direct current stimulation: Evidence based update 2016. Brain Stim. 2016; 9: 641-661.

3. Yokoi Y, Sumiyoshi T. Application of transcranial direct current stimulation to psychiatric disorders: trends and perspectives. Neuropsychiatric Electrophysiology. 2015;1:10. doi: 10.1186/s40810-015-0012-x.

\section{A73}

Changes of CNS- and ANS arousal levels following successful antidepressant treatment with ketamine: a case series Torsten Meyer, Annette Brühl, Sebastian Olbrich

Department for Psychiatry, Psychosomatics and Psychotherapy, University of Zurich, 8032, Zurich, Switzerland

Correspondence: Torsten Meyer (torsten.meyer@puk.zh.ch)

Neuropsychiatric Electrophysiology 2016, 2(Suppl 1):A73

\section{Introduction}

Ketamine has been established as an alternative in the treatment of therapy-resistant major depressive disorder (MDD). Although response rates are reportedly high with up to $60-70 \%$, until now no biomarkers that could predict treatment response exist. As a first step, this case series aimed at identifying electrophysiological markers of arousal that reflect alterations of ongoing neuronal activity after treatment with ketamine.

Methods

Two patients (one 65-year-old female, one 78-year-old male) with therapy-resistant depression ( $>$ two treatment -approaches with SSRIs, SNRIs or TCAs) were treated with ketamine infusion four times respectively six times during three weeks. Resting state electroencephalogram (EEG) and electrocardiogram (ECG) were recorded at baseline and after treatment with four/six time ketamine infusion. Central nervous system (CNS) arousal was assessed using Vigilance Algorithm Leipzig (VIGALL). Autonomous nervous system (ANS) function was quantified using heart rate and heart rate variability measures (HRV). Changes of depressive symptoms were assessed using Hamilton Depression Rating Scale (HDRS).

Results

Both patients showed a marked decrease of depressive symptoms with a drop from 28 HDRS to 9 HDRS after four ketamine infusions and from 20 HDRS to 6 HDRS after six infusions respectively. In parallel, both patients showed a decrease of CNS arousal levels as assessed by VIGALL with increased amounts of low vigilance stages and decreased EEGalpha peak frequencies after therapy in comparison to baseline EEG recording. Further, both patients revealed a lowered ANS arousal level as assessed by a reduction of heart rate $>24 \mathrm{~h}$ after the last ketamine infusion in comparison to pretreatment condition.

Discussion

Following the arousal framework in MDD with a suggested high EEGvigilance level in depression, the found decrease of CNS-arousal could be interpreted as a consequence of the anesthetic, i.e. vigilance decreasing effect of ketamine. In contrast, the decrease of heart rate remains elusive in the light of an initial increase of sympathetic function following infusion of ketamine. However, decrease of CNS- and ANS arousal level could lead to less pronounced MDD related behavioral aspects such as withdrawal and sleep disturbances. The predictive value of the EEG in ketamine treatment should be in the focus of further prospective randomized studies.

Consent to publish

Written informed consent has been obtained by all patients prior to publication.

\section{Competing interests}

The authors report no competing interests.

\section{A74}

ECoG spectral analysis of the Interaction between caffeine and nicotine

Haitham S Mohammed', Iman M Mourad ${ }^{2}$, Neveen A Noor ${ }^{2}$, Heba S Aboul Ezz ${ }^{2}$ and Yasser A Khadrawy ${ }^{3}$

${ }^{1}$ Biophysics Department, Faculty of Science, Cairo University, Giza, Egypt;

${ }^{2} Z$ oology Department, Faculty of Science, Cairo University, Giza, Egypt;

${ }^{3}$ Medical Physiology Department, Medical Division, National Research

Center, Giza, Egypt

Correspondence: Haitham S Mohammed (Haitham@sci.cu.edu.eg) Neuropsychiatric Electrophysiology 2016, 2(Suppl 1):A74

Caffeine and nicotine are the most consumed psychostimulants worldwide. Although the electrophysiological effects of each drug alone were studied extensively, the literature on the effects of their combined treatments on brain electrical activity is scarce. The present study aims to investigate the effects of the intraperitoneal injection of caffeine followed by the subcutaneous injection of nicotine after $1 \mathrm{~h}$ on electrical activity recorded from the cortex of rats $(E C O G)$. It was found that the successive injection of caffeine and nicotine resulted in a significant increase in the power of delta frequency band but a significant decrease in the power of theta, beta- 1 and beta- 2 . It was suggested that the caffeine and nicotine interaction could have an adverse effect by altering the cortical electrical activity that may indicate impair in memory encoding.

\section{A75}

Dishabituation of central nervous system to tonic pain following chiropractic care - a standardized low resolution brain electromagnetic tomography (sLORETA) based study Muhammad Samran Navid ${ }^{1,2,3}$, Dina Lelic ${ }^{1}$, Imran Khan Niazi, ${ }^{3,4}$, Kelly Holt $^{3}$, Esben Bolvig Mark', Asbjørn Mohr Drewes ${ }^{1,2}$, Heidi Haavik ${ }^{3}$

${ }^{1}$ Mech-Sense, Department of Gastroenterology and Hepatology, Aalborg University Hospital, 9000 Aalborg, Denmark; ${ }^{2}$ Department of Clinical Medicine, Aalborg University, 9100 Aalborg, Denmark; ${ }^{3}$ Centre for Chiropractic Research, New Zealand College of Chiropractic, Auckland 1060, New Zealand; ${ }^{4}$ Faculty of Health \& Environmental Sciences, Health \& Rehabilitation Research Institute, AUT University, Auckland 1010, New Zealand; ${ }^{5}$ Centre for Sensory-Motor Interactions (SMI), Department of Health Science and Technology, Aalborg University, 9100 Aalborg, Denmark

Correspondence: Muhammad Samran Navid (m.navid@rn.dk) Neuropsychiatric Electrophysiology 2016, 2(Suppl 1):A75

It has been demonstrated that after chiropractic spinal manipulation neural plastic changes occur in different areas of the brain. Different methods have been utilized to assess these changes, but the majority of the measurements to find the involved brain areas have been indirect. The objective of this study was to determine the changes in brain 
activity during tonic pain after single session of chiropractic care in a sub-clinical pain population by using source localization of the EEG.

Fifteen healthy volunteers (10 males, $32.1 \pm 7.2$ years) participated in two experimental sessions on separate days; chiropractic or control (sham) session in random order. The EEG was recorded continuously using a 61-channel system before and after either intervention during $72 \mathrm{~s}$ of cold pressor test at $2^{\circ} \mathrm{C}$ (left hand). The pain and unpleasantness ratings were obtained on two separate numeric scales (range: $0=$ no unpleasantness/pain to $10=$ maximum unpleasantness/pain). The EEG was divided into 9 epochs ( $8 \mathrm{~s}$ each), which were separated into four frequency bands: delta $(1-4 \mathrm{~Hz})$, theta $(4-8 \mathrm{~Hz})$, alpha $(8-12 \mathrm{~Hz})$ and beta $(12-32 \mathrm{~Hz})$. Subsequently, standardized low resolution brain electromagnetic tomography (sLORETA) was done on these frequency bands.

In the control experiment, the brain activity decreased in all frequency bands (all $p \leq 0.05$ ), whereas no change in activity was seen after the chiropractic session (all $p>0.05$ ). The decrease in activity in the control arm was specifically seen in the limbic (delta), frontal (theta) and temporal (alpha and beta) lobes The pain scores decreased in control arm $(p<0.05)$ whereas the unpleasantness scores decreased for both interventions (all $p<0.05$ ).

The decrease in brain activity in the control arm reflects central habituation which occurs due to repetitive painful stimulation. The lack of this phenomenon in the chiropractic arm could imply that the chiropractic care normalizes the central nervous system leading to central dishabituation.

\section{A76}

\section{Animal 3D brain-mapping}

Václava Piorecká á,2, Vladimír Krajča ${ }^{1,2}$ \& Tomáš Páleníček ${ }^{1,3}$

${ }^{1}$ Faculty of Biomedical Engineering, Czech Technical University in

Prague, Prague, Czech Republic; ${ }^{2}$ National Institute of Mental Health

Topolová 748, 250 67, Klecany, Czech Republic; ${ }^{3} 3$ rd Faculty of Medicine,

Charles University in Prague, Prague, Czech Republic

Correspondence: Václava Piorecká (vaclava.sedlmajerova@fbmi.cvut.cz) Neuropsychiatric Electrophysiology 2016, 2(Suppl 1):A76

\section{Introduction}

To this date there are no standardized mapping methods that display animal cortical EEG on the brain surface. Therefore, this study describes a 3D imaging method to be used for EEG mapping on the surface of the rat brain. The aim of our study was to develop a software module and a standard for statistical brain mapping. Animal EEG data recorded during behavioral activity and inactivity served as a subject for analysis and brain mapping.

\section{Methods}

In this study we measured electrical activity of the rat brain. For imaging purpose, we used 3D brain model from atlas [1] and adjusted it for our own module. We confirmed the validity of the 3D brain model by comparing the dimensions of normalized brain scans of 9 rats of the Wistar strain typically used in our laboratories. We have created a MATLAB module for brain mapping with the use of the rat brain model and a possibility to place any number of electrodes on the surface of the rat brain. The spline interpolation was used for imaging activity on surface of the brain [2] and statistical brainmapping was used to compare the two example behavioral conditions.

Results

The module was effectively used to display EEG activity on the 3D surface and to display the statistical group differences in the sample of the animal data between behavioral activity and inactivity. The module can also compare data from individual measurement with a group mean.

\section{Conclusions}

This study describes computation of splines interpolation curves that are important for the brain mapping in rats. This approach will be used for effective comparisons of brain activity of rats under various conditions and with variable number and placement of cortical electrodes.
This study was supported by the project Nr. LO1611 from the MEYS under the NPU I program, by project "National Institute of Mental Health (NIMH-CZ) (grant number ED2.1.00/03.0078 from the European Regional Development Fund), by Czech Technical University research program SGS (SGS15/229/OHK4/3 T/17), MH CZ DRO („National Institute of Mental Health - NIMH) project nr.: 00023752 and PRVOUK34.

I declare no conflict of interests.

\section{References}

1. MAJKA, Piotr, Jakub M. KOWALSKI, Natalia CHLODZINSKA a Daniel K WÓJCIK. 3D Brain Atlas Reconstructor Service-Online Repository of Three-Dimensional Models of Brain Structures. Neuroinformatics [online]. 2013, 11(4), 507-518 [cit. 2016-08-24]. DOI: 10.1007/s12021-0139199-9. ISSN 15392791. Online: http://link.springer.com/10.1007/ S12021-013-9199-9

2. PERRIN, F., J. PERNIER, O. BERTRAND a J.F. ECHALLIER. Spherical splines for scalp potential and current density mapping. Electroencephalography and Clinical Neurophysiology. 1989, 72(2), 184-187. DOI: 10.1016/00134694(89)90180-6. ISSN 00134694. Online: http://linkinghub.elsevier.com/ retrieve/pii/0013469489901806

A77

Effects of $\gamma$-aminobutyric acid-modulating drugs on resting state brain oscillations and executive function in healthy volunteers: a pilot study

Sara de la Salle ${ }^{1}$, Joelle Choueiry ${ }^{2}$, Danielle Impey ${ }^{1}$, Dylan Smith ${ }^{2}$, Robert Aidelbaum ${ }^{3}$, Ashley Baddeley ${ }^{4}$, Molly Hyde ${ }^{1}$, Brittany Duncan ${ }^{3}$, Justin

Piché ${ }^{\prime}$, Noreen Rahmani ${ }^{1}$, Vadim llivitsky ${ }^{5}$, Verner Knott ${ }^{1-5}$

${ }^{1}$ School of Psychology, University of Ottawa, Ottawa, Ontario, K1Z 7 K4, Canada; ${ }^{2}$ Cellular and Molecular Medicine, University of Ottawa, Ottawa, Ontario, K1N 6 N5, Canada; ${ }^{3}$ Department of Psychology, Carleton University, Ottawa, Ontario, K1S 5B6, Canada; ${ }^{4}$ Institute of Mental Health Research, Ottawa, Ontario, K1Z 7 K4, Canada; ${ }^{5}$ Department of Psychiatry, University of Ottawa, Ottawa, ON, K1N 6 N5, Canada.

Correspondence: Sara de la Salle (sara.delasalle@theroyal.ca)

Neuropsychiatric Electrophysiology 2016, 2(Suppl 1):A77

\section{Background}

Recent findings have suggested a relationship between abnormal $\gamma$ aminobutyric acid (GABA) function, disordered neuronal oscillations, and impaired executive function in schizophrenia. Additionally, there has been an increasing amount of interest in the therapeutic potential of these drugs in the treatment of this disorder. However, the neural oscillations that underlie the effects of GABA-modulating drugs on cognitive functioning require further work.

\section{Objective}

In an attempt to begin identifying which receptor subtypes may alter the neural oscillations underlying executive function via selective agonist actions, the study examined the effects of: a) a benzodiazepine drug with broad spectrum agonist actions at all GABA receptors containing the $a 1, a 2, a 3$, and $a 5$ subunits, and the $\gamma$ subunit (in addition to the obligatory $\beta$ subunit) and b) a drug with agonist actions at $G A B A_{B}$ receptors. The objective of this pilot study was to examine the effects of single doses of these GABA enhancing drugs on resting state brain oscillations and executive function in healthy volunteers stratified by executive function performance.

Method

30 participants were assessed in a randomized, double-blind, placebocontrolled design. Three minutes of eyes closed resting state brain oscillations were measured from 8 electrode sites in response to an acute administration of lorazepam (Ativan ${ }^{\circledast} ; 1.0 \mathrm{mg}$ ), a GABA $\mathrm{A}_{\mathrm{A}}$ receptor positive allosteric modulator, and baclofen (Lioresal ${ }^{\oplus} ; 10 \mathrm{mg}$ ), a GABA $\mathrm{GA}_{B}$ receptor agonist. Executive function was assessed using the Groton Maze Learning Task (GMLT) of the CogState Schizophrenia Battery.

Results

Spectral analysis revealed overall reductions in alpha and theta oscillations with the lorazepam treatment. Follow-up analyses indicated that these reductions were in the better performing participants. Correlational analyses revealed that greater lorazepam-induced reductions in alpha and theta oscillations were associated with greater 
lorazepam-induced cognitive impairment. Reduced theta at placebo was also associated with worse performance. Additionally, smaller theta activity at placebo was associated with greater lorazepaminduced cognitive impairment.

Conclusion

The results suggest that $\mathrm{GABA}_{\mathrm{A}}$-modulated alpha and theta oscillations are involved in the neural underpinnings of executive processing.

\section{A78}

EEG machine learning for enhanced monitoring of Alzheimer's disease and cholinergic modulation

Sonja Simpraga', Ricardo Alvarez-Jimenez², Huibert D. Mansvelder', Joop MA van Gerven ${ }^{2}$, Geert Jan Groeneveld ${ }^{2,3}$, Simon-Shlomo Poil ${ }^{1,4^{*}}$, Klaus Linkenkaer-Hansen ${ }^{*}$

'Dept. Integrative Neurophysiology, CNCR, Neuroscience Campus Amsterdam, Vrije Universiteit Amsterdam, Amsterdam, Netherlands; ${ }^{2}$ Dept. Clinical Pharmacology, Centre for Human Drug Research, Leiden, Netherlands; ${ }^{3}$ Dept. Neurology, VU University Medical Center, Amsterdam, Netherlands; ${ }^{4}$ NBT Analytics BV, Amsterdam, Netherlands Neuropsychiatric Electrophysiology 2016, 2(Suppl 1):A78

${ }^{*}$ These authors contributed equally

Scopolamine is a muscarinic acetylcholine receptor antagonist $(\mathrm{mAChR})$ that induces cognitive impairments resembling those observed in Alzheimer's disease (AD) and schizophrenia. It is used in drug development to demonstrate the reversal of the temporary scopolamine-induced cognitive deficits by a cognition enhancing compound. However, there is an urgent need for biomarkers that monitor therapeutic response; current biomarkers lack the desired accuracy, because of the large variability in healthy subjects and the often subtle disease-related changes. In EEG, pathophysiology is often expressed in multiple ways. Here we show that an integrative approach in which any biomarker that carries complementary information about a disease or therapeutic intervention can result in an accurate diagnostic index for better decision making in clinical trials.

Recently, we showed that EEG biomarker integration improves the prediction of conversion from mild cognitive impairment to Alzheimer's disease (AD) compared with a single-biomarker based prediction [1]. The integrative biomarker index can be used for stratification of patients at recruitment in clinical studies and for documenting and quantifying effects of intervention. Here, we provide additional proof-of-concept that EEG-based prediction can be improved with the integrative biomarker approach in clinical trials where a drug is tested in a scopolamine challenge model in healthy subjects.

For this purpose, we have developed an integrative EEGbiomarker index (mAChR index) that is optimally sensitive to the CNS effects of scopolamine, to objectively determine whether reversal of scopolamine effects by a cholinergic compound is successful. The mAChR index yielded higher classification performance than any individual EEG biomarker with accuracy, sensitivity, specificity and precision ranging from 88-92 \%. This significantly outperforms the single-best EEG biomarker (relative delta power). Validation on an independent dataset indicated the robustness of the index. To support the validity of scopolamine as a model for $A D$ pathophysiology, we show that the $m A C h R$ index discriminates healthy elderly from patients with $A D$.

We address this by using novel features of the Neurophysiological Biomarker Toolbox (http://www.nbtwiki.net/), which employ datamining algorithms to combine the information from multiple biomarkers. Our results demonstrate that integrating information from multiple EEG biomarkers can enhance the accuracy of identifying disease or drug intervention, which should be of interest to a wide range of clinical trials.

\section{References}

1. Poil SS, de Haan W, van der Flier WM, Mansvelder HD, Scheltens $P$, Linkenkaer-Hansen K. Integrative EEG biomarkers predict progression to Alzheimer's disease at the MCl stage. Front Aging Neurosci. 2013; 5:58.

\section{A79}

Midfrontal theta dynamics reflect the ability to overcome motivational biases in decision making Jennifer C Swart ${ }^{1}$, Jessica I Määttä ${ }^{1}$, Roshan Cools ${ }^{1,2}$, \& Hanneke EM den Ouden ${ }^{1}$

${ }^{1}$ Donders Institute for Brain, Cognition and Behavior, Radboud University, Nijmegen, The Netherlands; ${ }^{2}$ Dept psychiatry, Radboud

University Medical Centre, Nijmegen, The Netherlands

Correspondence: Jennifer C Swart (j.swart@donders.ru.nl)

Neuropsychiatric Electrophysiology 2016, 2(Suppl 1):A79

Our motivations influence our actions in predictable ways. The promise of a reward promotes behavioural activation, while the threat of a punishment context promotes inhibition. However, these motivational biases can at times be at odds with our goals. At such times, we need to be able to suppress them, which has been suggested to be implemented by the midfrontal cortex. We developed a novel paradigm and computational models of behavior to disentangle the impact of such motivational response biases, from the impact of learning from reward and punishment outcomes. Participants ( $N=$ 34) completed this task while recording surface EEG. As expected, cue valence strongly biased action. Midfrontal theta-band oscillatory activity was increased in those trials, where the motivational response bias conflicted with the required response, particularly when subjects successfully suppressed the motivational bias. We will present further analyses to dissociate the role of midfrontal cortex in learning from reward and punishment outcomes. This work will allow us to characterize how motivations drive biases in both choice and learning, and how we may learn to suppress these when they are at odds with our instrumental goals. This work has relevant implications for a range of psychiatric disorders associated with a maladaptive reliance on impulsive, motivationdriven responding including addiction, impulse control and ADHD.

A80

Neurophysiological substrates of memory processes: assessment of glutamatergic and cholinergic modulation of sharp wave ripples in rats

K Tahon', DA Jackson', S Jacob ${ }^{1,2}$, WH Drinkenburg ${ }^{1}$

'Department of Neuroscience Discovery, Janssen Research \&

Development, a Division of Janssen Pharmaceutica NV, B-2340, Beerse,

Belgium; ' 2 Laboratory of Biological Psychology, University of Leuven, B-

3000, Leuven, Belgium

Neuropsychiatric Electrophysiology 2016, 2(Suppl 1):A80

Sharp wave ripples (SPW-Rs) represent the most synchronous population patterns observed in the mammalian brain and are considered a cognitive biomarker for episodic memory and planning. SPW-Rs occur during several off-line states of the brain including non-REM sleep; are modulated by many neurotransmitter systems; and affect both cortical and subcortical structures by their excitatory output. Selective disruption of SPW-Rs impairs memory formation and pathological SPW-Rs have been observed in rodent models for neurodegenerative diseases. Quantification of these synchronous population patterns associated with memory processes is instrumental for a better comprehension of neurodegenerative diseases as well as for assessing the efficacy of novel pharmacological treatments. The purpose of this study was twofold: first, to develop and val- 
idate a novel computer-automated touchscreen-based spatial search task assessing either working memory or memory consolidation in Long-Evans rats; second, to quantify SPW-Rs' activity in this spatial search task during working memory or memory consolidation combined with pharmacological glutamatergic and cholinergic modulation. For the working memory component of the task, rats had to find a hidden location on the touchscreen with either a short (2 s) or long (10 s) delay between 10 consecutive trials with each delay having 4 different locations presented within one session. During these delays; hippocampal SPW-Rs from the CA1 stratum pyramidal cell layer were measured following each completed trial, using implanted 4-shank silicon electrodes. Here, SPW-Rs were measured when the rat was moving at speeds of less than $4 \mathrm{~cm} / \mathrm{s}$, by use of video monitoring to ensure events analyzed were associated with quiescent periods only. Results indicate that Scopolamine $0.1 \mathrm{mg} / \mathrm{kg}$ but not $0.05 \mathrm{mg} / \mathrm{kg}$ decreased performance for the long but not for the short delay. For the memory consolidation component of the task, rats received 1-day or 4-day acquisition session/s of a single hidden location with variable encoding strength using few $(<10)$ or many $(>40)$ trials per session. Memory consolidation of the location was measured $24 \mathrm{~h}$ after acquisition by the use of a probe trial. SPW-Rs were measured when the rats were asleep both before and after the acquisition session. A differential effect on encoding versus consolidation was addressed using pharmacological manipulation of glutamatergic and cholinergic systems. This study reinforces the strength of combining neurophysiological and cognitive behavioral assessment to further understand memory processes and effects of pharmacological treatments thereon.

\section{A81}

Glutamatergic deficit and negative symptoms: new evidence from

the ketamine model of schizophrenia

Stephanie Thiebes ${ }^{1}$, Gregor Leicht ${ }^{1}$, Stjepan Curic ${ }^{1}$, Saskia

Steinmann', Nenad Polomac', Iris Eichler ${ }^{2}$, Lars Eichler ${ }^{2}$, Christian Zöllner $^{2}$, Jürgen Gallinat ${ }^{3}$, lleana Hanganu-Opatz ${ }^{4}$, Christoph Mulert

${ }^{1}$ Psychiatry Neuroimaging Branch, Department of Psychiatry and Psychotherapy, University Medical Center Hamburg-Eppendorf, 20251Hamburg, Germany; ${ }^{2}$ Department of Anesthesiology, University Medical Center Hamburg-Eppendorf, 20251 Hamburg, Germany; ${ }^{3}$ Department of Psychiatry and Psychotherapy, University Medical Center Hamburg-Eppendorf, 20251 Hamburg, Germany:

${ }^{4}$ Developmental Neurophysiology, Institute of Neuroanatomy, University Medical Center Hamburg-Eppendorf, 20251 Hamburg, Germany

Correspondence: Stephanie Thiebes (s.thiebes@uke.de)

Neuropsychiatric Electrophysiology 2016, 2(Suppl 1):A81

Targeting the N-methyl-D-aspartate-receptor (NMDAR) is a major approach for treating negative symptoms of schizophrenia. The ketamine model of schizophrenia has the advantage of comprehensively producing schizophrenia like symptoms such as positive, cognitive and negative symptoms in healthy volunteers. The amplitude of the Mismatch Negativity (MMN), a neurophysiological parameter related to infrequent stimuli, is known to be significantly reduced in schizophrenic patients but also in healthy controls receiving ketamine [1, 2]. Accordingly, it was the aim of the present study to investigate whether changes of MMN during ketamine administration are related to the emergence of negative symptoms in healthy subjects.

Therefore, we examined the impact of ketamine on MMN amplitudes and its sources (sources localization approach: low resolution electromagnetic tomography (LORETA)) by means of 64-channel electroencephalography (EEG) recording during performance of an auditory MMN paradigm and assessed the psychopathological status using the Altered State of Consciousness (5D-ASC) Rating Scale and the Positive and Negative Syndrome Scale (PANSS). Twenty-four male, healthy volunteers were measured with pharmacological EEG using a single-blind, randomized, placebo-controlled crossover design.
We identified significant changes of the MMN response, to both duration and frequency deviants, under ketamine condition as well as a significant increase in all PANSS scores. Reductions of MMN amplitudes were significantly correlated with more pronounced negative symptoms, assessed by the PANSS.

Accordingly, the MMN might represent a biomarker for negative symptoms in schizophrenia related to an insufficient NMDAR system and could be used to identify schizophrenia patients with negative symptoms due to NMDAR dysfunction and thus to determine a maximal benefit of drugs modulating neurotransmission at the NMDAR.

Competing interests

The authors declare that they have no competing interests.

References

1. Shelley AM, Ward PB, Catts SV, Michie PT, Andrews S, McConaghy N. Mismatch negativity: an index of a preattentive processing deficit in schizophrenia. Biol Psychiatry. 1991; 30(10):1059-1062.

2. Umbricht $D$, Koller R, Vollenweider FX, Schmid L. Mismatch negativity predicts psychotic experiences induced by NMDA receptor antagonist in healthy volunteers. Biol Psychiatry. 2002; 51(5):400-406.

A82

Modulation of the serotonin system in an animal model of psilocin-induced psychosis - time course of quantitative eeg changes

Filip Tylš̌̌, , Čestmír Vejmola ${ }^{1,2}$, Lukáš Kadeřábek ${ }^{1,2}$, Václava Piorecká ${ }^{1,3}$, Vlastimil Koudelka', Tomášs Novák ${ }^{1,2}$, Tomáś Páleníček ${ }^{1,2}$

${ }^{1}$ National Institute of Mental Health Topolová 748, 250 67, Klecany, Czech Republic; ${ }^{3}$ rd Faculty of Medicine, Charles University in Prague, Prague, Czech Republic; ${ }^{3}$ Faculty of Biomedical Engineering, Czech

Technical University in Prague, Prague, Czech Republic

Correspondence: Filip Tylš (filip.tyls@nudz.cz)

Neuropsychiatric Electrophysiology 2016, 2(Suppl 1):A82

\section{Introduction}

The serotonergic hallucinogen psilocybin and its active metabolite psilocin nowadays receive a lot of attention in the scientific community as a research tool for modeling psychosis. First experiments assessing brain activity after psilocybin administration in humans using PET and fMRI found contradictory results $[1,2]$. More recently, a study directly measuring neuronal activity using MEG confirmed massive inhibition of brain activity [3]. The aim of our animal study was to assess psilocin-induced changes in quantitative EEG (QEEG) in rats in order to explore the role of different serotonergic receptors in psilocin action.

Methods

The substances used were: psilocin ( $4 \mathrm{mg} / \mathrm{kg}$ s.c.), $5 \mathrm{HT} 1 \mathrm{~A}$ antagonist WAY 100635 maleate (1 mg/kg s.c.), 5HT2A antagonist MDL-100907 tartarate $(0.5 \mathrm{mg} / \mathrm{kg}$ s.c.), $5 \mathrm{HT} 2 \mathrm{C}$ antagonist SB-242084 (1 mg/kg s.c.), haloperidol $0.1 \mathrm{mg} / \mathrm{kg}$ s.c. and clozapine $5 \mathrm{mg} / \mathrm{kg}$ i.p. For EEG experiments, rats were stereotactically implanted with 12 active electrodes onto the surface of the cortex under isoflurane anesthesia. EEG was recorded in freely moving rats after one-week recovery from surgery. EEG power spectra (local synchronization) and coherence (long projections) were subsequently analyzed comparing the drugs' effect in time (20-30, 50-60 and 80-90 min post administration) to the baseline record. To avoid moving artifacts and effects of behavior on EEG, only EEG traces corresponding to behavioral inactivity were included in the analysis.

Results

Psilocin generally decreased both EEG absolute spectral power and EEG coherences. The changes in spectral power induced by psilocin were normalized partially by all substances used, mainly in the lower frequency bands. However, only 5HT1A and 5HT2A antagonists partially normalized the psilocin-induced decrease of EEG coherences. The specific QEEG pattern of each substance and the temporal dynamics of QEEG changes will be presented. 


\section{Conclusions}

Psilocin-induced changes in QEEG in rats are very similar to our recent human data with psilocybin and are in accordance with the concept of psychosis as a disconnection syndrome. All the specific $5 \mathrm{HT}$ antagonists and both antipsychotic drugs specifically affected the EEG spectral power induced by psilocin. Surprisingly, only $5 \mathrm{HT} 1 \mathrm{~A}$ and $5 \mathrm{HT} 2 \mathrm{~A}$ antagonists were able to partially reverse psilocininduced disconnection. These results indicate that $5 \mathrm{HT} 1 \mathrm{~A}$ and $5 \mathrm{HT} 2 \mathrm{~A}$ receptors might be involved in the increase of entropic brain activity during psychedelic state as well as acute psychosis.

This study was supported by the grant IGA MHCR NT/13897, by Charles University research program PRVOUK P34, by project "National Institute of Mental Health (NIMH-CZ)", grant number ED2.1.00/03.0078, and by the European Regional Development Fund. I declare no conflict of interests.

\section{References}

1. Vollenweider F.X., Leenders K.L, Scharfetter C., Maquire P., Stadelmann O., Angst J., 1997. Positron emission tomography and fluorodeoxyglucose studies of metabolic hyperfrontality and psychopathology in the psilocybin model of psychosis. Neuropsychopharmacol, 16(5):357-372.

2. Carhart-Harris R.L., Erritzoe D., Williams T., Stone J.M., Reed L.J., Colasanti A., Tyacke R.J., Leech R., Malizia A.L., Murphy K., Hobden P., Evans J., Feilding A., Wise R.G., Nutt D.J., 2012. Neural correlates of the psychedelic state as determined by fMRI studies with psilocybin. Proc Natl Acad Sci USA, 109(6):2138-2143

3. Muthukumaraswamy S.D., Carhart-Harris R.L., Moran R.J., Brookes M.J., Williams T.M., Errtizoe D., Sessa B., Papadopoulos A., Bolstridge M., Singh K.D., Feilding A., Friston K.J., Nutt D.J., 2013. Broadband cortical desynchronization underlies the human psychedelic state. J Neurosci, 33(38):15171-15183.

\section{A83}

Novel methods assessing electrophysiological alterations by 5 HT2C receptor agonist CP-809,101 in sleep EEG and power spectral activity

Peter Veselcic, Jennifer Mollon, Emmanouil Spanakis, Maria Vasileva, Karsten Wicke

AbbVie Deutschland GmbH \& Co.KG, Knollstrasse, 67061 Ludwigshafen, Germany

Neuropsychiatric Electrophysiology 2016, 2(Suppl 1):A83

The serotonergic $5-\mathrm{HT} 2 \mathrm{C}$ receptor is a key contributor to a variety of medical conditions including psychiatric and neurological diseases. The development of therapeutic approaches at this receptor, with both, agonists and antagonists continues to be in focus [1]. Using a novel wireless EEG device (Neural Activity Tracker-1) and a novel in-house developed statistical algorithm we investigated electrophysiological changes in sleep structure and EEG power spectral distribution caused by the highly selective $5-\mathrm{HT} 2 \mathrm{C}$ receptor agonist CP-809,101.

In two independent studies, male Fischer rats with chronically implanted supracortical EEG-electrodes were treated with $10 \mathrm{mg} / \mathrm{kg}$ of CP-809,101. In the 1st study, sleep structure changes in terms of total sleep time, percent of time spent in different vigilance states, the number of rapid eye movement (REM) episodes, and latency to first sleep and REM episode were analyzed. Treatment with CP-809,101 led to attenuation of time spent in mild, deep, and REM sleep. It increased time spent in wake state and latency to first sleep and first REM episode.

The 2 nd study investigated power spectral distribution changes. A refined statistical method of baseline-adjusted power spectral changes revealed an attenuation of delta and theta band by CP-809,101 in comparison to vehicle recordings while maintaining the delta/theta ratio. Our results clearly demonstrate that acute treatment with CP809,101 changes both sleep architecture and power spectral parameters in Fischer rats.
5-HT2C agonists have been suggested to exhibit antidepressant-like profile that fits to the sleep changes observed in our study. Further, 5-HT2C agonists have been reported to inhibit theta oscillation, desynchronizing the EEG and leading to shifts to lower frequencies $[2,3]$. Yet, despite the inhibition of theta oscillation and desynchronization of the EEG by $\mathrm{CP}-809,101$, the ratio between delta/theta revealed no changes underlying the wake-promoting effects of CP-809,101.

Disclosures

All authors are employees of AbbVie. The design, study conduct, and financial support for this research was provided by AbbVie. AbbVie participated in the interpretation of data, review, and approval of the publication.

\section{References}

1. Cunningham, K. A., Fox, R. G., Anastasio, N. C., Bubar, M. J., Stutz, S. J., Moeller, F. G., ... \& Rosenzweig-Lipson, S. (2011). Selective serotonin 5-HT $2 \mathrm{C}$ receptor activation suppresses the reinforcing efficacy of cocaine and sucrose but differentially affects the incentive-salience value of cocainevs. sucrose-associated cues. Neuropharmacology, 61(3), 513-523.

2. Hajós, M., Hoffmann, W. E., \& Weaver, R. J. (2003). Regulation of septohippocampal activity by 5 -hydroxytryptamine $2 \mathrm{C}$ receptors. Journal of Pharmacology and Experimental Therapeutics, 306(2), 605-615.

3. Sörman, E., Wang, D., Hajos, M., \& Kocsis, B. (2011). Control of hippocampal theta rhythm by serotonin: role of $5-\mathrm{HT} 2 \mathrm{c}$ receptors. Neuropharmacology, 61(3), 489-494.

\section{A84}

\section{P300 in pharmacological models of psychosis}

Michaela Viktorinova $a^{1,2}$, Filip Tyls ${ }^{1,2}$, Tomas Novak ${ }^{1,2}$, Anna Bravermanova ${ }^{1,2}$, Vlastimil Koudelka ${ }^{1,2}$, Martin Brunovsky ${ }^{1,2}$ \& Tomas Palenicek ${ }^{1,2}$

${ }^{1}$ National Institute of Mental Health Topolová 748, 250 67, Klecany, Czech Republic; ${ }^{2} 3^{\text {rd }}$ Faculty of Medicine, Charles University in Prague, Prague, Czech Republic

Correspondence: Michaela Viktorinova (michaela.viktorinova@nudz.cz) Neuropsychiatric Electrophysiology 2016, 2(Suppl 1):A84

\section{Background}

P300 (P3) is an index of focal attention processes and memory updating. Impaired cognition is one of hallmark features of psychotic disorders. Both psilocybin (5-HT2A agonist) and cannabinoids induce acute transient psychotic symptoms and have previously been used as putative models for psychosis. In order to investigate the extent of cognitive disruption during psilocybin and cannabis intoxication, information processing was evaluated by means of both sensory event related potentials (P2, N2) and cognitive potential P3.

\section{Methods}

Data from two separate studies are presented.

1) In a placebo-controlled design, 20 healthy adults were administered a dose of psilocybin per os $(0,26 \mathrm{mg} / \mathrm{kg})$ and placebo during 2 separate sessions.

2) In an ecologically valid model of cannabis intoxication, 34 recreational users, 32 chronic users and 30 healthy age- and gendermatched cannabis non-users were recruited. ERPs were recorded in a sound-attenuated room with each participant lying down with their eyes closed in a comfortable setting with two sitters (male and female) being present for the whole time. An oddball paradigm with 120 frequent and 30 target tones presented binaurally in a pseudo-random order was used. Data were acquired with a standard 32-channel digital EEG amplifier BrainScope (unimedis, Prague) with 20 active scalp electrodes and oculogram according to the 10/20 system.

Results

Psilocybin: A repeated-measures ANOVA on latencies and amplitudes of $\mathrm{P} 2, \mathrm{~N} 2$ and $\mathrm{P} 3$ revealed significant effect of psilocybin only on $\mathrm{P} 3$ amplitude. Further analysis showed correlations between P3 amplitude and selected variables from objective and subjective rating scales. Cannabis: While the groups did not differ in P3 latency or amplitude, ANOVA revealed a main effect of group for P2 
component with recreational users displaying smaller amplitudes than healthy controls. Furthermore, N2 latency in recreational users was shorter when compared to healthy controls.

\section{Conclusion}

In line with previous findings, psilocybin induced abnormalities in higher-order cognition. Impaired processing is likely to be related to heightened activity of the serotonergic system at the peak of psilocybin intoxication. Although P2 findings in cannabis model indicate attention difficulties, non-significant $\mathrm{P} 3$ results need to be considered as well and are discussed in light of the ecological validity of the model.

This study was supported by the grant IGA MHCR NT/13897, by Charles University research program PRVOUK P34, by project "National Institute of Mental Health (NIMH-CZ)", grant number ED2.1.00/ 03.0078, and by the European Regional Development Fund. I declare no conflict of interests.

\section{A85}

\section{Frontal alpha asymmetry in depression: fact or fiction?}

A meta-analysis

Nikita van der Vinne ${ }^{1,2}$, Madelon A Vollebregt ${ }^{2,3}$, Martijn Arns ${ }^{2,4}$

${ }^{1}$ Synaeda Psychomedical Center, Leeuwarden, The Netherlands; ${ }^{2}$ Research Institute Brainclinics, Nijmegen, The Netherlands; ${ }^{3}$ Radboud University Medical Centre, Donders Institute for Brain, Cognition and Behaviour, Department of Cognitive Neuroscience, Nijmegen, The Netherlands; ${ }^{4}$ Department of Experimental Psychology, Utrecht University, Utrecht, The Netherlands

Correspondence: Nikita van der Vinne (n.van.der.vinne@synaeda.nl) Neuropsychiatric Electrophysiology 2016, 2(Suppl 1):A85

In major depressive disorder (MDD) research, frontal alpha asymmetry (FAA) has frequently been reported as a potential discriminator between depressed and healthy individuals, although contradicting studies and non-significant results have been published [1, 2]. Locating an MDD biomarker could benefit many people, as MDD is predicted by the WHO to become the second most debilitating disease by 2020 . The aim of the current metaanalysis is to clarify the relationships between MDD and FAA further, through analyzing new research from the last decade and put it in perspective by comparing current and past findings (for example a meta-analysis [1]).

Cohen's $d$ will be calculated from the means and standard deviations for FAA measures (subtracting mean log transformed left midfrontal alpha from mean log transformed right midfrontal alpha $[\ln (\mathrm{F} 4)-\ln (\mathrm{F} 3)])$, or a similar measure. Possible covariates including age, gender, handedness, year of publication, country of residence, depression severity, medication, EEG recording length, keeping eyes either open, closed or both, EEG reference, and used alpha frequency will be explored. A study will be included if the article (1) reports on both depressed and healthy individuals, (2) provides an FAA measure involving F3 and F4, and (3) provides all data regarding above mentioned covariates (reported either directly or obtained through contact with corresponding authors).

Preliminary results of our currently ongoing meta-analysis will be presented. On the one hand, previous studies have reported relative more left-sided alpha in MDD (sometimes only for higher frequency alpha and not for every EEG montage). On the other hand, nonsignificant and even opposite results have been reported, showing no baseline FAA differences between depressed patients and controls, or finding relatively more right-sided frontal alpha. Our expectation is that there will be no difference in FAA between MDD and non-MDD groups, based on more recent studies reporting contradicting results, as well as today's largest investigated sample regarding this topic, the iSPOT-D study [2], showing non-significant results. If non-significance is indeed demonstrated, the use of FAA as a diagnostic tool can be questioned. Nevertheless, its contribution to other applications (such as treatment prediction) could be further explored.

\section{References}

1. Thibodeau R, Jorgensen R, Kim S. Depression, Anxiety, and Resting Frontal EEG Asymmetry: A Meta-Analytic Review. J Abnorm Psychol. 2006; 115;715-729.

2. Arns M, Bruder G, Hegerl U, Spooner C, Palmer D, Etkin A, Fallahpour K, Gatt J, Hirshberg L, Gordon E. EEG alpha asymmetry as a gender-specific predictor of outcome to acute treatment with different antidepressant medications in the randomized iSPOT-D study. Clin Neurophysiol. 2016; 127:509-519.

A86

Neuropharmacological profile of selected areas responsible for the inhibition of P50 wave: from the P50 wave to off-label treatment of schizophrenia

Premysl Vlcek, Barbora Kohutova, Jakub Polak, Martin Brunovsky

National Institute of Mental Health, Klecany; The Department of Applied

Brain Electrophysiology (ABEP), Klecany, Czech Republic

Neuropsychiatric Electrophysiology 2016, 2(Suppl 1):A86

Patients suffering from schizophrenia have been shown to exhibit impaired P50 ERP amplitude-reduction to the second (S2) relative to the first (S1) of identical brief auditory stimuli. This reduction is often mentioned in connection with the inability to filter redundant sensory stimuli typically manifested as inability to gate neuronal responses related to the P50 wave [1, 2]. The key neuronal structure responsible for the sensory gating process is the hippocampus. Inhibition of redundant stimuli in the hippocampus is affected via the release of glutamate from excitatory pathways, which is controlled by GABAB receptors. It is closely connected with a physiological deficit of hippocampal GABAergic interneurons, which demonstrates neuropathological changes in schizophrenia. Several drugs are able to improve sensory gating, the effect of which is explained by their ability to disinhibit GABAergic neurons in the hippocampus. The effect of setrons may be an example of such effective gabaergic interneurons disinhibition. This antagonist of 5-HT 3 receptors increased (by disinhibition of gabaergic interneurons) release of acetylcholine, which by agonism of alpha7 nicotinic receptors improved auditory gating [3]. Besides the hippocampus the prefrontal cortex is an important neuronal part of the sensory gating. Patients with a prefrontal damage fail to suppress irrelevant sensory information, which leads to increased neural noise and inability to inhibit taskirrelevant information during behavioral tasks requiring performance over a delay. Some of the P50 source analysis leads to the conclusion that while the temporal cortices are the main generator of the P50 component, the prefrontal cortex seems to be a main contributor to the process of sensory gating (P50 amplitude reduction) [4]. As in the case of the hippocampus, there are drugs that improve sensory gating by acting on the prefrontal cortex. Clonidine acts as an agonist of a $2 \mathrm{~A}$ noradrenergic receptors and has a proven restorative effect on sensory gating. Stimulation of a $2 \mathrm{~A}$ noradrenergic receptors on PFC spines by clonidine leads to strengthening of network connectivity, increase in neuronal PFC firing, and thus improves PFC regulation of sensory gating [5]. The aim of our poster is to interlink a pharmacological profile of neuronal areas that are involved in the inhibition of P50 wave with clinical treatment of schizophrenia. We believe that the neuropharmacological aspects of P50 wave offer an interesting hypothesis relating mainly to the pharmacological augmentation strategies. Some of them are suggested and explained further in our poster communication.

This work was supported by the project "National Institute of Mental Health (NIMH-CZ)", under grant number ED2.1.00/03.0078, the European Regional Development Fund, the Charles University research program PRVOUK P34, and the travel grant of the Czech neuropsychopharmacological society. 


\section{References}

1. Dalecki A, Green AE, Johnstone SJ, Croft RJ. The relevance of attention in schizophrenia P50 paired stimulus studies. Clin Neurophysiol. 2016 Jun;127(6):2448-54.

2. Vlcek P, Bob P, Raboch J. Sensory disturbances, inhibitory deficits, and the P50 wave in schizophrenia. Neuropsychiatr Dis Treat. 2014;10:1309-15.

3. Freedman R, Adler LE, Myles-Worsley M, Nagamoto HT, Miller C, Kisley M, et al. Inhibitory gating of an evoked response to repeated auditory stimuli in schizophrenic and normal subjects. Human recordings, computer simulation, and an animal model. Arch Gen Psychiatry. 1996 Dec;53(12):1114-21.

4. Knight RT, Staines WR, Swick D, Chao LL. Prefrontal cortex regulates inhibition and excitation in distributed neural networks. Acta Psychol (Amst). 1999 Apr;101(2-3):159-78.

5. Arnsten AF, Jin LE. Guanfacine for the treatment of cognitive disorders: a century of discoveries at Yale. Yale J Biol Med. 2012 Mar;85(1):45-58.

\section{A87}

Dopamine under the influence of sunlight? Transitions in solar irradiation explaining attentional performance in DRD4 7R carriers Madelon A Vollebregt ${ }^{1,2}$, Martijn Arns ${ }^{1,3}$

${ }^{1}$ Research Institute Brainclinics, Nijmegen, The Netherlands; ${ }^{2}$ Department of Cognitive Neuroscience, Donders Institute for Brain, Cognition and Behaviour, Radboud University Medical Centre, Nijmegen, The Netherlands; ${ }^{3}$ Dept. of Experimental Psychology, Utrecht University, Utrecht, The Netherlands

Correspondence: Madelon A Vollebregt (madelon@brainclinics.com) Neuropsychiatric Electrophysiology 2016, 2(Suppl 1):A87

\section{Background}

Previous research suggests that high exposure to solar irradiation has a preventive effect on the development of attention-deficit/ hyperactivity disorder (ADHD) [1]. Note that the Dopaminergic DRD4 receptor is involved in phototransduction in the retina. Interestingly, being a DRD4 7R carrier while being born in spring and summer has been demonstrated to result in a 2.8 higher likelihood of developing hyperkinetic disorder, equivalent to ADHD [2]. These findings suggested a possible gene $X$ environment interaction between the DRD4 7R allele and season of birth. The current study focused on the influence of solar irradiation exposure around birth on adult attentional performance.

Methods

We used an RDoC approach focusing on "inattention" operationalized as false negative errors, i.e. missed targets, from two cognitive tasks; the auditory oddball task and the continuous performance task. DRD4 genotype was regarded a vulnerability to develop ADHD, i.e. high inattention. We specifically aimed to test hypotheses that we generated based on previous studies. We distinguished the solar irradiation at birth-month from the difference in the solar irradiation between the month after birth and the month of birth to further understand previous results. Data of 277 healthy adult participants were extracted from the Brain Resource International Database. This database contains data from multiple laboratories (New York, Rhode Island, Nijmegen, Sydney, and Adelaide) creating variability in solar irradiation data.

Results

Results showed an interaction between DRD4 genotype and transition in solar irradiation following birth on the number of inattention errors made $(F(1,269)=6.785, p=.010)$. More specifically, a one-way ANOVA for the DRD4 7R carrier group showed a significant difference between positive and negative transition in solar irradiation
$(1,86)=8.602, p=.004, d=-0.449)$, while data from participants lacking the DRD4 7R genotype did not differ.

Conclusions

These results provide evidence that factors around birth influence adult performance and may strengthen or weaken the risk to develop attention related problems once already genetically at risk. Results also further strengthen the hypothesis that a relationship between solar irradiation and ADHD exists, possibly mediated by the dopamine DRD4 receptor.

\section{References}

1. Arns, M., van der Heijden K.B., Arnold L.E., Kenemans J.L. Geographic variation in the prevalence of attention-deficit/hyperactivity disorder: the sunny perspective. Biol psychiat. 2013 Oct 15;74(8):585-90.

2. Seeger, G., Schloss, P., Schmidt, M.H., Rüter-Jungfleisch, A., Henn, F.A. (2004). Gene-environment interaction in hyperkinetic conduct disorder (HD + CD) as indicated by season of birth variations in dopamine receptor (DRD4) gene polymorphism. Neuroscience Letters, 366, 282-286.

\section{A88}

A pilot study of sucrose-induced effect in resting alpha asymmetry

Grace Y Wang ${ }^{1}$, Lin Tiffany ${ }^{2}$, Nazimah Hamid ${ }^{2}$. Alex Sumich ${ }^{3}$

${ }^{1}$ Division of Psychology, Auckland University of Technology, Auckland,

New Zealand; ${ }^{2}$ School of Applied Sciences, Auckland University of

Technology, Auckland,New Zealand; ${ }^{3}$ Division of Psychology, School of

Social Sciences, Nottingham Trent University, Nottingham, UK

Correspondence: Grace Y Wang (gwang@aut.ac.nz)

Neuropsychiatric Electrophysiology 2016, 2(Suppl 1):A88

\section{Background and aim}

Prospective studies have linked sucrose consumption to weight gain and obesity, which are in turn associated with the development of a range of adverse health effects including diabetes and premature cardiovascular disease, both identified as leading causes of health loss in New Zealand and Europe. Recent work has also reported that there are cognitive deficits induced by elevated intake of sugars. There is the need to improve understanding of how the processes involved in the deterioration of cognitive functions and mechanisms are modulated by the sucrose. The present study was to investigate the effect of sucrose on resting EEG alpha asymmetry using EEG.

Methods

We recorded resting, spontaneous EEG from 64 scalp electrodes according to the international 10/20 system (NeuroScan, A/D rate: $1000 \mathrm{~Hz}$ ) while healthy volunteers $(\mathrm{N}=10)$ were orally administered sucrose (with $1 \%$ sweetness) and placebo solutions. The participants held these solutions in their mouth, still with eyes closed. EEG spectral indices from the left and right frontal (F3, F4), central $(\mathrm{C} 4, \mathrm{C} 3)$ and posterior regions (P4, P3) of the scalp were extracted by a continuous wavelet transform and normalised into spectral distribution in the alpha $(8-12 \mathrm{~Hz})$ band. Sucrose-induced change in alpha asymmetry was analysed using ANOVA, with conditions (sucrose, placebo), hemisphere (left, right) and regions (frontal, central and posterior) as the within-subjects factors.

Results

Our results showed that there was no significant condition* hemisphere effect. However, a medium effect size $(r=0.33)$ may indicate some effect which did not turn out to be significant due to the limited sample size. A tendency for a reduced asymmetry value (i.e. F4-F3) was observed when the group was exposed to sucrose.

Discussion

Our findings provide preliminary evidence of sucrose-induced change in brain activity. 